\title{
Development and validation of HERWIG 7 tunes from CMS underlying-event measurements
}

\author{
CMS Collaboration* \\ CERN, 1211 Geneva 23, Switzerland
}

Received: 6 November 2020 / Accepted: 3 February 2021 / Published online: 12 April 2021

(C) CERN for the benefit of the CMS collaboration 2021

\begin{abstract}
This paper presents new sets of parameters ("tunes") for the underlying-event model of the HERWIG 7 event generator. These parameters control the description of multiple-parton interactions (MPI) and colour reconnection in HERWIG 7, and are obtained from a fit to minimum-bias data collected by the CMS experiment at $\sqrt{s}=0.9,7$, and $13 \mathrm{TeV}$. The tunes are based on the NNPDF 3.1 next-to-nextto-leading-order parton distribution function (PDF) set for the parton shower, and either a leading-order or next-to-nextto-leading-order PDF set for the simulation of MPI and the beam remnants. Predictions utilizing the tunes are produced for event shape observables in electron-positron collisions, and for minimum-bias, inclusive jet, top quark pair, and $\mathrm{Z}$ and W boson events in proton-proton collisions, and are compared with data. Each of the new tunes describes the data at a reasonable level, and the tunes using a leading-order PDF for the simulation of MPI provide the best description of the data.
\end{abstract}

\section{Introduction}

In hadron-hadron collisions, the hard scattering of partons is accompanied by additional activity from multiple-parton interactions (MPI) that take place within the same collision, and by interactions between the remnants of the hadrons. To describe the underlying-event (UE) activity in a hard scattering process, and minimum-bias (MB) events, Monte Carlo (MC) event generators such as HERWIG 7 [1-3] and PYTHIA 8 [4] include a model of these additional interactions. Because these processes are soft in nature, perturbative quantum chromodynamics (QCD) cannot be used to predict them, so they must be described by a phenomenological model. The parameters of the models must be optimized to provide a reasonable description of measured observables that are sensitive to the UE and MB events. An accurate description of the UE by MC event generators, along with an understanding of the uncertainties in the description, is of particular importance

\footnotetext{
^e-mail: cms-publication-committee-chair@cern.ch
}

for precision measurements at hadron colliders, such as the extraction of the top quark mass. This paper presents new sets of parameters ("tunes") for the UE model of the HERWIG 7 event generator.

The HERWIG 7 event generator is a multipurpose event generator, which can perform matrix-element (ME) calculations beyond leading order (LO) in QCD, via the MATCHBOX module [5], matched with parton shower (PS) calculations. Both an angular-ordered and a dipole-based PS simulation are available in HERWIG 7, and the former is used in this paper. The ME calculations can also be provided by an external ME generator, such as POWHEG [6-8] or MADGRAPH5_aMC@NLO [9]. The HERWIG7 generator is built upon the development of the preceding HERWIG [10] and HERWIG++ [1] event generators. In addition to the simulation of hard scattering of partons in hadron-hadron collisions, a simulation of MPI, which is modelled by a combination of soft and hard interactions and by colour reconnection (CR) [1,11-13], is included in HERWIG 7. As shown in Ref. [13], a model of CR is required in HERWIG 7 to describe the structure of colour connections between different MPI, and to obtain a good description of the mean charged-particle transverse momentum $\left(p_{\mathrm{T}}\right)$ as a function of the charged-particle multiplicity $\left(N_{\mathrm{ch}}\right)$.

The model describing the soft interactions, and also diffractive processes, was improved in version 7.1 of HERWIG 7. This resulted in a new tune of the MPI parameters, called SoftTune, which improved the description of MB data [3,12]. In particular, the description of final-state hadronic systems separated by a large rapidity gap $[14,15]$ is notably improved because a significant contribution is expected from diffractive events. The tune SoftTune is based on the MMHT 2014 LO parton distribution function (PDF) set [16], and was derived by fitting MB data at $\sqrt{s}=0.9,7$, and $13 \mathrm{TeV}$ from the ATLAS experiment [17]. The MB data used in the tuning include the pseudorapidity $(\eta)$ and $p_{\mathrm{T}}$ distributions of charged particles for various lower bounds on $N_{\text {ch }}$, namely $N_{\text {ch }} \geq 1,2,6$, and 20 . The mean chargedparticle $p_{\mathrm{T}}$ as a function of $N_{\mathrm{ch}}$ was also included in the tun- 
ing procedure. Three models of CR are available in HERWIG 7, and SoftTune was derived with the plain colour reconnection (PCR) model implemented. The same PCR model is considered in our studies.

In this paper, we present new UE tunes for the HERWIG 7 (version 7.1.4) generator. In contrast to SoftTune, the tunes presented here are based on the NNPDF 3.1 PDF sets [18], and use the next-to-next-to-leading-order (NNLO) PDF set for the simulation of the PS, and either an LO or NNLO PDF set for the simulation of MPI and the beam remnants. This choice of PDF sets is similar to that used to obtain tunes for the PYTHIA 8 event generator in Ref. [19], where it was shown that predictions from PYTHIA 8 using LO, next-to-leadingorder (NLO), and NNLO PDFs with their associated tunes can all give a reliable description of the UE. Based on these findings and the wide use by the CMS Collaboration of the CP5 PYTHIA 8 tune, we concentrate on deriving tunes for the HERWIG 7 generator that are also based on an NNLO PDF set for the simulation of the parton shower. It is verified that using an NNLO PDF in the simulation of the PS in HERWIG 7 also provides a reliable description of $\mathrm{MB}$ data. A consistent choice of PDF in the HERWIG 7 and PYTHIA 8 generators, as well as a similar method of the MPI model tuning, provides a better comparison of predictions from these two generators.

The tunes are derived by fitting measurements from proton-proton collision data collected by the CMS experiment [20] at $\sqrt{s}=0.9,7$, and $13 \mathrm{TeV}$. The measurements used in the fitting procedure are chosen because of their sensitivity to the modelling of the UE in HERWIG 7. Uncertainties in the parameters of one of the new tunes are also derived. This quantifies the effect of the uncertainties in the fitted parameters for future analyses. To validate the performance of the new tunes, the corresponding HERWIG 7 predictions are compared with a range of $\mathrm{MB}$ data from protonproton and proton-antiproton collisions. Comparisons are also made using event shape observables from electronpositron collisions collected at the CERN LEP accelerator, which are particularly sensitive to the choice of the strong coupling $\alpha_{\mathrm{S}}$ in the description of final-state radiation. To further validate the new tunes, predictions of differential $\mathrm{t} \overline{\mathrm{t}}, \mathrm{Z}$ boson, and $\mathrm{W}$ boson cross sections are also obtained from matching ME calculations from POWHEG and MADGRAPH5_aMC@NLO with the HERWIG 7 PS description. The kinematics of the t't system are studied, along with the multiplicity of additional jets, which are sensitive to the modelling by the PS simulation, in $\overline{\mathrm{t}}, \mathrm{Z}$ boson, and $\mathrm{W}$ boson events. The modelling of the UE in Z boson events, and the substructure of jets in $\mathrm{t}_{\mathrm{t}}$ and in inclusive jet events are also investigated. Some of these comparisons are sensitive to the modelling by the ME calculations, and the purpose of those is to validate that the various predictions using the tunes do not differ from each other by a significant amount. Other comparisons are more sensitive to the modelling of the PS and MPI simulation, allowing us to test the new tunes in data other than MB data.

This paper is organized as follows. In Sect. 2, we summarize the UE model employed by HERWIG 7, and describe the model parameters considered in the tuning. The choice of PDF and the value of the strong coupling in the tunes is discussed in Sect. 3 in addition to details of the fitting procedure. The new tunes are presented in Sect. 4, and the corresponding predictions from HERWIG 7 are compared with MB data. Uncertainties in one of the derived tunes are presented in Sect. 5. Further validation of the new tunes is performed in the following sections: their predictions are compared with event shape observables from the CERN LEP in Sect. 6, and with top quark, inclusive jet, and $\mathrm{Z}$ and $\mathrm{W}$ boson production data in Sects. 7, 8, and 9, respectively. Finally, we present a summary in Sect. 10.

\section{The UE model in HERWIG 7}

The UE in HERWIG 7 is modelled by a combination of soft and hard interactions $[1,11,12]$. The parameter $p_{\perp}^{\min }$ defines the transition between the soft and hard MPI. The interactions with a pair of outgoing partons with $p_{\mathrm{T}}$ above $p_{\perp}^{\mathrm{min}}$ are treated as hard interactions, which are constructed from QCD twoto-two processes. The $p_{\perp}^{\min }$ transition threshold depends on the centre-of-mass energy of the hadron-hadron collision and is given by:

$p_{\perp}^{\min }=p_{\perp, 0}^{\min }\left(\frac{\sqrt{s}}{E_{0}}\right)^{b}$,

where $p_{\perp, 0}^{\min }$ is the value of $p_{\perp}^{\min }$ at a reference energy scale $E_{0}$, which is set to $7 \mathrm{TeV}, \sqrt{s}$ is the centre-of-mass energy of the hadron-hadron collision, and the parameter $b$ controls the energy dependence of $p_{\perp}^{\mathrm{min}}$. Decreasing the value of $p_{\perp}^{\mathrm{min}}$ increases the number of hard interactions whilst reducing the number of soft interactions, which typically increases the amount of activity in the UE.

The average number $\langle n\rangle$ of these additional hard interactions per hadron-hadron collision is given by:

$\langle n\rangle=A(d) \sigma(s)$,

where $\sigma(s)$ is the production cross section of a pair of partons with $p_{\mathrm{T}}>p_{\perp}^{\min }$ and $A(d)$ describes the overlap between the two protons at a given impact parameter $d$. The form of the overlap function is given by:

$A(d)=\frac{\mu^{2}}{96 \pi}(\mu d)^{3} K_{3}$,

where $\mu^{2}$ is the inverse proton radius squared, and $K_{3} \equiv$ $K_{3}(\mu d)$ is the modified Bessel function of the third kind. 
Table 1 Parameters considered in the tuning, and their allowed ranges in the fit

\begin{tabular}{lll}
\hline Parameter & HERwIG 7 configuration parameter & Range \\
\hline$p_{\perp, 0}^{\min }(\mathrm{GeV})$ & /Herwig/UnderlyingEvent/MPIHandler:pTmin0 & $1.0-5.0$ \\
$b$ & /Herwig/UnderlyingEvent/MPIHandler:Power & $0.1-0.5$ \\
$\mu^{2}\left(\mathrm{GeV}^{-2}\right)$ & /Herwig/UnderlyingEvent/MPIHandler:InvRadius & $0.5-2.7$ \\
$p_{\text {reco }}$ & /Herwig/Hadronization/ColourReconnector:ReconnectionProbability & $0.05-0.90$ \\
\hline
\end{tabular}

The overlap function is obtained by the convolution of the electromagnetic form factors of two protons. The number of additional hard interactions per hadron-hadron collision at a given $d$ is described by a Poissonian probability distribution with a mean given by Eq. (2), which is then integrated over the impact parameter space. Increasing $\mu^{2}$ increases the density of the partons in the hadrons, and results in a higher probability for additional hard scatterings to take place.

Additional soft interactions, which produce pairs of partons below $p_{\perp}^{\text {min }}$, are based on a model of multiperipheral particle production [12]. The number of additional soft interactions between the two hadron remnants is described in a similar way to the hard interactions above $p_{\perp}^{\min }$. In a soft interaction between the two hadron remnants, the mean number of particles produced is given by:

$\langle N\rangle=N_{0}\left(\frac{s}{1 \mathrm{TeV}^{2}}\right)^{P} \ln \frac{\left(p_{\mathrm{r} 1}+p_{\mathrm{r} 2}\right)^{2}}{m_{\mathrm{rem}}^{2}}$,

where $p_{\mathrm{r} 1}$ and $p_{\mathrm{r} 2}$ are the four-momenta of the two remnants, and $m_{\text {rem }}$ is the mass of a proton remnant, i.e. the remaining valence quarks of a proton treated as a diquark system, and is set to $0.95 \mathrm{GeV}$. The parameters $N_{0}$ and $P$ control the energy dependence of the mean number of soft particles produced. They were tuned to $\mathrm{MB}$ data, which resulted in the values $P=-0.08$ and $N_{0}=0.95$ [3]. In deriving the tune SoftTune the values of $N_{0}$ and $P$ were kept fixed at these values.

The cluster model [21] is used to model the hadronization of quarks into hadrons. After the PS calculation, gluons are split into quark-antiquark pairs, and a cluster is formed from each colour connected pair of quarks. Before hadrons are produced from the clusters, CR can modify the configuration of the clusters. With the PCR model, the quarks from two clusters can be reconfigured to form two alternative clusters. The change of the cluster configuration takes place only if the sum of the masses of the new clusters is smaller than before. If this condition is satisfied, the $\mathrm{CR}$ is accepted with a probability $p_{\text {reco }}$, which is the only parameter of the PCR model. The PCR model typically leads to clusters with smaller invariant mass compared with the clusters that would be obtained without $\mathrm{CR}$, and will typically reduce the overall activity in the UE.

\section{Tuning procedure}

We derive three tunes based on the NNPDF 3.1 PDF sets [18]. A different PDF set is chosen for each aspect of the HERWIG 7 simulation: hard scattering, parton showering, MPI, and beam remnant handling. The value of $\alpha_{\mathrm{S}}$ at a scale equal to the Z boson mass $m_{\mathrm{Z}}$ in each tune is set to $\alpha_{\mathrm{S}}\left(m_{\mathrm{Z}}\right)=0.118$ for all parts of the HERWIG 7 simulation, with a two-loop running of $\alpha_{\mathrm{S}}$.

The first tune, CH1 ("CMS HERWIG"), uses an NNLO PDF set in all aspects of simulation in HERWIG 7, where the PDF was derived with a value of $\alpha_{S}\left(m_{Z}\right)=0.118$. This is equivalent to the choice of PDF and $\alpha_{\mathrm{S}}\left(m_{\mathrm{Z}}\right)$ used in the CP5 PYTHIA 8 tune [19]. In the second tune, $\mathrm{CH} 2$, an LO PDF set that was also derived with $\alpha_{\mathrm{S}}\left(m_{\mathrm{Z}}\right)=0.118$, is used in the simulation of MPI and beam remnant handling, whereas an NNLO PDF set is used elsewhere. The final tune, $\mathrm{CH} 3$, is similar to $\mathrm{CH} 2$, but uses an LO PDF set that was derived with $\alpha_{\mathrm{S}}\left(m_{\mathrm{Z}}\right)=0.130$ for the simulation of MPI and remnant handling. The choice of an LO PDF set for the simulation of MPI and beam remnant handling, regardless of the choice

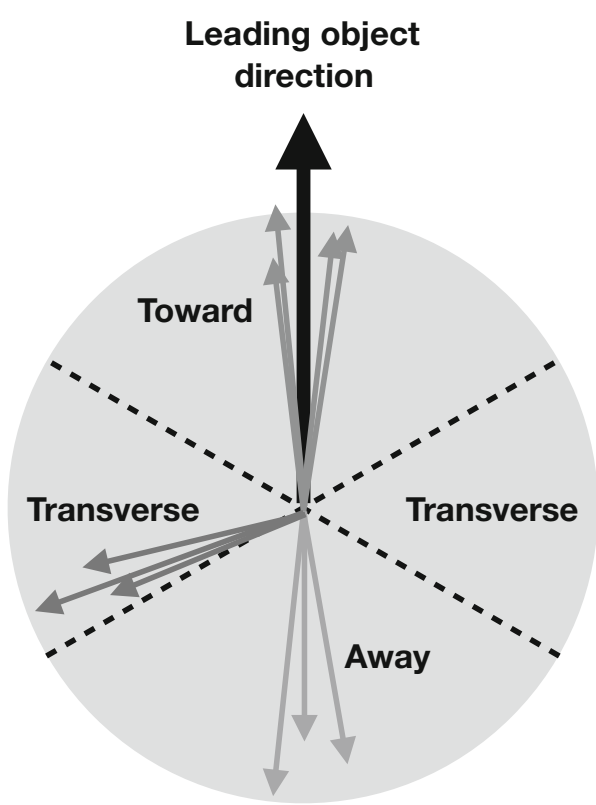

Fig. 1 Illustration of the different $\phi$ regions, with respect to the leading object in an event, used to probe the properties of the UE in measurements 
Table 2 Value of the parameters for the SoftTune [3,12], CH1, CH2, and $\mathrm{CH} 3$ tunes

\begin{tabular}{|c|c|c|c|c|c|}
\hline & & SoftTune & $\mathrm{CH} 1$ & $\mathrm{CH} 2$ & $\mathrm{CH} 3$ \\
\hline$\alpha_{\mathrm{S}}\left(m_{\mathrm{Z}}\right)$ & & 0.1262 & 0.118 & 0.118 & 0.118 \\
\hline \multirow[t]{2}{*}{ PS } & PDF set & MMHT 2014 LO & NNPDF3.1 NNLO & NNPDF 3.1 NNLO & NNPDF3.1 NNLO \\
\hline & $\alpha_{\mathrm{S}}^{\mathrm{PDF}}\left(m_{\mathrm{Z}}\right)$ & 0.135 & 0.118 & 0.118 & 0.118 \\
\hline MPI \& & PDF set & MMHT 2014 LO & NNPDF3.1 NNLO & NNPDF 3.1 LO & NNPDF 3.1 LO \\
\hline remnants & $\alpha_{\mathrm{S}}^{\mathrm{PDF}}\left(m_{\mathrm{Z}}\right)$ & 0.135 & 0.118 & 0.118 & 0.130 \\
\hline$p_{\perp, 0}^{\min }(\mathrm{GeV})$ & & 3.502 & 2.322 & 3.138 & 3.040 \\
\hline$b$ & & 0.416 & 0.157 & 0.120 & 0.136 \\
\hline$\mu^{2}\left(\mathrm{GeV}^{-2}\right)$ & & 1.402 & 1.532 & 1.174 & 1.284 \\
\hline$p_{\text {reco }}$ & & 0.5 & 0.400 & 0.479 & 0.471 \\
\hline$\chi^{2} / N_{\text {dof }}$ & & 12.8 & 6.75 & 1.54 & 1.71 \\
\hline
\end{tabular}

of PDF used in the PS and ME calculation, is motivated by ensuring that the gluon PDF is positive at the low energy scales involved, which is not necessarily the case with higherorder PDF sets. However, as was shown in Ref. [19], the gluon PDF in the NNLO NNPDF3.1 set remains positive at low energy scales, and predictions from PYTHIA 8 using LO and higher-order PDFs can both give a reliable description of $\mathrm{MB}$ data. The configurations of PDF sets in the $\mathrm{CH} 1, \mathrm{CH} 2$, and $\mathrm{CH} 3$ tunes allow us to study whether using an NNLO PDF set consistently for all aspects of the HERWIG 7 simulation, or an LO PDF set for the simulation of MPI, can both give a reliable description of $\mathrm{MB}$ data. For both of these choices the gluon PDF is positive at low energy scales.

The names of the parameters being tuned in the HERWIG 7 configuration, and their allowed ranges in the fit, are shown in Table 1. The values of $N_{0}=0.95$ and $P=-0.08$ are fixed at the values that were used in the tune SoftTune. As shown later, no further tuning of these parameters is necessary, because of the good description of measured observables obtained with these values.

The tunes are derived by fitting unfolded MB data that are available in the RIVET [22] toolkit. The proton-proton collision data used in the fit were collected by the CMS experiment at $\sqrt{s}=0.9,7$, and $13 \mathrm{TeV}$. In measurements probing the UE, charged particles in a particular event are typically categorized into different $\eta-\phi$ regions with respect to a leading object in that event, such as the highest $p_{\mathrm{T}}$ track or jet, as illustrated in Fig. 1. The difference in azimuthal $\phi$ between each charged particle and the leading object $(\Delta \phi)$ is used to assign each charged particle to a region, namely the toward $\left(|\Delta \phi| \leq 60^{\circ}\right)$, away $\left(|\Delta \phi|>120^{\circ}\right)$, and transverse regions $\left(60<|\Delta \phi| \leq 120^{\circ}\right)$. The properties of the charged particles in the transverse regions are the most sensitive to the modelling of the UE. The two transverse regions can be further divided into the transMin and transMax regions, which are the regions with the least and most charged-particle activity,

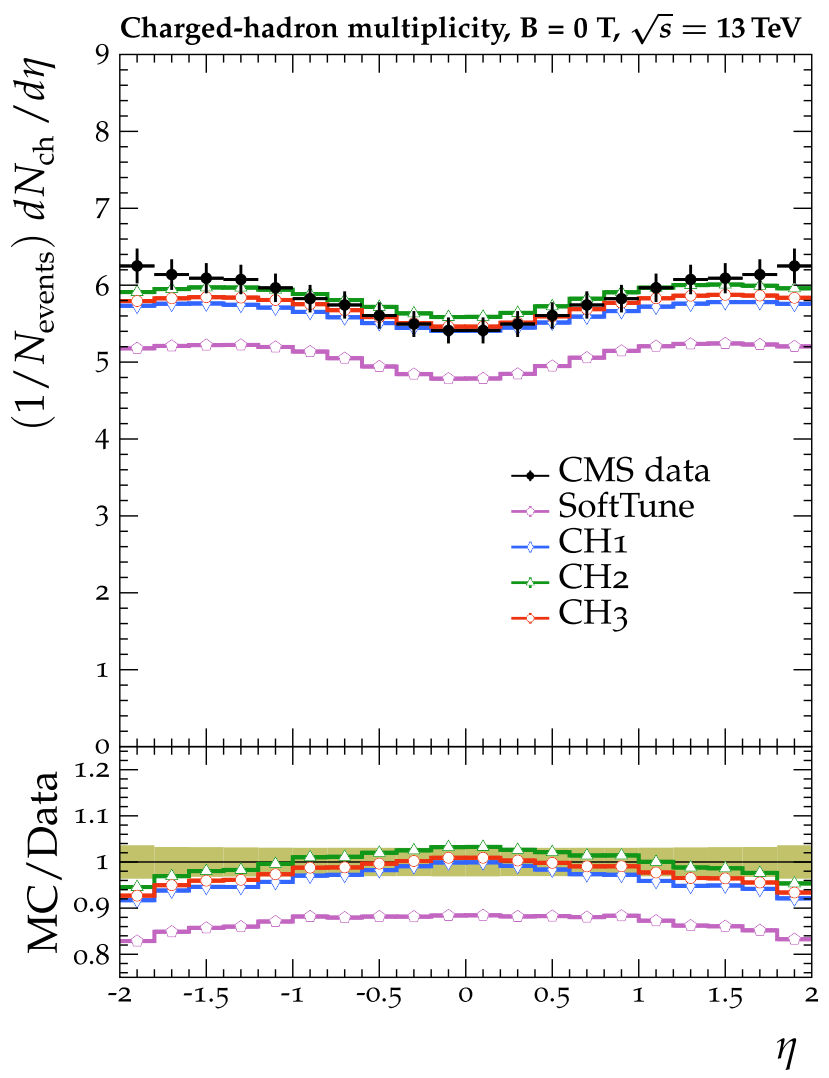

Fig. 2 The normalized $\mathrm{d} N_{\mathrm{ch}} / \mathrm{d} \eta$ of charged hadrons as a function of $\eta$ [27]. CMS MB data are compared with SoftTune and the $\mathrm{CH}$ tunes. The coloured band in the ratio plot represents the total experimental uncertainty in the data. The vertical bars on the points for the different predictions represent the statistical uncertainties

respectively. Data that have been categorized in this way are referred to as UE data in this paper.

At $\sqrt{s}=7$ and $13 \mathrm{TeV}$, the $N_{\mathrm{ch}}$ and transverse momentum sum $\left(p_{\mathrm{T}}^{\text {sum }}\right)$, with respect to the beam axis, as functions of the $p_{\mathrm{T}}$ of the leading track $\left(p_{\mathrm{T}}^{\max }\right)$ in the transMin and transMax regions are used in the fit [23,24]. At $\sqrt{s}=0.9 \mathrm{TeV}$, the observables used are the $N_{\mathrm{ch}}$ and $p_{\mathrm{T}}^{\text {sum }}$ in 

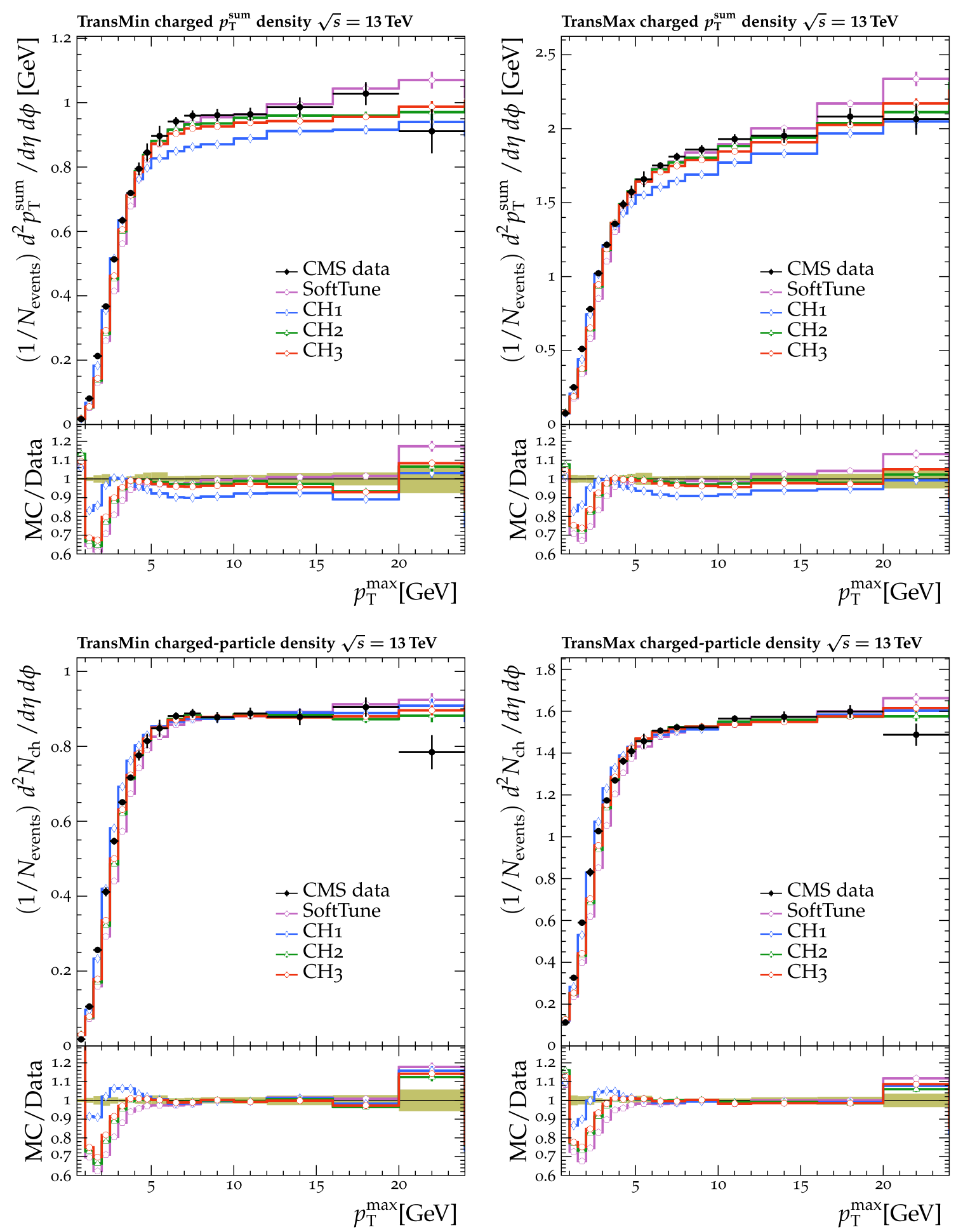

Fig. 3 The normalized $p_{\mathrm{T}}^{\text {sum }}$ (upper) and $N_{\mathrm{ch}}$ (lower) density distributions in the transMin (left) and transMax (right) regions, as a function of the $p_{\mathrm{T}}$ of the leading track, $p_{\mathrm{T}}^{\max }$ [24]. CMS MB data are compared with the predictions from HERWIG 7, with the SoftTune and $\mathrm{CH}$ tunes.

The coloured band in the ratio plot represents the total experimental uncertainty in the data. The vertical bars on the points for the different predictions represent the statistical uncertainties

the transverse region, as a function of the $p_{\mathrm{T}}$ of the leading jet $\left(p_{\mathrm{T}}^{\text {jet }}\right)[25]$. The track jets are clustered using the SISCONE algorithm [26] with a distance parameter of 0.5. The regions

$p_{\mathrm{T}}^{\max }<3 \mathrm{GeV}$ and $p_{\mathrm{T}}^{\text {jet }}<3 \mathrm{GeV}$ are not included in the fit because the parameters of diffractive processes, which dominate this region, are not considered. The charged-hadron mul- 

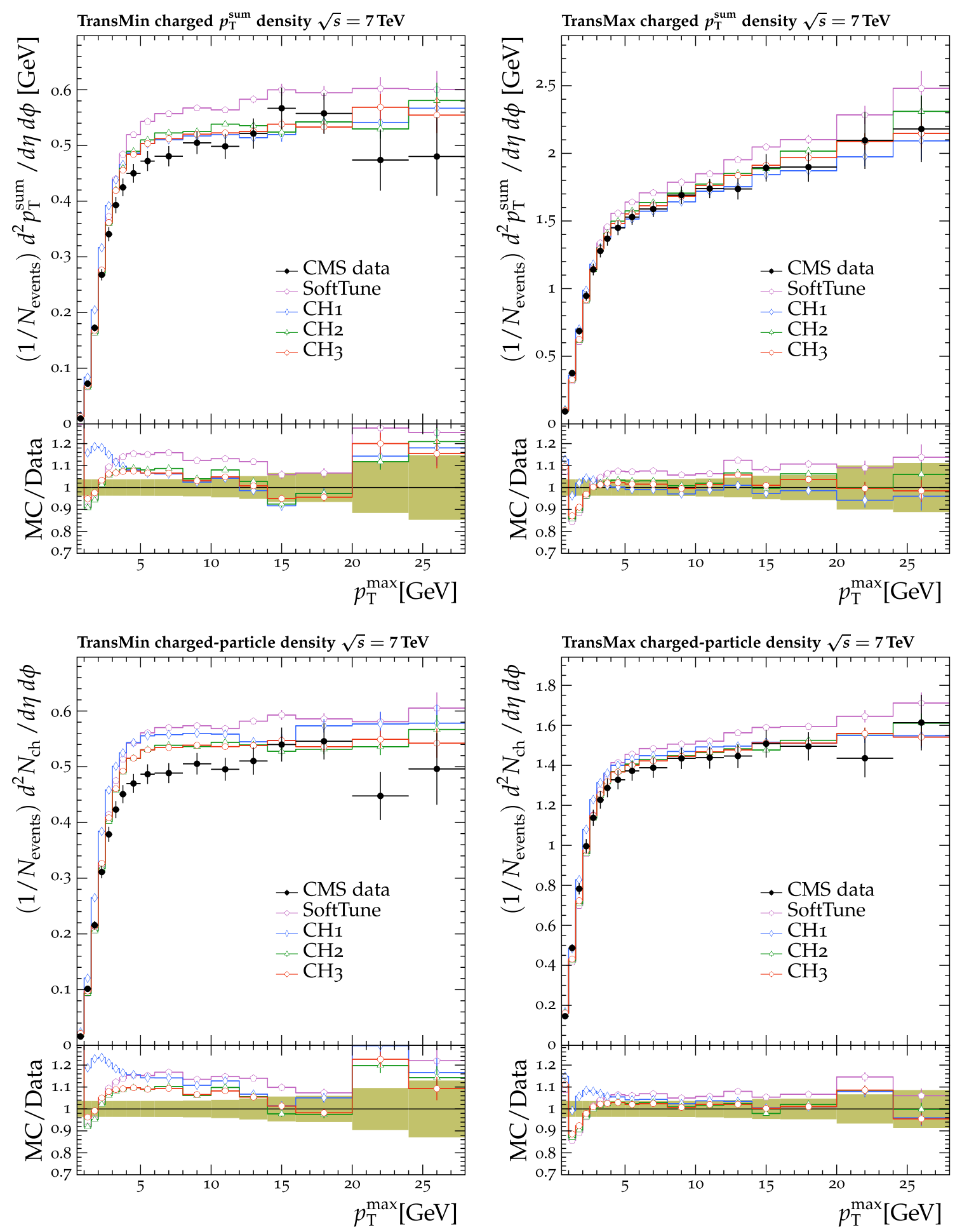

Fig. 4 The $p_{\mathrm{T}}^{\text {sum }}$ (upper) and $N_{\text {ch }}$ (lower) density distributions in the transMin (left) and transMax (right) regions, as a function of the $p_{\mathrm{T}}$ of the leading track, $p_{\mathrm{T}}^{\max }$ [23]. CMS MB data are compared with the predictions from HERWIG 7, with the SoftTune and $\mathrm{CH}$ tunes. The coloured

band in the ratio plot represents the total experimental uncertainty in the data. The vertical bars on the points for the different predictions represent the statistical uncertainties

tiplicity as a function of $\eta, \mathrm{d} N_{\mathrm{ch}} / \mathrm{d} \eta$, as measured by CMS at $\sqrt{s}=13 \mathrm{TeV}$ with zero magnetic field strength $(\mathrm{B}=0 \mathrm{~T})$ [27] is also used in the fitting procedure. The charged-particle

$p_{\mathrm{T}}$ and $\eta$ as measured by CMS in Ref. [28] are not considered here, since they are biased by predictions obtained with PYTHIA 6 [29], as discussed in Ref. [12]. 


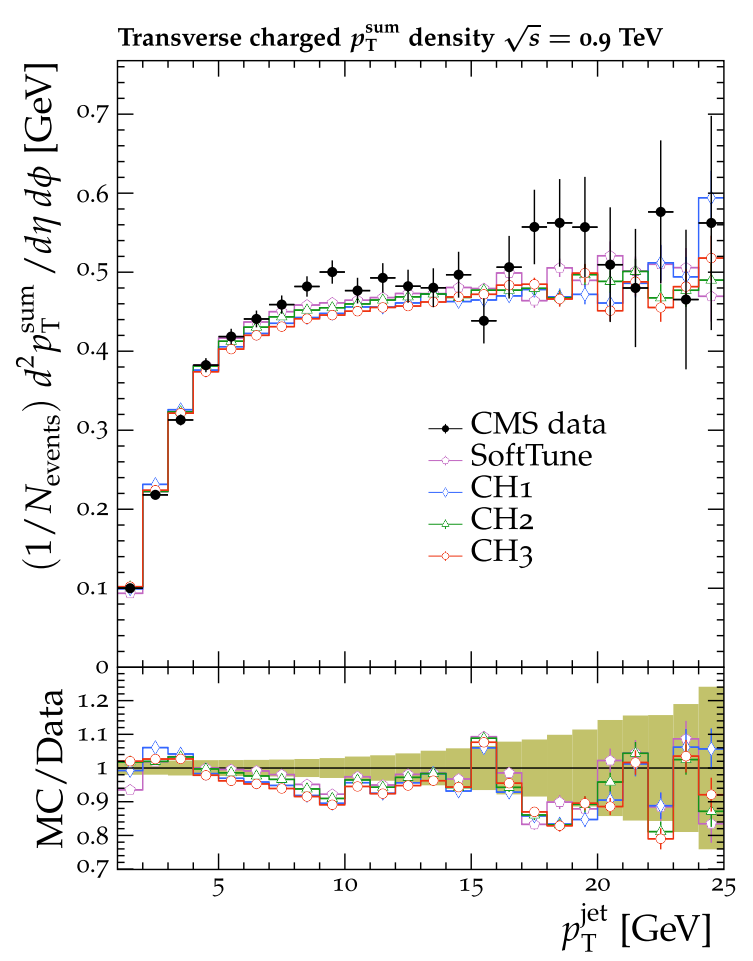

Fig. 5 The $p_{\mathrm{T}}^{\text {sum }}$ (left) and $N_{\mathrm{ch}}$ (right) density distributions in the transverse regions, as a function of the $p_{\mathrm{T}}$ of the leading track jet, $p_{\mathrm{T}}^{\mathrm{jet}}$ [25]. CMS MB data are compared with the predictions from HERWIG 7, with the SoftTune and $\mathrm{CH}$ tunes. The coloured band in the ratio plot repre-

The tuning is performed within the PROFESSOR (v1.4.0) framework [30]. Around 60 random choices of the parameters are made, and predictions for each of these choices are obtained using RIVET. Approximately 10 million MB events are generated for each choice of parameters, such that the uncertainty in the prediction in any bin is typically not larger than the uncertainty in the data in the same bin.

The fit is performed by minimising the $\chi^{2}$ function:

$\chi^{2}(p)=\sum_{\mathcal{O}} w_{\mathcal{O}} \sum_{i \in \mathcal{O}} \frac{\left(f^{i}(p)-\mathcal{R}_{i}\right)^{2}}{\Delta_{i}^{2}}$,

where $\mathcal{R}_{i}$ is the measured content of bin $i$ of the distribution of observable $\mathcal{O}$, while $f^{i}(p)$ is the predicted content in bin $i$, which is obtained by PROFESSOR from a parameterization of the dependence of the prediction on the tuning parameters $p$. The total uncertainty in the data and the simulated prediction in bin $i$ of a given observable is denoted by $\Delta_{i}^{2}$, and $w_{\mathcal{O}}$ is a weight that increases or decreases the importance of an observable $\mathcal{O}$ in the fit. The weight is typically set to $w_{\mathcal{O}}=1$. However, for the $\mathrm{CH} 1$ tune, where the PDF set used in the simulation of MPI and beam remnants is an NNLO set instead of an LO set, the weight is set to $w_{\mathcal{O}}=3$ for the $\mathrm{d} N_{\mathrm{ch}} / \mathrm{d} \eta$ distribution. This is the smallest weight that ensures the distribution is well described after the tuning. Beyond this, the parameters for the three tunes

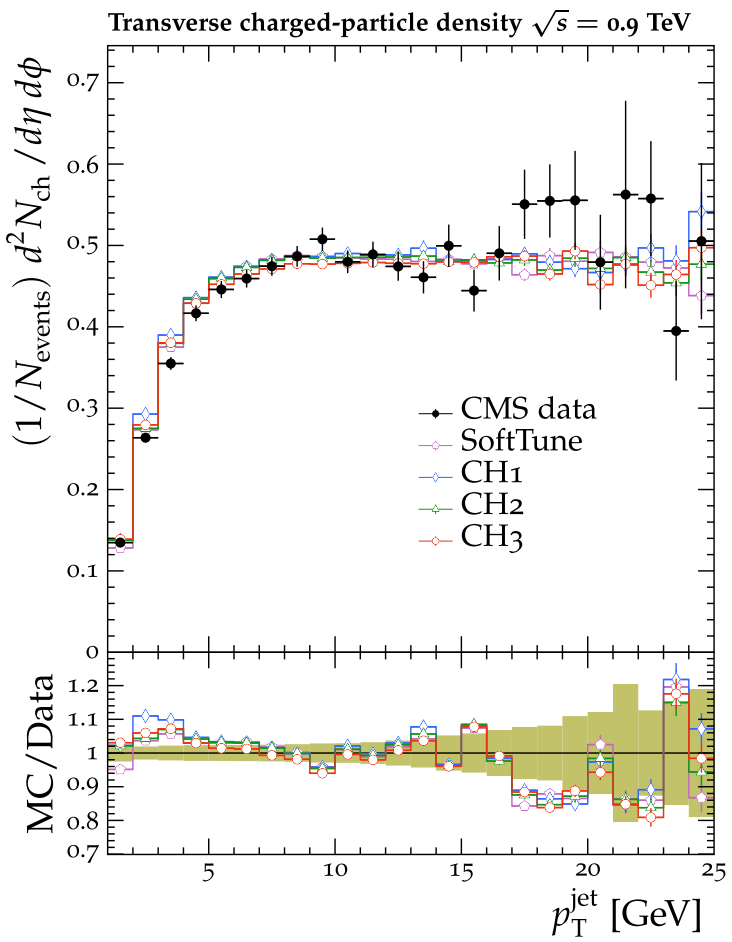

sents the total experimental uncertainty in the data. The vertical bars on the points for the different predictions represent the statistical uncertainties

and their predictions are stable with respect to a change in the weight assigned to the $\mathrm{d} N_{\mathrm{ch}} / \mathrm{d} \eta$ distribution in the fit. Correlations between the bins $i$ are not taken into account when minimising Eq. (5), because these were not available for the used input distributions. A third-order polynomial is used to parameterize the dependence of the prediction on the tuning parameters. Using a fourth-order polynomial to perform this interpolation between the 60 choices of parameters has a negligible effect on the outcome of the fits.

The number of degrees of freedom $\left(N_{\text {dof }}\right)$ in the fit is calculated as:

$N_{\text {dof }}=\frac{\left(\sum_{\mathcal{O}} \sum_{i \in \mathcal{O}} w_{\mathcal{O}}\right)^{2}}{\sum_{\mathcal{O}} \sum_{i \in \mathcal{O}} w_{\mathcal{O}}^{2}}-N_{\text {param }}$

where $N_{\text {param }}$ is the number of parameters being optimized in the fit.

\section{Results from the new HERWIG 7 tunes}

The tuned values of the parameters and the $\chi^{2}$ values from the fit, i.e. the minimum values of Eq. (5), divided by the $N_{\text {dof }}$ of the fit are shown in Table 2, along with the values of the parameters for the default tune SoftTune. The $N_{\text {dof }}$ in the fit is 118 for $\mathrm{CH} 1$, and 152 for $\mathrm{CH} 2$ and $\mathrm{CH} 3$. To provide 

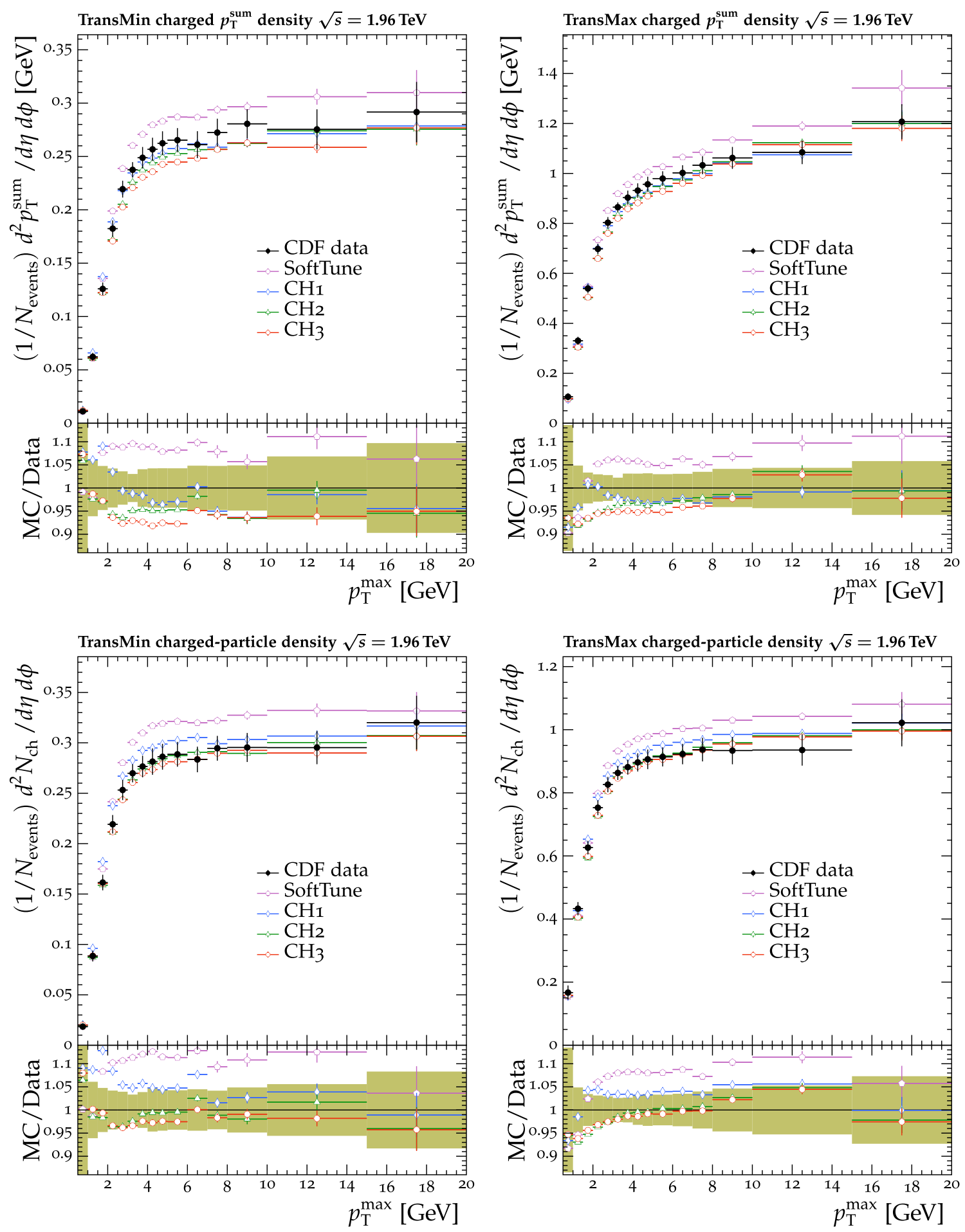

Fig. 6 The $p_{\mathrm{T}}^{\text {sum }}$ (upper) and $N_{\text {ch }}$ (lower) density distributions in the transMin (left) and transMax (right) regions, as a function of the $p_{\mathrm{T}}$ of the leading track, $p_{\mathrm{T}}^{\max }$ [31]. CDF MB data are compared with the predictions from HERWIG 7, with the SoftTune and $\mathrm{CH}$ tunes. The coloured

band in the ratio plot represents the total experimental uncertainty in the data. The vertical bars on the points for the different predictions represent the statistical uncertainties

a comparison between the compatibilities of the $\mathrm{CH}$ tunes and SoftTune with the data, the $\chi^{2} / N_{\text {dof }}$ corresponding to the prediction of SoftTune and the data is also shown with $N_{\text {dof }}$ set to 152 .

The values of the parameters of the MPI model are intertwined with each other since they are tuned simultaneously to reproduce the amount of UE activity observed in the data. Nonetheless, a general interpretation of the variations in the 

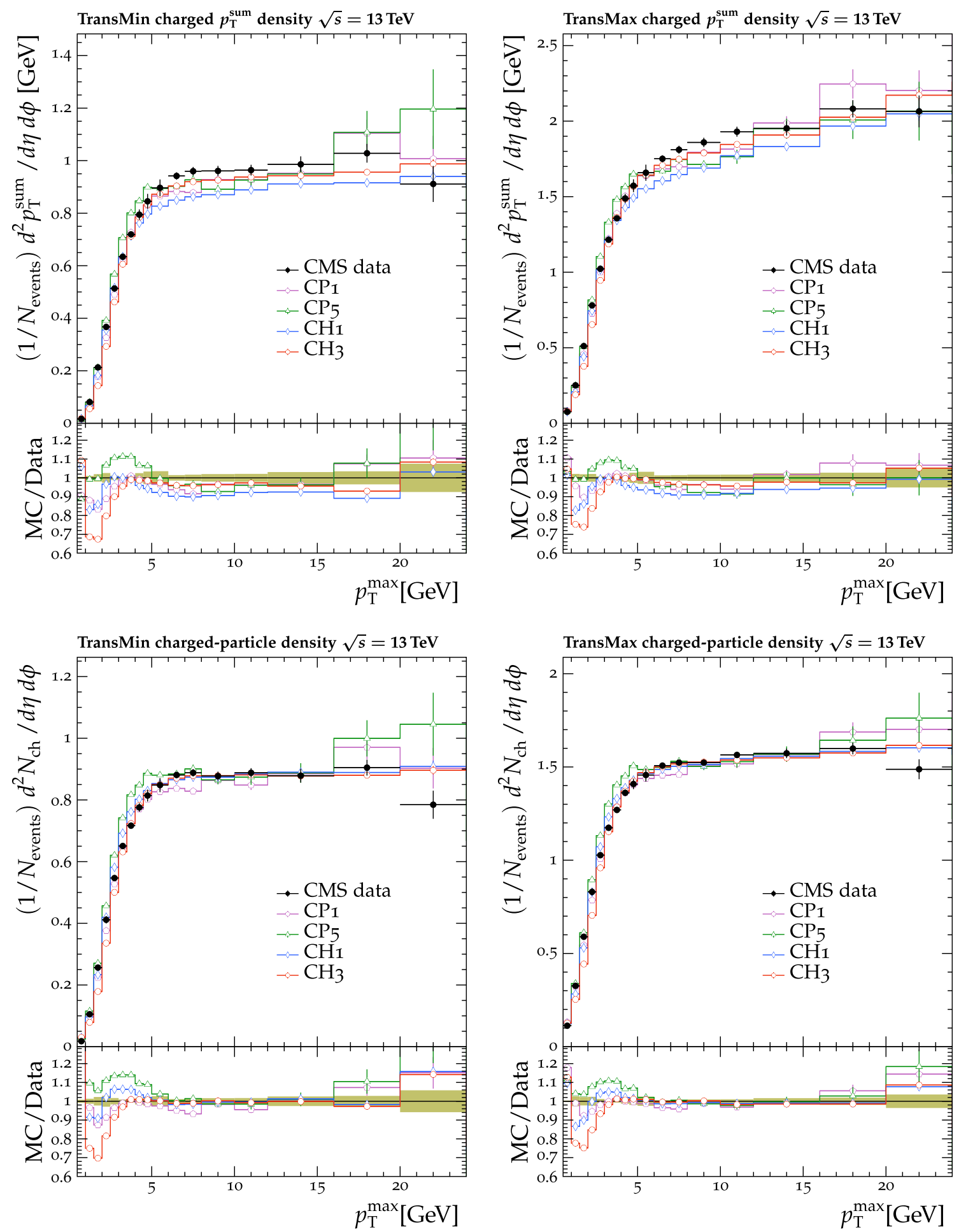

Fig. 7 The $p_{\mathrm{T}}^{\text {sum }}$ (upper) and $N_{\text {ch }}$ (lower) density distributions in the transMin (left) and transMax (right) regions, as a function of the $p_{\mathrm{T}}$ of the leading track, $p_{\mathrm{T}}^{\max }$ [24]. CMS MB data are compared with the predictions from HERWIG 7, with the $\mathrm{CH} 1$ and $\mathrm{CH} 3$ tunes, and from

PYTHIA 8 , with the $\mathrm{CP} 1$ and $\mathrm{CP} 5$ tunes. The coloured band in the ratio plot represents the total experimental uncertainty in the data. The vertical bars on the points for the different predictions represent the statistical uncertainties

tuned parameters for each tune can be distinguished. For example, the value of $p_{\perp, 0}^{\min }$ is lower for all three $\mathrm{CH}$ tunes than for SoftTune, and significantly lower for $\mathrm{CH} 1$, which increases the amount of MPI in an event compared to that with the tune SoftTune. 
The lower value of $b$ for all $\mathrm{CH}$ tunes further increases the contribution of MPI in collisions at $\sqrt{s}=13 \mathrm{TeV}$. Because of the lower values of $p_{\text {reco }}$, the amount of $\mathrm{CR}$ in the $\mathrm{CH}$ tunes is lower than in SoftTune. This also has the effect of increasing the overall amount of activity in the UE for the $\mathrm{CH}$ tunes. The value of $\mu^{2}$ for $\mathrm{CH} 2$ and $\mathrm{CH} 3$ is lower than the corresponding value for SoftTune. Even though a lower value of $\mu^{2}$ would lead to a lower amount of MPI in a given event, the combined effect of the parameters of the $\mathrm{CH}$ tunes results in a larger amount of MPI compared with SoftTune.

The tuned parameters of $\mathrm{CH} 2$ and $\mathrm{CH} 3$ are fairly similar, as are the values of $\chi^{2} / N_{\text {dof }}$ of these two tunes, indicating that the choice of $\alpha_{\mathrm{S}}\left(m_{\mathrm{Z}}\right)$ used when deriving the LO PDF set in the simulation of MPI does not have a large effect. The parameters for the tune $\mathrm{CH} 1$ differ from those for the tunes $\mathrm{CH} 2$ and $\mathrm{CH} 3$, and the value of $\chi^{2} / N_{\text {dof }}$ is larger, implying that using an LO PDF set is somewhat preferred over an NNLO PDF set for the simulation of MPI. In the following, the predictions from the three $\mathrm{CH}$ tunes are compared with the data used in the tuning procedure. These predictions are obtained by generating events with the corresponding parameters shown in Table 2 rather than from the parameterization of the tune parameters used in the fit.

Figure 2 shows the normalized $\mathrm{d} N_{\mathrm{ch}} / \mathrm{d} \eta$ of charged hadrons as a function of $\eta$ at $13 \mathrm{TeV}$ in MB events. Only the predictions for SoftTune deviate significantly from the data, and underestimate the $\mathrm{d} N_{\mathrm{ch}} / \mathrm{d} \eta$ in data by $10-18 \%$. The $\mathrm{CH}$ tunes each provide a slightly different prediction, but all have a similar level of agreement with the data. The $\mathrm{CH}$ tunes compared with SoftTune predict an increase in the UE activity, which is observed.

Figure 3 shows the normalized $p_{\mathrm{T}}^{\text {sum }}$ and $N_{\mathrm{ch}}$ densities as a function of $p_{\mathrm{T}}^{\max }$ with comparisons from SoftTune and the $\mathrm{CH}$ tunes for both transMin and transMax. The predictions of SoftTune and the $\mathrm{CH} 2, \mathrm{CH} 3$ tunes are broadly similar, and give a good description the data in the plateau region $\left(p_{\mathrm{T}}^{\max } \gtrsim\right.$ $4 \mathrm{GeV}$ ). In the rising part of the spectrum, the predictions from the tunes $\mathrm{CH} 2, \mathrm{CH} 3$, and SoftTune deviate from the data in some bins by up to $40 \%$. The $\mathrm{CH} 3$ tune provides the best predictions in the rising region of the spectrum. However, only the region $p_{\mathrm{T}}^{\max }>3 \mathrm{GeV}$ was included in the tuning procedure, because the region $p_{\mathrm{T}}^{\max }<3 \mathrm{GeV}$ is dominated by diffractive processes whose model parameters are not used in the fit.

The effect of using an NNLO PDF, instead of an LO PDF, in the simulation of MPI is seen from the predictions with the tune $\mathrm{CH} 1$ in Fig. 3. This tune provides a good description of the $N_{\mathrm{ch}}$ distributions in both the transMin and transMax regions, and is typically within $10 \%$ of the data. However, the tune $\mathrm{CH} 1$ does not simultaneously provide a good description of the $p_{\mathrm{T}}^{\text {sum }}$ distributions in either the transMin or transMax region, with a $10 \%$ difference to the data in the plateau region of the corresponding transMax distribution.

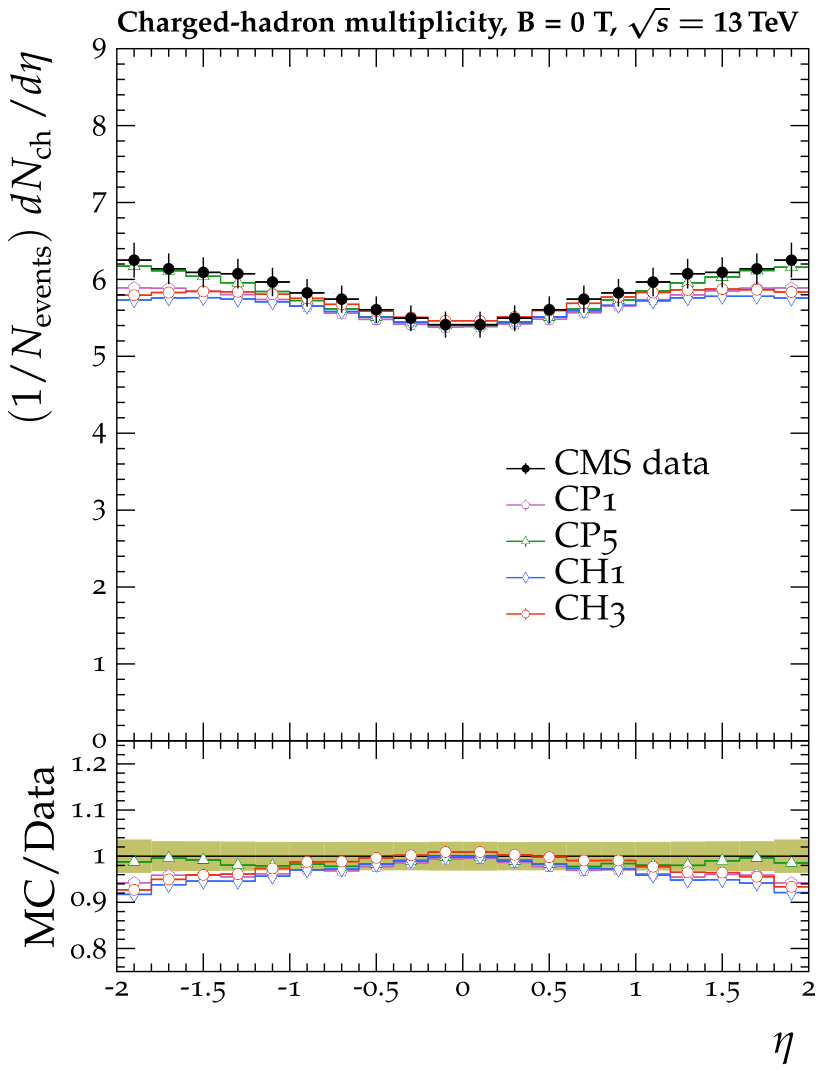

Fig. 8 The normalized $\mathrm{d} N_{\mathrm{ch}} / \mathrm{d} \eta$ of charged hadrons as a function of $\eta$ [27]. CMS MB data are compared with the predictions from HERWIG 7, with the $\mathrm{CH} 1$ and $\mathrm{CH} 3$ tunes, and from PYTHIA 8 , with the $\mathrm{CP} 1$ and $\mathrm{CP} 5$ tunes. The coloured band in the ratio plot represents the total experimental uncertainty in the data. The vertical bars on the points for the different predictions represent the statistical uncertainties

Table 3 Parameters of the central, "up", and "down" variations of the $\mathrm{CH} 3$ tune

\begin{tabular}{llll}
\hline & $\mathrm{CH} 3$ & & \\
\cline { 2 - 4 } & Down & Central & Up \\
\hline$p_{\perp, 0}^{\min }(\mathrm{GeV})$ & 2.349 & 3.040 & 3.382 \\
$b$ & 0.298 & 0.136 & 0.328 \\
$\mu^{2}\left(\mathrm{GeV}^{-2}\right)$ & 1.160 & 1.284 & 1.539 \\
$p_{\text {reco }}$ & 0.641 & 0.471 & 0.191 \\
\hline
\end{tabular}

Figure 4 shows the normalized $N_{\mathrm{ch}}$ and $p_{\mathrm{T}}^{\text {sum }}$ densities as a function of $p_{\mathrm{T}}^{\max }$ using UE data at $7 \mathrm{TeV}$ and compared with various tunes. In the transMax region, the predictions from the $\mathrm{CH}$ tunes describe the data well, with at most a $15 \%$ discrepancy at low $p_{\mathrm{T}}^{\max }$. In the transMin region, the predictions from all tunes deviate from the data at intermediate values of $p_{\mathrm{T}}^{\max } \approx 3-8 \mathrm{GeV}$. The deviation is up to $\approx 10 \%$ for the $\mathrm{CH} 2$ and $\mathrm{CH} 3$ tunes, whereas the difference between data and the tunes SoftTune and $\mathrm{CH} 1$ is larger than this. The prediction of $\mathrm{CH} 1$ deviates further from the data at lower values of $p_{\mathrm{T}}^{\max }$.

The predictions are compared with UE data at $\sqrt{s}=$ $0.9 \mathrm{TeV}$ to normalized $p_{\mathrm{T}}^{\text {sum }}$ densities in the transverse 

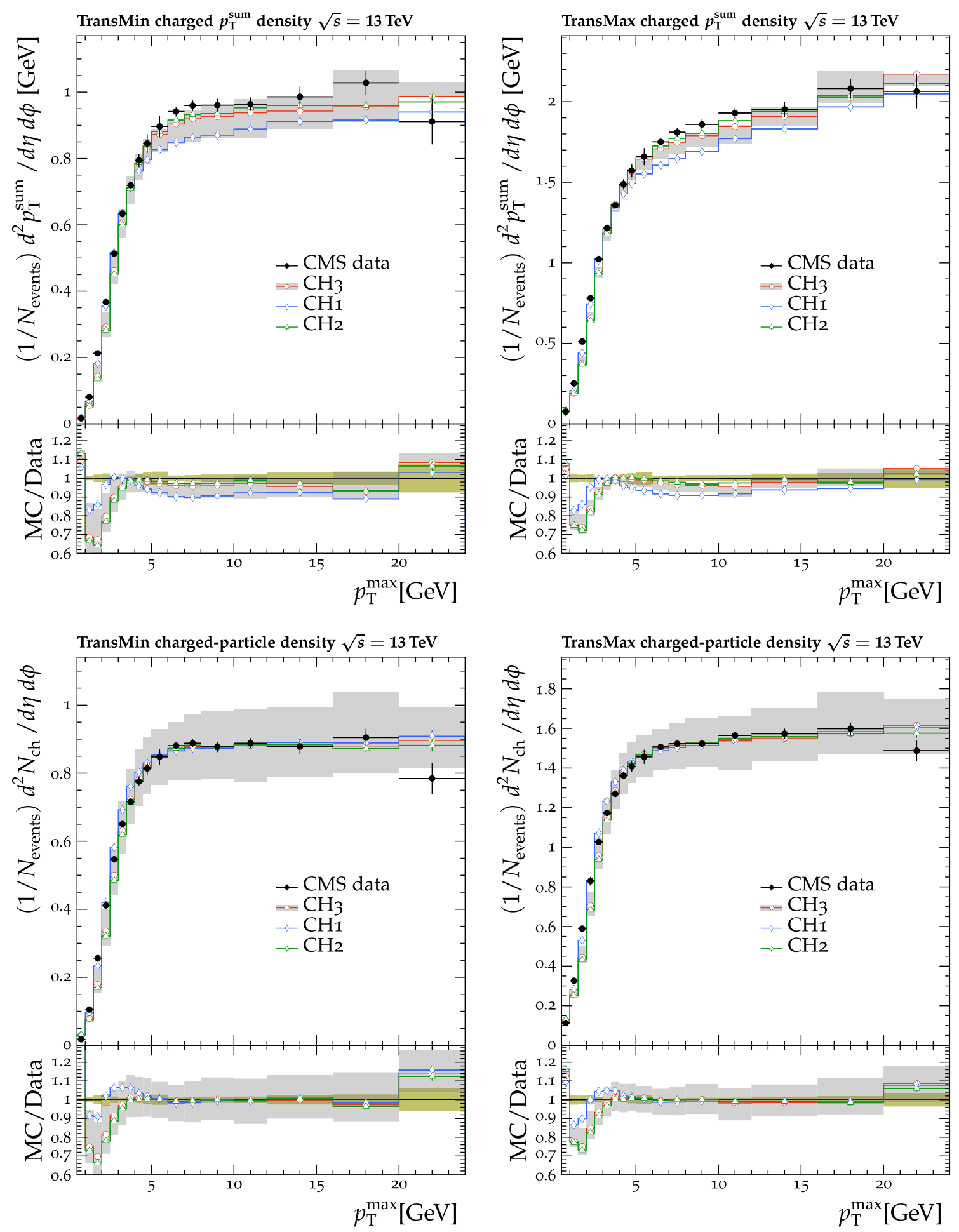

Fig. 9 The $p_{\mathrm{T}}^{\text {sum }}$ (upper) and $N_{\text {ch }}$ (lower) density distributions in the transMin (left) and transMax (right) regions, as a function of the $p_{\mathrm{T}}$ of the leading track, $p_{\mathrm{T}}^{\max }$ [24]. CMS MB data are compared with the predictions from HERWIG 7 , with the $\mathrm{CH}$ tunes. The coloured band in

regions in Fig. 5. All tunes provide a similar prediction of the observables above $p_{\mathrm{T}}^{\text {jet }}>4 \mathrm{GeV}$, and agree with the data. the ratio plot represents the total experimental uncertainty in the data. The vertical bars on the points for the different predictions represent the statistical uncertainties. The grey-shaded band corresponds to the envelope of the "up" and "down" variations of the $\mathrm{CH} 3$ tune

$p_{\mathrm{T}}^{\text {jet }}$, with the tunes $\mathrm{CH} 2$ and $\mathrm{CH} 3$ providing a better description of the data compared to the tunes $\mathrm{CH} 1$ and SoftTune. 
Figure 6 shows comparisons of the normalized $p_{\mathrm{T}}^{\text {sum }}$ and $N_{\text {ch }}$ densities using tune predictions with UE data collected by the CDF experiment at the Fermilab Tevatron at $\sqrt{s}=1.96 \mathrm{TeV}$ [31]. The $\mathrm{CH}$ tunes describe the distributions in both transMin and transMax well, however the $\mathrm{CH} 3$ tune underestimates the $p_{\mathrm{T}}^{\text {sum }}$ data somewhat at $p_{\mathrm{T}}^{\max }<10 \mathrm{GeV}$, in both the transMin and transMax regions. Although these data were not used in deriving any of the tunes considered here, they validate that the energy dependence of the new tunes is correctly modelled. The tune SoftTune overestimates the data by $\approx 5-15 \%$ in all distributions. Additional comparisons of the predictions of HERWIG 7 with the various tunes using MB data from the ATLAS experiment, which were used in deriving SoftTune, are shown in Appendix A. One notable difference between the distribution of $\mathrm{d} N_{\mathrm{ch}} / \mathrm{d} \eta$ shown in Fig. 2 and the one shown in Fig. 24 is that the former includes all charged particles, whereas the latter includes only charged particles with $p_{\mathrm{T}}>500 \mathrm{MeV}$.

Based on the comparisons shown in this section, the tunes $\mathrm{CH} 2$ and $\mathrm{CH} 3$ both provide a similar description of the data, indicating that the choice between the two LO PDFs used for the simulation of MPI and remnant handling has little effect on the predictions. These two PDFs are both LO PDFs, but a value of $\alpha_{\mathrm{S}}\left(m_{\mathrm{Z}}\right)=0.118$ is used in deriving the PDF used with $\mathrm{CH} 2$, and a value of $\alpha_{\mathrm{S}}\left(m_{\mathrm{Z}}\right)=0.130$ is assumed for the PDF used with CH3. As stated in Sect. 3, $\alpha_{\mathrm{S}}\left(m_{\mathrm{Z}}\right)=0.118$ is used in all parts of the HERWIG 7 simulation for the three $\mathrm{CH}$ tunes. From Table 2, the $\chi^{2} / N_{\mathrm{dof}}$ for the tune $\mathrm{CH} 2$ is slightly lower than that for the tune $\mathrm{CH} 3$. However, the use of the LO PDF in the tune CH3, which was derived with $\alpha_{\mathrm{S}}\left(m_{\mathrm{Z}}\right)=0.130$, is consistent with the value of $\alpha_{\mathrm{S}}\left(m_{\mathrm{Z}}\right)$ typically associated with LO PDFs and therefore is a preferred choice over the tune $\mathrm{CH} 2$. Both of the tunes $\mathrm{CH} 2$ and $\mathrm{CH} 3$ provide a better description of the data than the tune CH1, where the NNLO NNPDF3.1 PDF was used for the simulation of MPI and remnant handling. This suggests that the use of the LO NNPDF3.1 PDF is preferred in this aspect of the HERWIG 7 simulation, even though the gluon PDF in both the LO and NNLO PDF sets are positive at low energy scales, as discussed earlier.

In Fig. 7 the normalized $N_{\text {ch }}$ and $p_{\mathrm{T}}^{\text {sum }}$ density predictions of the UE data at $\sqrt{s}=13 \mathrm{TeV}$ show a comparison of the $\mathrm{CH} 1$ and $\mathrm{CH} 3$ tunes with those obtained from the PYTHIA 8 (version 8.230) using the tunes CP1 and CP5 [19]. The tune $\mathrm{CH} 2$ is not displayed, because its prediction is similar to the one of the tune $\mathrm{CH} 3$. The $\mathrm{CP} 1$ tune uses an LO NNPDF3.1 PDF set in all aspects of the PYTHIA 8 simulation, an $\alpha_{\mathrm{S}}\left(m_{\mathrm{Z}}\right)$ value of 0.130 in the simulation of MPI and hard scattering, and an $\alpha_{\mathrm{S}}\left(m_{\mathrm{Z}}\right)$ value of 0.1365 for the simulation of initial- and final-state radiation. The CP5 tune uses an NNLO PDF set with an $\alpha_{\mathrm{S}}\left(m_{\mathrm{Z}}\right)$ value of 0.118 in all aspects of simulation. The choice of the PDF

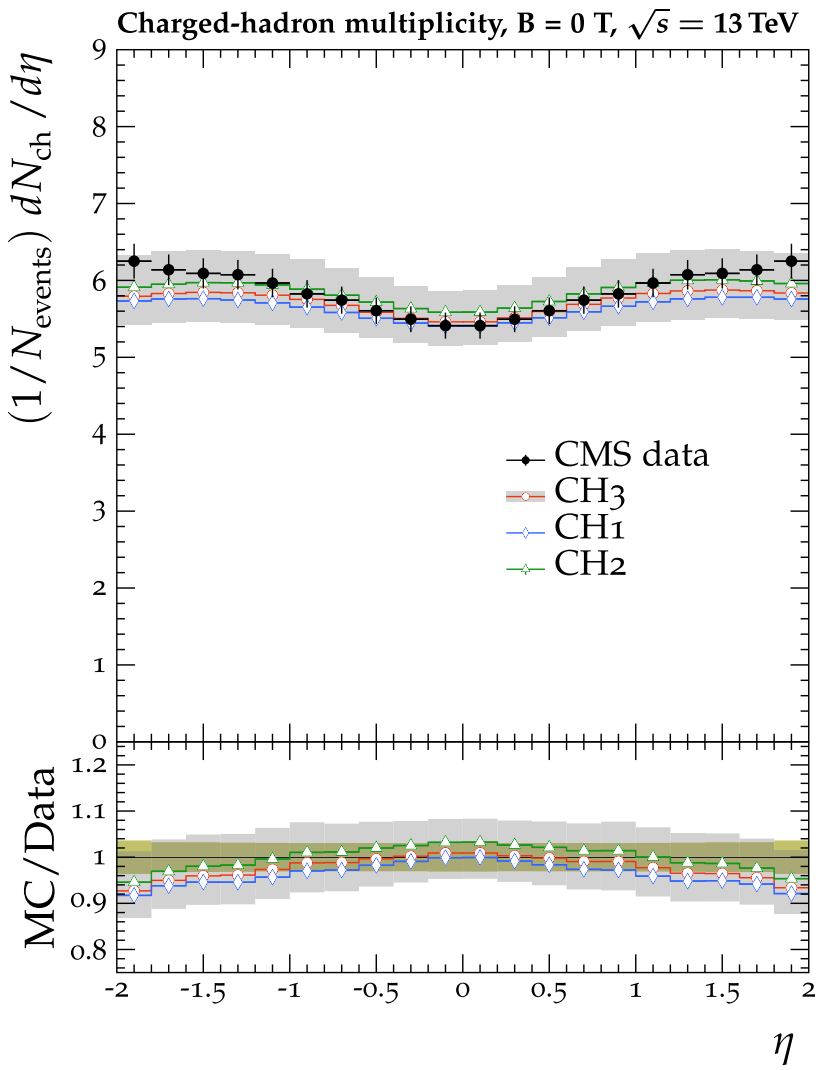

Fig. 10 The normalized $\mathrm{d} N_{\mathrm{ch}} / \mathrm{d} \eta$ of charged hadrons as a function of $\eta$ [27]. CMS MB data are compared with the predictions from HERWIG 7, with the $\mathrm{CH}$ tunes. The coloured band in the ratio plot represents the total experimental uncertainty in the data. The vertical bars on the points for the different predictions represent the statistical uncertainties. The grey-shaded band corresponds to the envelope of the "up" and "down" variations of the $\mathrm{CH} 3$ tune

set and $\alpha_{\mathrm{S}}\left(m_{\mathrm{Z}}\right)$ value in the CP5 tune is the same as the CH1 HERWIG 7 tune. Although all the predictions show a reasonable agreement with the data in the plateau region of the UE distributions, the use of an LO PDF for MPI and remnant handling in $\mathrm{CH} 3$ provides a slightly improved description of the $p_{\mathrm{T}}^{\text {sum }}$ data compared to using an NNLO PDF in $\mathrm{CH} 1$. This differs from the predictions of PYTHIA 8, where the use of an LO and NNLO PDF for simulating MPI give a similar description of the data in this region. Each prediction exhibits different behaviour at low $p_{\mathrm{T}}^{\max }$. None of the HERWIG 7 Or PYTHIA 8 tunes provides a perfect description of the data at low $p_{\mathrm{T}}^{\max }$, since they exhibit at least a $10 \%$ difference between any one of the tunes and the data. Figure 8 shows a similar comparison for the $\eta$ distribution of charged hadrons at $13 \mathrm{TeV}$. The prediction from $\mathrm{CP} 5$ provides a better description of the data compared with the other tunes at larger values of $|\eta|$. The predictions from the HERWIG 7 tunes show a closer behaviour to the $\mathrm{CP} 1$ tune in this distribution. 

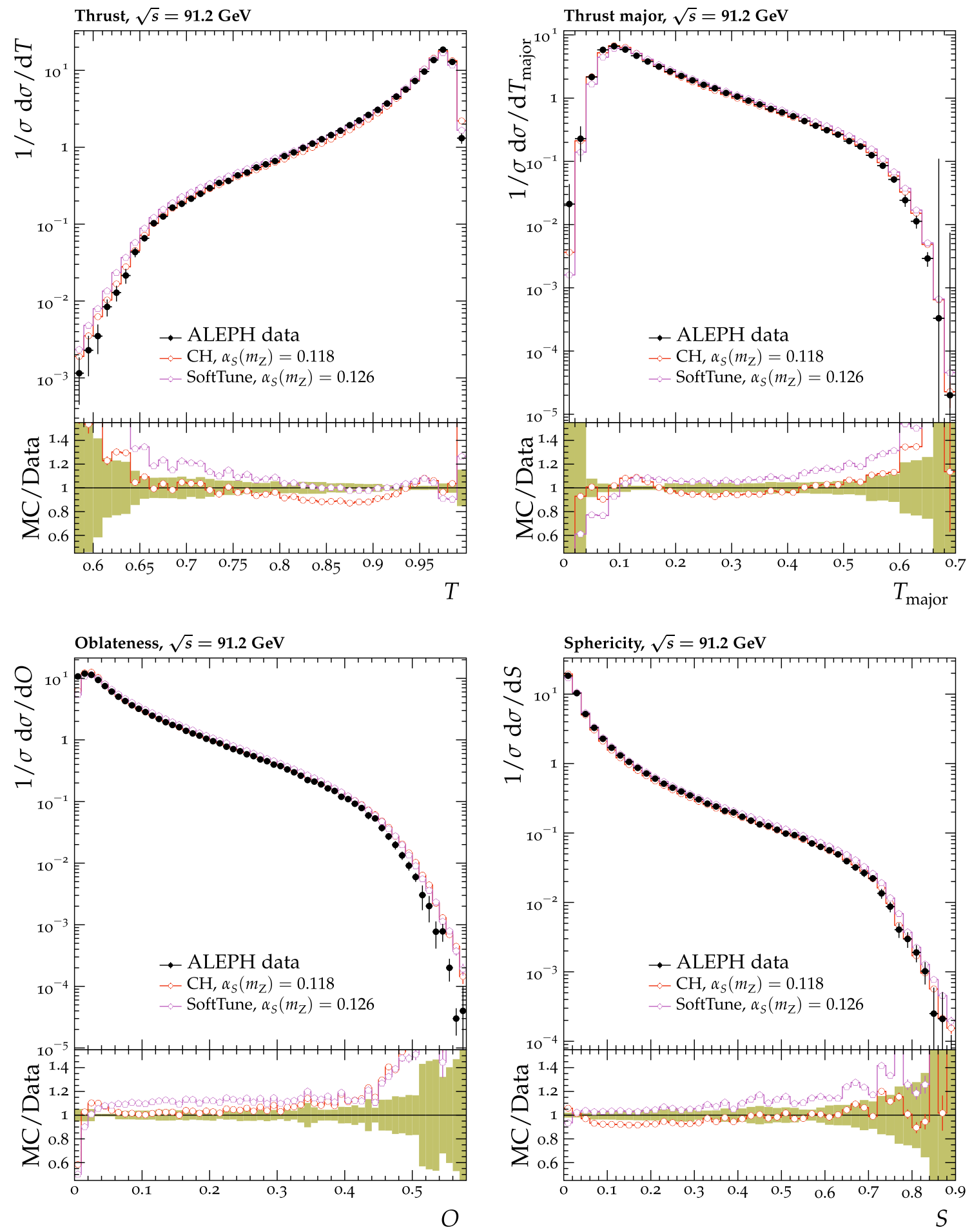

Fig. 11 Normalized differential cross sections for $\mathrm{e}^{-} \mathrm{e}^{+}[32]$ as a function of the variables $T$ (upper left), $T_{\text {major }}$ (upper right), $O$ (lower left), and $S$ (lower right) for ALEPH data at $\sqrt{s}=91.2 \mathrm{GeV}$. ALEPH data are compared with the predictions from HERWIG 7 using the SoftTune and

$\mathrm{CH}$ tunes. The coloured band in the ratios of the different predictions from simulation to the data represents the total experimental uncertainty in the data

\section{Uncertainties in the HERWIG 7 tunes}

Alternative tunes are derived in this section that provide an approximation to the uncertainties in the parameters of the

tune $\mathrm{CH} 3$. These are obtained from the eigentunes provided by PROFESSOR. These eigentunes are variations of the tuned parameters along the maximally independent directions in the parameter space by an amount corresponding to a change 

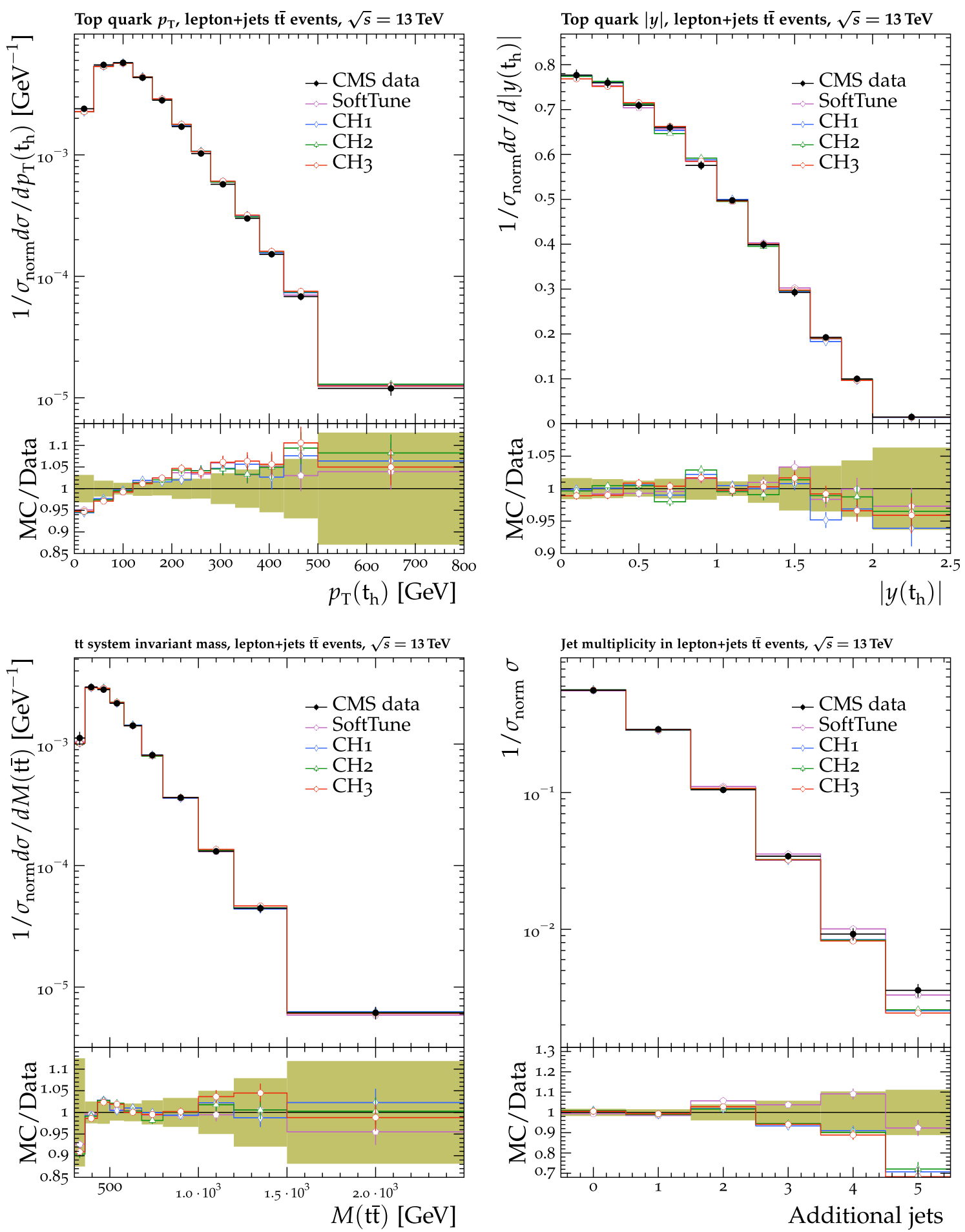

Fig. 12 The differential cross sections are shown as functions of: the $p_{\mathrm{T}}$ (upper left) and rapidity (upper right) of the hadronically decaying top quark; the invariant mass of the t' system (lower left); the additional jet multiplicity (lower right) [38]. CMS tit data are compared with the

predictions from POWHEG + HERWIG 7, with the SoftTune, CH1, CH2, and $\mathrm{CH} 3$ tunes. The coloured band in the ratio plot represents the total experimental uncertainty in the data. The vertical bars on the points for the different predictions represent the statistical uncertainties

in the $\chi^{2}\left(\Delta \chi^{2}\right)$ equal to the optimal $\chi^{2}$ of the fit. Because a change $\Delta \chi^{2}$ in Eq. (5) does not result in a variation with a meaningful statistical interpretation, the value of $\Delta \chi^{2}$ is

chosen in an empirical way. The change $\Delta \chi^{2}=\chi^{2}$, which is suggested by the PROFESSOR Collaboration, results in variations that are similar in magnitude to the uncertainties in 


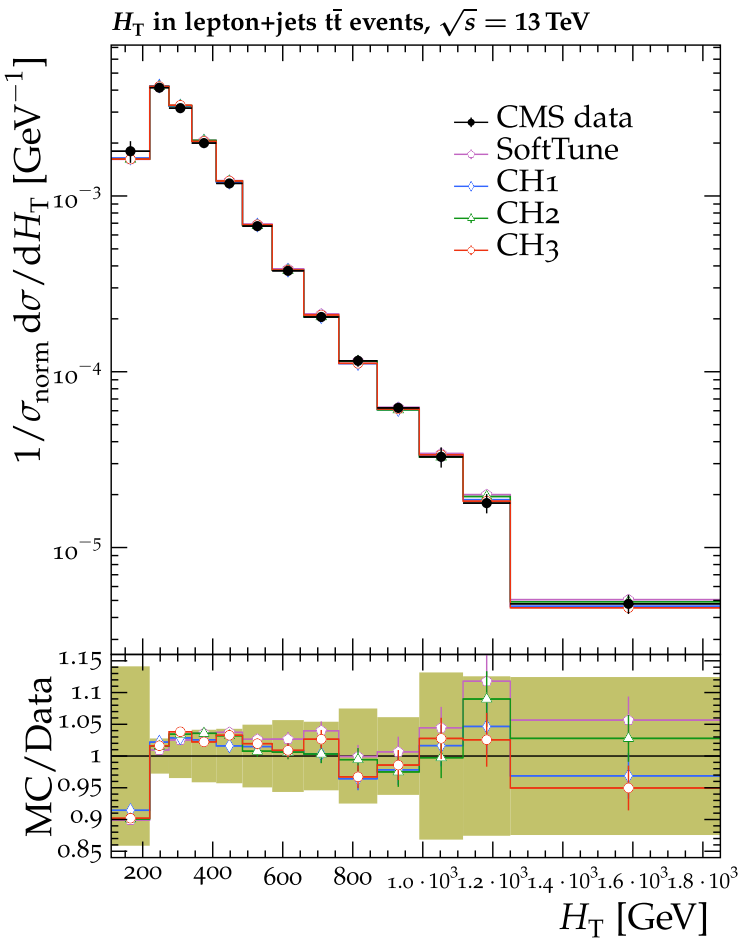

Fig. 13 The differential cross sections are shown as functions of $H_{\mathrm{T}}$ (left) and $p_{\mathrm{T}}^{\text {miss }}$ (right) [41]. CMS t't data are compared with the predictions from POWHEG + HERWIG 7, with the SoftTune, CH1, CH2, and

the fitted data points and judged to provide a reasonable set of variations that reflect the combined statistical and systematic uncertainty in the model parameters. A consequence of this adopted procedure is that the uncertainty may not necessarily cover the data in every bin. If the uncertainties in the fitted data points were uncorrelated between themselves, then the magnitude of the uncertainties in the data points depends on their bin widths. For the data used in the fit, the uncertainties are typically dominated by uncertainties that are correlated between the bins. However, the uncertainties in the data points at high $p_{\mathrm{T}}^{\max }$ and $p_{\mathrm{T}}^{\text {jet }}$, e.g. $p_{\mathrm{T}}^{\max } \gtrsim 10 \mathrm{GeV}$ for the UE observables at $\sqrt{s}=13 \mathrm{TeV}$, are dominated by statistical uncertainties, which are uncorrelated between bins. This introduces some dependence of the eigentunes on the bin widths of the data used in the fit.

The variations of the tunes provided by the eight eigentunes are reduced to two variations, as explained below, one "up" and one "down" variation. The "up" variation is obtained by considering the positive differences in each bin between each eigentune and the central prediction of the $\mathrm{CH} 3$ tune for the distributions used in the tuning procedure. The difference for each eigentune is summed in quadrature. Similarly, the "down" variation is obtained by considering the negative differences between the eigentunes and the central predictions. The two variations are then fitted, using the same procedure described in Sect. 3 to obtain a set of tune param-

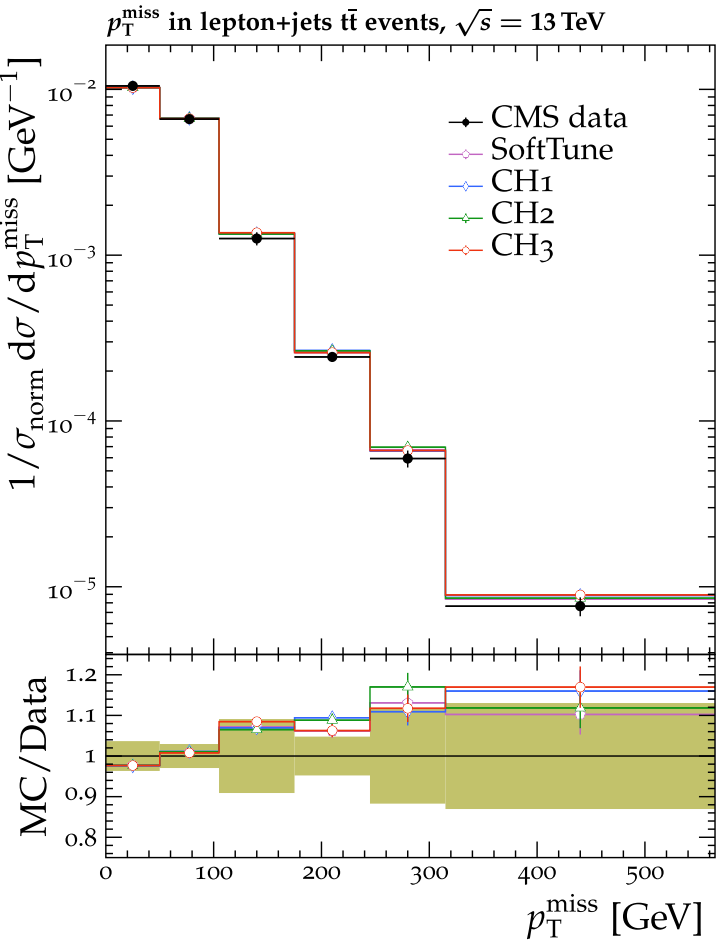

$\mathrm{CH} 3$ tunes. The coloured band in the ratio plot represents the total experimental uncertainty in the data. The vertical bars on the points for the different predictions represent the statistical uncertainties

eters that describe these two variations. The parameters of the two variations are shown in Table 3 . The values of each parameter of the variations do not necessarily encompass the corresponding values of the $\mathrm{CH} 3$ tune, as a result of the method of determining the variations from the differences between several eigentunes. The two variations accurately replicate the combination of all eigentunes, i.e. the sum in quadrature of all positive or negative differences with respect to the central prediction. By using these variations, the uncertainties in the tune $\mathrm{CH} 3$ are estimated by considering only two variations of the tune parameters, rather than eight variations. However, the correlations between bins of an observable for each of the eight individual variations are not known when considering only the "up" and "down" variations.

Figures 9 (normalized $p_{\mathrm{T}}^{\text {sum }}$ and $N_{\text {ch }}$ densities) and 10 (normalized $\mathrm{d} N_{\mathrm{ch}} / \mathrm{d} \eta$ ) show predictions from the $\mathrm{CH}$ tunes. The grey-shaded band corresponds to the envelope of the "up" and "down" variations, for the UE and MB observables used in the tuning procedure. The differences between the $\mathrm{CH} 1$ and $\mathrm{CH} 2$ predictions and those from $\mathrm{CH} 3$ are within the uncertainty of $\mathrm{CH} 3$, except for a small deviation at low $p_{\mathrm{T}}^{\max }$. 

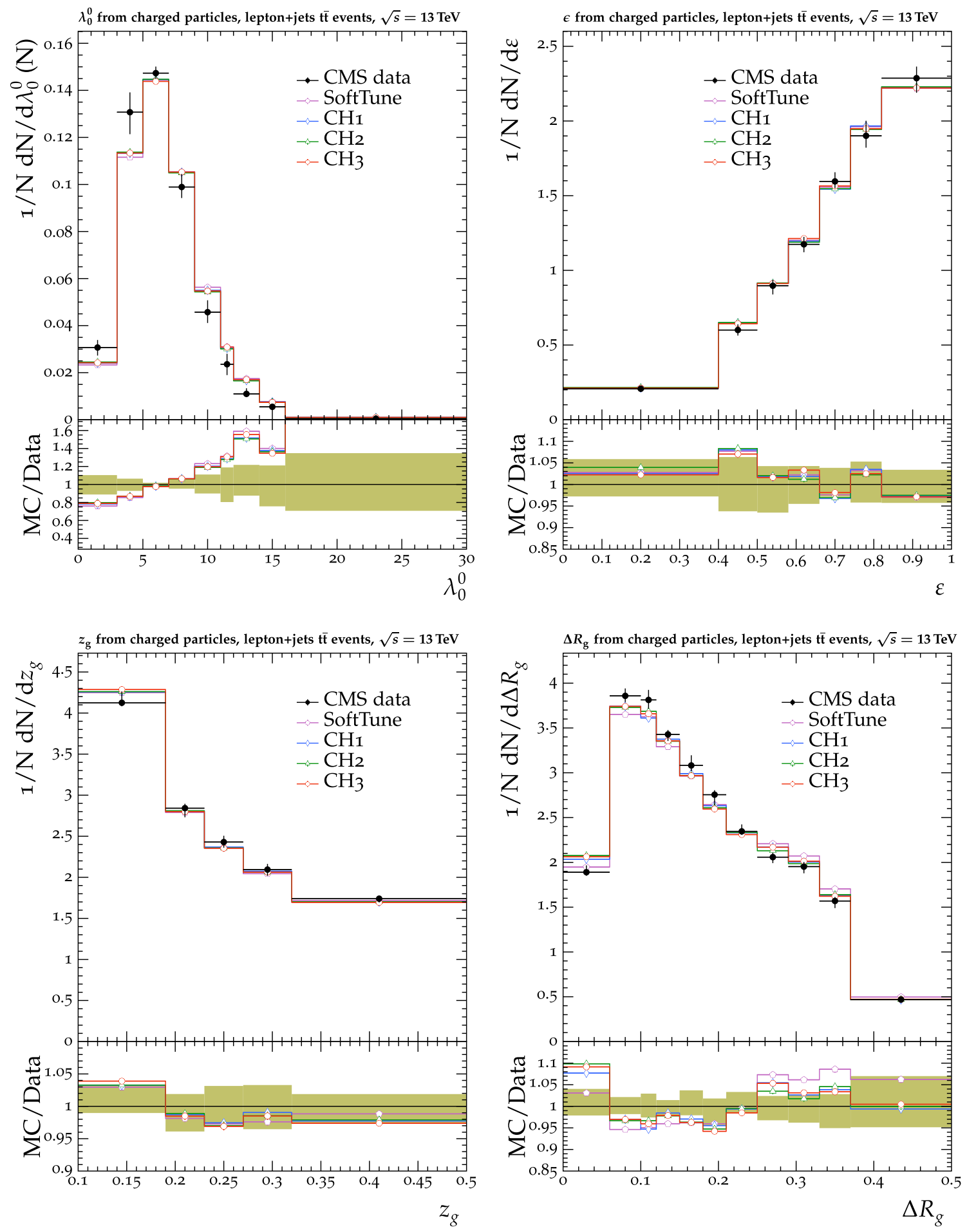

Fig. 14 The normalized jet substructure observables in single-lepton events: the charged-particle multiplicity (upper left); the eccentricity (upper right); the groomed momentum fraction (lower left); and the angle between the groomed subjects (lower right) [42]. CMS tt data are compared with the predictions from POWHEG + HERWIG 7, with the

SoftTune, $\mathrm{CH} 1, \mathrm{CH} 2$, and $\mathrm{CH} 3$ tunes. The coloured band in the ratio plot represents the total experimental uncertainty in the data. The vertical bars on the points for the different predictions represent the statistical uncertainties 

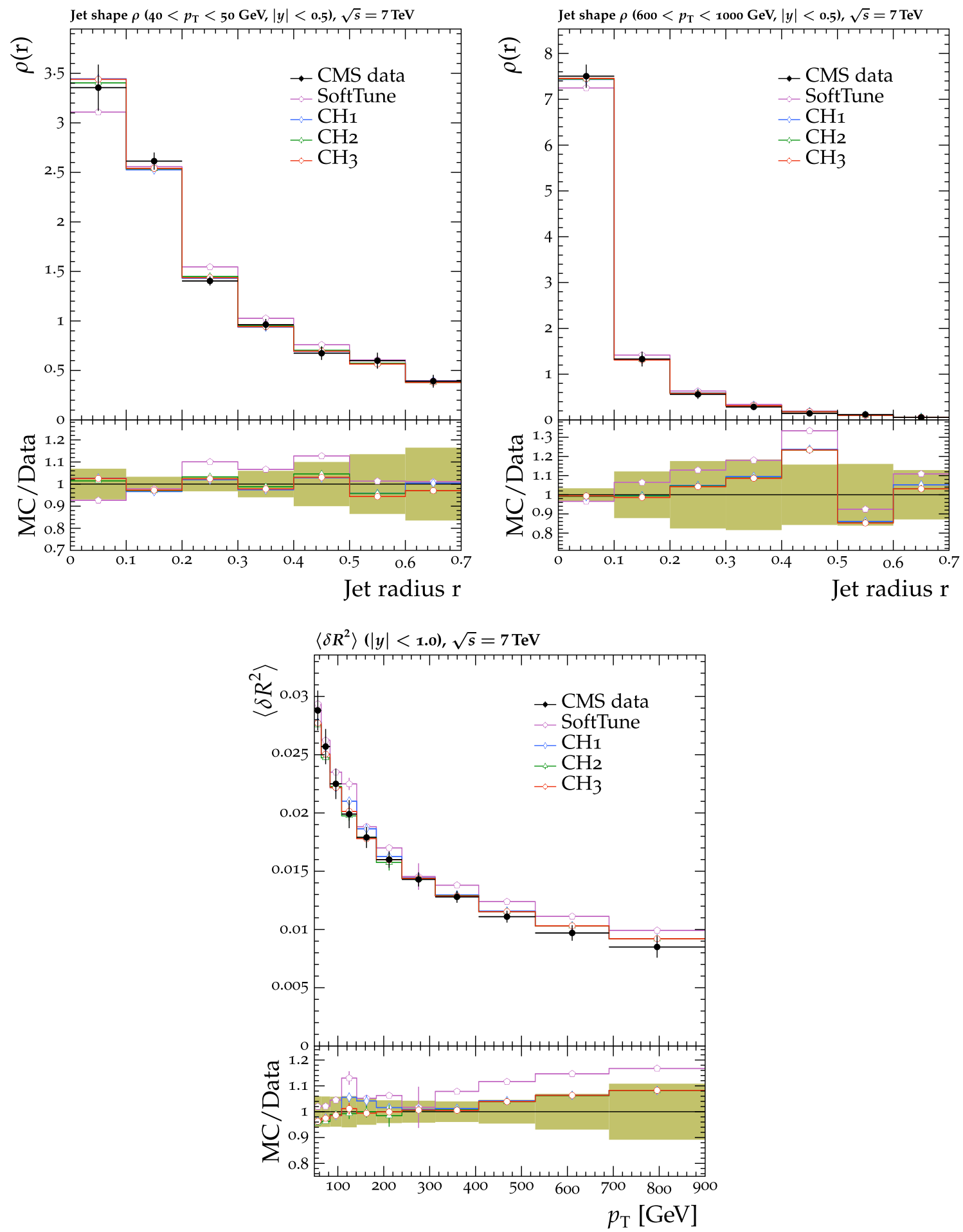

Fig. 15 The differential jet shape $\rho$ (r) (upper left and right) and the second moment of the jet transverse width $\left\langle\delta R^{2}\right\rangle$ in inclusive jet events [43]. CMS inclusive jet data are compared with the predictions from HERWIG 7, with the SoftTune and CH tunes. The coloured band in the

\section{Comparison with LEP data}

HERWIG 7 predictions are obtained in this section for event shape observables measured in LEP electron-positron col- ratio plot represents the total experimental uncertainty in the data. The vertical bars on the points for the different predictions represent the statistical uncertainties

lisions at $\sqrt{s}=91.2 \mathrm{GeV}$. The predictions are obtained using NLO MEs implemented within HERWIG 7. Figure 11 shows the thrust $(T)$, thrust major $\left(T_{\text {major }}\right)$, oblateness $(O)$, 


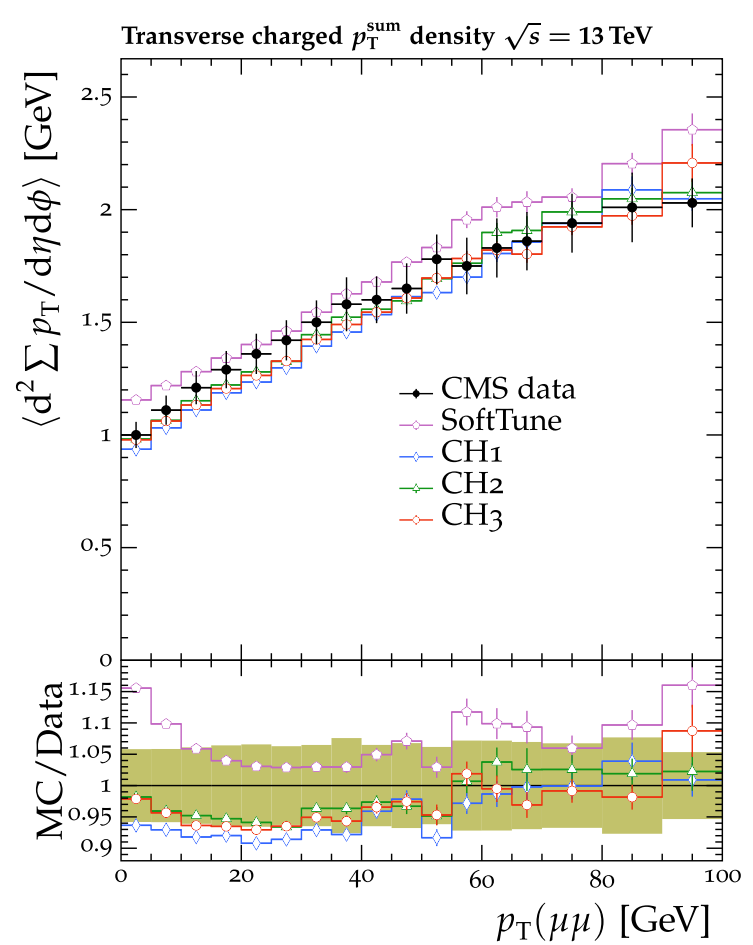

Fig. 16 The $p_{\mathrm{T}}^{\text {sum }}$ (left) and $N_{\text {ch }}$ (right) density distributions in the transverse region, as a function of the $p_{\mathrm{T}}$ of the two muons, $p_{\mathrm{T}}(\mu \mu)$ [45]. The transverse region is defined with respect to $p_{\mathrm{T}}(\mu \mu)$, where the two muons are required to have an invariant mass close the the mass of the $\mathrm{Z}$ boson. CMS Z boson data are compared with the predictions

and sphericity $(S)$ observables as measured by the ALEPH Collaboration [32].

Because these observables are measured in collisions with a lepton-lepton initial state, the difference in choice of PDF and parameters of the MPI model in the three $\mathrm{CH}$ tunes has no effect on the predictions. Similarly, the only difference between the $\mathrm{CH}$ tunes and SoftTune is in the value of $\alpha_{\mathrm{S}}\left(m_{\mathrm{Z}}\right)$. The value of $\alpha_{\mathrm{S}}\left(m_{\mathrm{Z}}\right)=0.118$ is used in the $\mathrm{CH}$ tunes, and is consistent with the value used by the PDF set for the hard process and the PS when simulating proton-proton collisions. A set of next-to-leading corrections to soft gluon emissions can be incorporated in the PS by using two-loop running of $\alpha_{\mathrm{S}}$ and including the Catani-Marchesini-Webber rescaling [33] of $\alpha_{\mathrm{S}}\left(m_{\mathrm{Z}}\right)$ from $\alpha_{\mathrm{S}}\left(m_{\mathrm{Z}}\right)=0.118$ to $\alpha_{\mathrm{S}}\left(m_{\mathrm{Z}}\right)=0.1262$, which corresponds to the value of $\alpha_{\mathrm{S}}\left(m_{\mathrm{Z}}\right)$ used in SoftTune [34].

The $\mathrm{CH}$ tunes underestimate the number of events with $0.80<T<0.95$, whereas SoftTune predicts too many isotropic events with lower values of $T<0.8$ and with higher values of $S>0.4$. The $\mathrm{CH}$ tune provides a better overall description of the $T_{\text {major }}$ observable compared with Soft Tune. Both tunes predict too many planar events, as can be seen at larger values of $O$; however, the $\mathrm{CH}$ tune provides a better description of the data at smaller values of $O$.

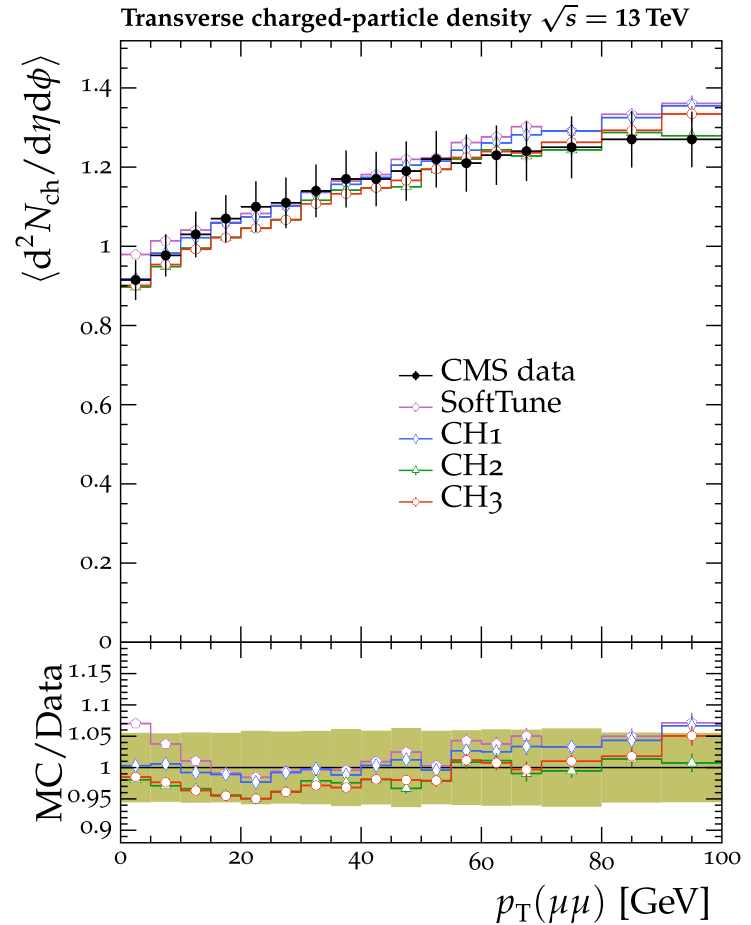

from MADGRAPH5_aMC@NLO + HERWIG 7, with the SoftTune and CH tunes. The coloured band in the ratio plot represents the total experimental uncertainty in the data. The vertical bars on the points for the different predictions represent the statistical uncertainties

\section{Comparison with top quark pair production data}

Predictions using the HERWIG 7 tunes are compared in this section with observables measured in data containing top quark pairs.

The POWHEG v2 generator is used to perform ME calculations in the hvq mode [35] at NLO accuracy in QCD. In the POWHEG ME calculations, a value of $\alpha_{\mathrm{S}}\left(m_{\mathrm{Z}}\right)=0.118$ with a two-loop evolution of $\alpha_{\mathrm{S}}$ is used, along with the NNPDF 3.1 NNLO PDF set, derived with a value of $\alpha_{\mathrm{S}}\left(m_{\mathrm{Z}}\right)=0.118$. The ME calculations are interfaced with HERWIG 7 for the simulation of the UE and PS. The mass of the top quark is set to $m_{\mathrm{t}}=172.5 \mathrm{GeV}$, and the value of the $h_{\mathrm{damp}}$ parameter, which controls the matching between the ME and PS, is set to $1.379 m_{\mathrm{t}}$. The value of $h_{\text {damp }}$ in POWHEG was derived from a fit to tit data in the dilepton channel at $\sqrt{s}=8 \mathrm{TeV}$, where POWHEG was interfaced with PYTHIA 8 using the CP5 tune $[19,36]$.

Samples are generated with the different HERWIG 7 tunes that use the same parton-level events for each tune. For generating NLO matched samples such as these, an NLO (or NNLO) PDF set may be desirable for the simulation of the hard process. In Ref. [37], it is then advocated that the same PDF set and $\alpha_{\mathrm{S}}\left(m_{\mathrm{Z}}\right)$ value should be used in the PS. However, one can still choose an LO PDF set for the simulation 

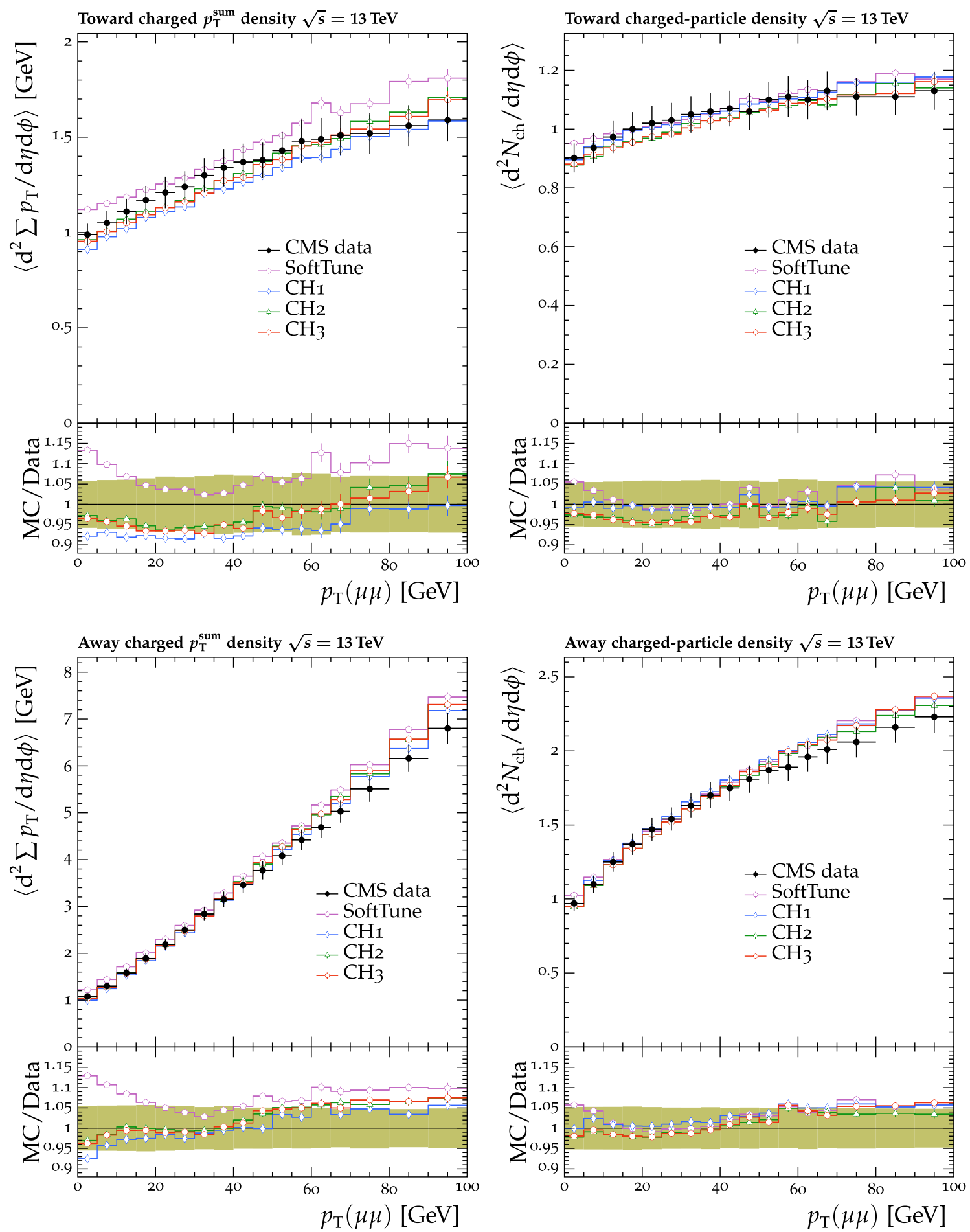

Fig. 17 The $p_{\mathrm{T}}^{\text {sum }}$ (left) and $N_{\text {ch }}$ (right) density distributions in the toward (upper), and away (lower) regions, as a function of the $p_{\mathrm{T}}$ of the two muons, $p_{\mathrm{T}}(\mu \mu)$ [45]. The toward and away regions are defined with respect to $p_{\mathrm{T}}(\mu \mu)$, where the two muons are required to have an invariant mass close the the mass of the Z boson. CMS Z boson data

of the MPI and remnant handling in this case, such as the choices in the tunes $\mathrm{CH} 2$ and $\mathrm{CH} 3$. This configuration of PDF sets is not possible in PYTHIA.

are compared with the predictions from MADGRAPH5_aMC@NLO + HERWIG 7, with the SoftTune and $\mathrm{CH}$ tunes. The coloured band in the ratio plot represents the total experimental uncertainty in the data. The vertical bars on the points for the different predictions represent the statistical uncertainties

First, kinematic properties of the $\overline{\mathrm{t}}$ system are compared with $\sqrt{s}=13 \mathrm{TeV}$ CMS data in the single-lepton channel [38]. Figure 12 presents normalized differential cross sec- 


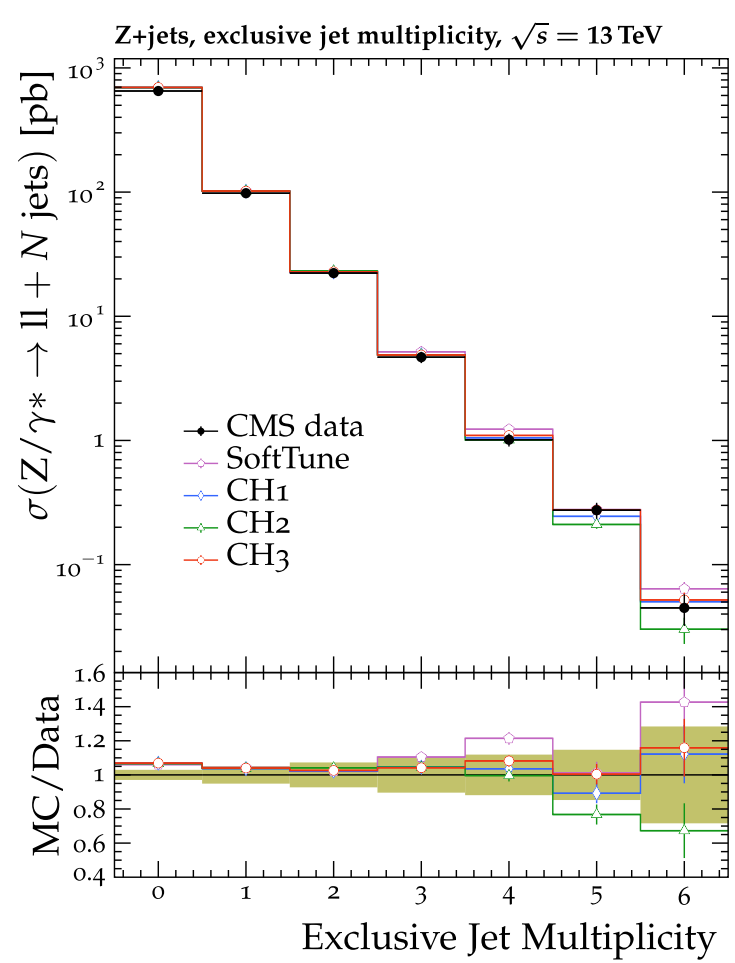

Fig. 18 The exclusive jet multiplicity in $\mathrm{Z}$ (left) and W (right) boson events, measured by CMS at $\sqrt{s}=13 \mathrm{TeV}[46,47]$. CMS Zboson and $\mathrm{W}$ boson data are compared with the predictions from MADGRAPH5_aMC@NLO + HERWIG 7, with the SoftTune and CH tunes.

tions as functions of the $p_{\mathrm{T}}$ and rapidity $y$ of the particlelevel hadronically decaying top quark. The invariant mass of the reconstructed tt system and the number of additional jets with $p_{\mathrm{T}}>30 \mathrm{GeV}$ in the event are also shown, where the jets are reconstructed using the anti- $k_{\mathrm{T}}$ algorithm $[39,40]$ with a distance parameter of 0.4 . Normalized cross sections as a function of global event variables, namely $H_{\mathrm{T}}$, the scalar $p_{\mathrm{T}}$ sum of all jets, and $p_{\mathrm{T}}^{\text {miss }}$, the magnitude of the missing transverse momentum vector [41] are shown in Fig. 13.

The predictions from the different simulations are mostly compatible with each other, indicating a small effect of the tune on these observables. The only notable difference is seen in the additional jet multiplicity, originating from the smaller $\alpha_{\mathrm{S}}\left(m_{\mathrm{Z}}\right)$ value used in the simulations with HERWIG 7 $\mathrm{CH}$ tunes. The simulated events with the $\mathrm{CH}$ tunes describe the CMS data well up to 4 additional jets, but slightly underestimate the multiplicity for a higher number of jets. The differences between the predictions with the $\mathrm{CH}$ tunes and the tune SoftTune are comparable with the typical size of the theoretical uncertainties in the ME calculation, as studied in Ref. [36].

Next, jet substructure observables are compared to $\sqrt{s}=$ $13 \mathrm{TeV}$ CMS data in the single-lepton channel [42]. Normalized number of jets as a function of four variables with relatively low correlations amongst themselves are shown in

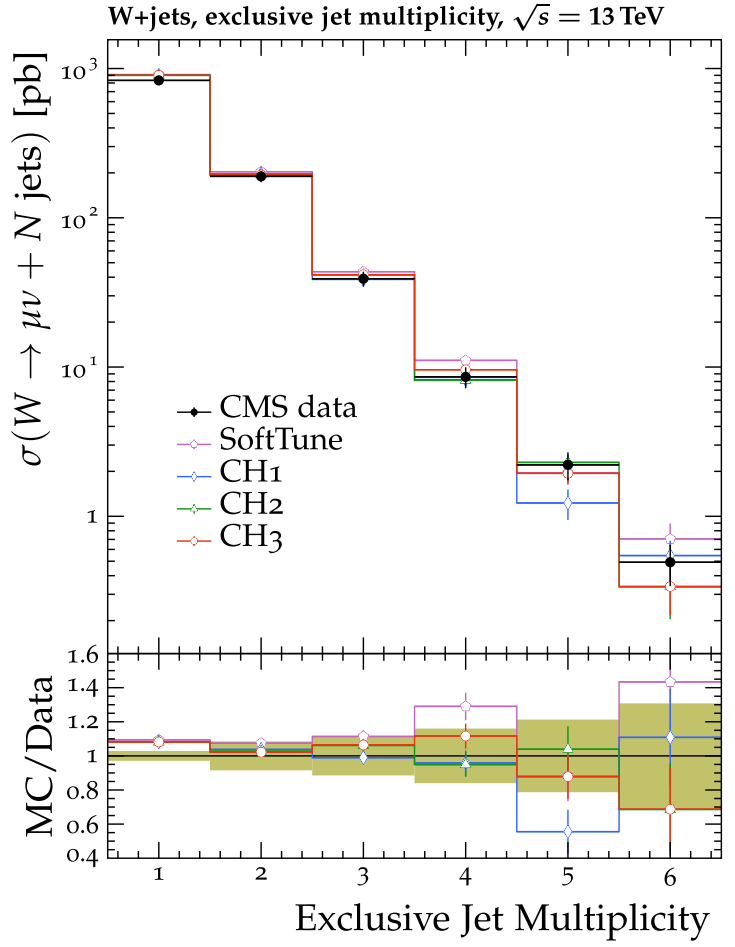

The coloured band in the ratio plot represents the total experimental uncertainty in the data. The vertical bars on the points for the different predictions represent the statistical uncertainties

Fig. 14. The variables presented are the charged-particle multiplicity $\left(\lambda_{0}^{0}\right)$, the eccentricity $(\varepsilon)$ calculated from the charged jet constituents, the groomed momentum fraction $\left(z_{\mathrm{g}}\right)$, and the angle between the groomed subjets $\left(\Delta R_{\mathrm{g}}\right)$.

The choice of tune has little effect on most of the jet substructure observables. All choices of HERWIG 7 tune overestimate $\lambda_{0}^{0}$, which was also observed in Ref. [42]. The predictions for $\varepsilon$ and $z_{\mathrm{g}}$ distributions agree closely with the data in all cases. The $\Delta R_{\mathrm{g}}$ spectrum at very low values is somewhat less well described by the simulation employing the $\mathrm{CH}$ tunes, whereas for high values the description is better for the $\mathrm{CH}$ tune samples than with SoftTune. Since the $\Delta R_{\mathrm{g}}$ observable is strongly dependent on the amount of final-state radiation [42], the difference comes mostly from the choice of $\alpha_{\mathrm{S}}\left(m_{\mathrm{Z}}\right)$, with the choice of $\alpha_{\mathrm{S}}\left(m_{\mathrm{Z}}\right)$ in the CH tunes preferred to that in SoftTune.

\section{Comparisons with inclusive jet events}

The predictions of HERWIG 7 with the various tunes for inclusive jet production are investigated in this section. In particular, the substructure of the jets is considered. Events are generated with the LO QCD two-to-two MEs implemented in HERWIG 7. Although a comparison of the substructure of 

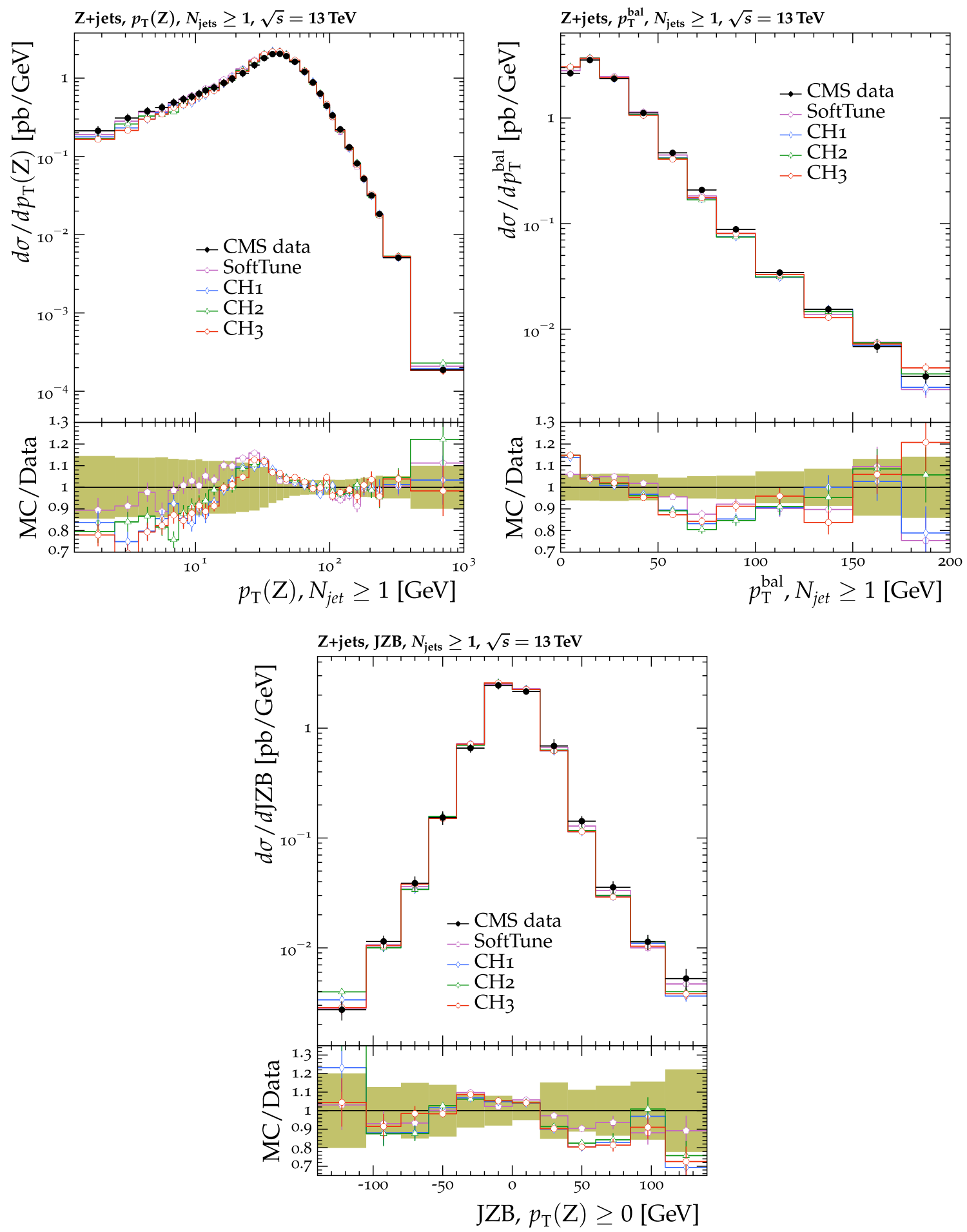

Fig. 19 Differential cross sections as a function of $p_{\mathrm{T}}(\mathrm{Z})$ (upper left), $p_{\mathrm{T}}^{\text {bal }}$ (upper right), and JZB (lower) [46]. CMS Zboson data are compared with the predictions from MADGRAPH5_aMC@NLO + HERWIG 7, with the SoftTune and $\mathrm{CH}$ tunes. The coloured band in the ratio plot

jets in ț events was already presented in Sect. 7, the comparison based on inclusive jet events is complementary because it probes a wider range of jet $p_{\mathrm{T}}$. represents the total experimental uncertainty in the data. The vertical bars on the points for the different predictions represent the statistical uncertainties

Figure 15 shows the differential jet shape, $\rho(\mathrm{r})$, as measured by the CMS experiment at $\sqrt{s}=7 \mathrm{TeV}$ [43] for two bins of ranges of jet $p_{\mathrm{T}}\left(p_{\mathrm{T}}^{\text {jet }}\right): 40<p_{\mathrm{T}}^{\text {jet }}<50 \mathrm{GeV}$ and 
$600<p_{\mathrm{T}}^{\text {jet }}<1000 \mathrm{GeV}$. The observable $\rho(\mathrm{r})$ is defined as the average fraction of the $p_{\mathrm{T}}$ of the jet constituents contained inside an annulus with inner radius $r-0.1$ and outer radius $r+0.1$. The second moment of the jet transverse width, $\left\langle\delta R^{2}\right\rangle$, is also shown. The jets are clustered with the anti- $k_{\mathrm{T}}$ algorithm with a distance parameter of 0.7 for the jet shape observables, and 0.5 for the $\left\langle\delta R^{2}\right\rangle$ observable. The predictions from the three $\mathrm{CH}$ tunes are very similar for all distributions, and agree with the data. On the other hand, the prediction from SoftTune differs from the $\mathrm{CH}$ tunes, and also does not agree well with the $\left\langle\delta R^{2}\right\rangle$ distribution in data.

Additional comparisons of the predictions for various tunes of HERWIG 7 tunes with the substructure of jets collected by the ATLAS experiment are shown in Appendix B.

\section{Comparison with $\mathrm{Z}$ and $\mathrm{W}$ boson production data}

In this section, the performance of the HERWIG 7 tunes is compared with $\sqrt{s}=13 \mathrm{TeV}$ data on $\mathrm{Z}$ and $\mathrm{W}$ boson production. Predictions for $\mathrm{Z}$ and $\mathrm{W}$ boson production are obtained with MADGRAPH5_aMC@NLO v2.6.7 [9] for ME calculations at NLO, which are interfaced with HERWIG 7 using the the FxFx merging scheme [44], with the merging scale set to $30 \mathrm{GeV}$. Up to two additional partons in the final state are included in the NLO ME calculations. The PDF in the ME calculations is NNPDF3.1 NNLO, and the value of $\alpha_{\mathrm{S}}\left(m_{\mathrm{Z}}\right)$ in the ME calculations is set to $\alpha_{\mathrm{S}}\left(m_{\mathrm{Z}}\right)=0.118$ in all the predictions considered here.

First, the $p_{\mathrm{T}}^{\text {sum }}$ and $N_{\mathrm{ch}}$ distributions characterizing the UE in Z boson production [45] are compared to simulation in Figs. 16 and 17. Events are required to have two muons with an invariant mass between 81 and $101 \mathrm{GeV}$ to select events within the $\mathrm{Z}$ boson mass peak. The $p_{\mathrm{T}}^{\text {sum }}$ and $N_{\text {ch }}$ distributions are measured in the transverse region as shown in Fig. 16, and in the toward and away regions as shown in Fig. 17, in analogy to the corresponding distributions measured in MB data introduced in Sect. 3. The regions are defined with respect to the $p_{\mathrm{T}}$ of the $Z$ boson, calculated from the $p_{\mathrm{T}}$ of the two muons. The $\mathrm{CH}$ tunes describe the data well, and are typically similar to each other. However, the configuration with SoftTune fails to give a simultaneous description of the $p_{\mathrm{T}}^{\text {sum }}$ and $N_{\mathrm{ch}}$ distributions in any region at low $p_{\mathrm{T}}(\mu \mu)$.

Next, the exclusive jet multiplicity distributions in Z and W boson events are shown in Fig. 18 [46,47]. Events in the $\mathrm{Z}$ boson sample contain at least two electrons or muons with $p_{\mathrm{T}}>20 \mathrm{GeV}$ and $|\eta|<2.4$, and the invariant mass of the two highest $p_{\mathrm{T}}$ electrons or muons must have an invariant mass within $20 \mathrm{GeV}$ of the $\mathrm{Z}$ boson mass. In the $\mathrm{W}$ boson measurement, only final states with a muon of $p_{\mathrm{T}}>25 \mathrm{GeV}$ and $|\eta|<$ 2.4 are considered. The transverse mass of the $\mathrm{W}$ boson candidate, defined as $m_{\mathrm{T}}=\sqrt{2 p_{\mathrm{T}}^{\mu} p_{\mathrm{T}}^{\text {miss }}\left[1-\cos \left(\Delta \phi_{\mu, \vec{p}_{\mathrm{T}}^{\text {miss }}}\right)\right]}$, where $\cos \left(\Delta \phi_{\mu, \vec{p}_{\mathrm{T}}^{\text {miss }}}\right)$ is the difference in azimuthal angle between the direction of the muon momentum and $p_{\mathrm{T}}^{\text {miss }}$, must satisfy $m_{\mathrm{T}}>50 \mathrm{GeV}$. In both $\mathrm{Z}$ and $\mathrm{W}$ events jets are reconstructed using the anti- $k_{\mathrm{T}}$ algorithm with a distance parameter of 0.4 , and are required to satisfy $p_{\mathrm{T}}>30 \mathrm{GeV}$ and $|y|<2.4$. Jets must also be separated from any lepton by $\sqrt{(\Delta \eta)^{2}+(\Delta \phi)^{2}}>0.4$, where $\phi$ is in radians. The jet multiplicity is well described by all tunes in both $\mathrm{Z}$ and W boson events at both low multiplicities, where the ME calculations dominate, and high multiplicities, where the PS is important.

Finally, in Fig. 19, the $p_{\mathrm{T}}(\mathrm{Z})$ and $p_{\mathrm{T}}^{\text {bal }}$ distributions are shown, both for final states containing at least one additional jet. The $p_{\mathrm{T}}^{\text {bal }}$ variable is defined as $p_{\mathrm{T}}^{\text {bal }}=\mid \vec{p}_{\mathrm{T}}(\mathrm{Z})+$ $\sum_{\text {jets }} \vec{p}_{\mathrm{T}}(\mathrm{j}) \mid$. The so-called jet-Zbalance (JZB) variable, defined as JZB $=\left|\sum_{\text {jets }} \vec{p}_{\mathrm{T}}(\mathrm{j})\right|-\left|\vec{p}_{\mathrm{T}}(\mathrm{Z})\right|$, is also shown in Fig. 19. All distributions are measured for events with at least one additional jet. The $p_{\mathrm{T}}(\mathrm{Z})$ predictions for all tunes are similar for $p_{\mathrm{T}}(\mathrm{Z})>30 \mathrm{GeV}$, where the predictions are driven by the ME calculations. At lower $p_{\mathrm{T}}(\mathrm{Z})$, where events contain additional hadronic activity that is not clustered into jets, the predictions with the $\mathrm{CH}$ tunes are similar to each other, and differ slightly from the prediction with SoftTune, which provides a closer description of the data at very low $p_{\mathrm{T}}(\mathrm{Z})<10 \mathrm{GeV}$. The $p_{\mathrm{T}}^{\mathrm{bal}}$ and JZB distributions are also sensitive to additional hadronic activity not clustered into jets. For $p_{\mathrm{T}}^{\text {bal }}$, all tunes are compatible with each other, except at $p_{\mathrm{T}}^{\text {bal }}<10 \mathrm{GeV}$, where the prediction with SoftTune differs from the predictions with the $\mathrm{CH}$ tunes. The JZB distributions are well described by all the predictions.

\section{Summary}

Three new tunes for the multiple-parton interaction (MPI) model of the HERWIG 7 (version 7.1.4) generator have been derived from minimum-bias (MB) data collected by the CMS experiment. All of the $\mathrm{CH}$ ("CMS HERWIG") tunes, $\mathrm{CH} 1$, $\mathrm{CH} 2$, and $\mathrm{CH} 3$, are based on the next-to-next-to-leadingorder (NNLO) NNPDF 3.1 PDF set for the simulation of the parton shower (PS) in HERWIG 7; the value of the strong coupling at a scale equal to the $\mathrm{Z}$ boson mass is $\alpha_{\mathrm{S}}\left(m_{\mathrm{Z}}\right)=0.118$ with a two-loop evolution of $\alpha_{\mathrm{S}}$. The configuration of the tunes differs in the PDF used for the simulation of MPI and beam remnants. The tune $\mathrm{CH} 1$ uses the same NNLO PDF set for these aspects of the HERWIG 7 simulation, whereas $\mathrm{CH} 2$ and $\mathrm{CH} 3$ use leading-order (LO) versions of the PDF set. The tune $\mathrm{CH} 2$ is based on an LO PDF set that was derived assuming $\alpha_{\mathrm{S}}\left(m_{\mathrm{Z}}\right)=0.118$, and $\mathrm{CH} 3$ on an LO PDF set assuming $\alpha_{\mathrm{S}}\left(m_{\mathrm{Z}}\right)=0.130$.

The parameters of the MPI model were optimized for each tune with the PROFESSOR framework to describe the under- 
lying event (UE) in MB data collected by CMS. The predictions using the tune $\mathrm{CH} 2$ or $\mathrm{CH} 3$ provide a better description of the data than those using $\mathrm{CH} 1$ or SoftTune. Furthermore, the differences in the predictions of $\mathrm{CH} 2$ and $\mathrm{CH} 3$ are observed to be small. The configuration of PDF sets in the tune $\mathrm{CH} 3$, where the LO PDF used for the simulation of MPI, was derived with a value of $\alpha_{\mathrm{S}}\left(m_{\mathrm{Z}}\right)$ typically associated with LO PDF sets, is the preferred choice over $\mathrm{CH} 2$. Two alternative tunes representing the uncertainties in the fitted parameters of $\mathrm{CH} 3$ are also derived, based on the tuning procedure provided by PROFESSOR.

Predictions using the three $\mathrm{CH}$ tunes are compared with a range of data beyond MB events: event shape data from LEP; proton-proton data enriched in top quark pairs, $\mathrm{Z}$ bosons and W bosons; and inclusive jet data. This validated the performance of HERWIG 7 using these tunes against a wide range of data sets sensitive to various aspects of the modelling by HERWIG 7, and in particular the modelling of the UE. The event shape observables measured at LEP, which are sensitive to the modelling of final-state radiation, are well described by HERWIG 7 with the new tunes. Predictions using the new tunes are also shown to describe the UE in events containing $\mathrm{Z}$ bosons, demonstrating the universality of the UE modelling in HERWIG 7. The kinematics of top quark events, and the modelling of jets in $\bar{t} \overline{\mathrm{t}}, \mathrm{Z}$ boson, $\mathrm{W}$ boson, and inclusive jet data are also well described by predictions using the new tunes. In general, predictions with the new $\mathrm{CH}$ tunes derived in this paper provide a better description of measured observables than those using SoftTune, the default tune available in HERWIG 7.

Acknowledgements We congratulate our colleagues in the CERN accelerator departments for the excellent performance of the LHC and thank the technical and administrative staffs at CERN and at other CMS institutes for their contributions to the success of the CMS effort. In addition, we gratefully acknowledge the computing centres and personnel of the Worldwide LHC Computing Grid for delivering so effectively the computing infrastructure essential to our analyses. Finally, we acknowledge the enduring support for the construction and operation of the LHC and the CMS detector provided by the following funding agencies: BMBWF and FWF (Austria); FNRS and FWO (Belgium); CNPq, CAPES, FAPERJ, FAPERGS, and FAPESP (Brazil); MES (Bulgaria); CERN; CAS, MoST, and NSFC (China); COLCIENCIAS (Colombia); MSES and CSF (Croatia); RIF (Cyprus); SENESCYT (Ecuador); MoER, ERC PUT and ERDF (Estonia); Academy of Finland, MEC, and HIP (Finland); CEA and CNRS/IN2P3 (France); BMBF, DFG, and HGF (Germany); GSRT (Greece); NKFIA (Hungary); DAE and DST (India); IPM (Iran); SFI (Ireland); INFN (Italy); MSIP and NRF (Republic of Korea); MES (Latvia); LAS (Lithuania); MOE and UM (Malaysia); BUAP, CINVESTAV, CONACYT, LNS, SEP, and UASLPFAI (Mexico); MOS (Montenegro); MBIE (New Zealand); PAEC (Pakistan); MSHE and NSC (Poland); FCT (Portugal); JINR (Dubna); MON, RosAtom, RAS, RFBR, and NRC KI (Russia); MESTD (Serbia); SEIDI, CPAN, PCTI, and FEDER (Spain); MOSTR (Sri Lanka); Swiss Funding Agencies (Switzerland); MST (Taipei); ThEPCenter, IPST, STAR, and NSTDA (Thailand); TUBITAK and TAEK (Turkey); NASU (Ukraine); STFC (United Kingdom); DOE and NSF (USA). Individuals have received support from the Marie-Curie programme and the European Research Council and Horizon 2020 Grant, contract Nos. 675440, 724704, 752730, and 765710 (European Union); the Leventis Foundation; the A.P. Sloan Foundation; the Alexander von Humboldt Foundation; the Belgian Federal Science Policy Office; the Fonds pour la Formation à la Recherche dans l'Industrie et dans l'Agriculture (FRIA-Belgium); the Agentschap voor Innovatie door Wetenschap en Technologie (IWT-Belgium); the F.R.S.-FNRS and FWO (Belgium) under the "Excellence of Science - EOS" - be.h project $n$. 30820817; the Beijing Municipal Science \& Technology Commission, No. Z191100007219010; the Ministry of Education, Youth and Sports (MEYS) of the Czech Republic; the Deutsche Forschungsgemeinschaft (DFG) under Germany's Excellence Strategy - EXC 2121 "Quantum Universe" - 390833306; the Lendület ("Momentum") Programme and the János Bolyai Research Scholarship of the Hungarian Academy of Sciences, the New National Excellence Program ÚNKP, the NKFIA research grants 123842, 123959, 124845, 124850, 125105, 128713, 128786, and 129058 (Hungary); the Council of Science and Industrial Research, India; the HOMING PLUS programme of the Foundation for Polish Science, cofinanced from European Union, Regional Development Fund, the Mobility Plus programme of the Ministry of Science and Higher Education, the National Science Center (Poland), contracts Harmonia 2014/14/M/ST2/00428, Opus 2014/13/B/ST2/02543, 2014/15/B/ST2/03998, and 2015/19/B/ST2/02861, Sonata-bis 2012/07/ E/ST2/01406; the National Priorities Research Program by Qatar National Research Fund; the Ministry of Science and Higher Education, project no. 02.a03.21.0005 (Russia); the Tomsk Polytechnic University Competitiveness Enhancement Program; the Programa Estatal de Fomento de la Investigación Científica y Técnica de Excelencia María de Maeztu, grant MDM-2015-0509 and the Programa Severo Ochoa del Principado de Asturias; the Thalis and Aristeia programmes cofinanced by EU-ESF and the Greek NSRF; the Rachadapisek Sompot Fund for Postdoctoral Fellowship, Chulalongkorn University and the Chulalongkorn Academic into Its 2nd Century Project Advancement Project (Thailand); the Kavli Foundation; the Nvidia Corporation; the SuperMicro Corporation; the Welch Foundation, contract C-1845; and the Weston Havens Foundation (USA).

Data Availability Statement This manuscript has no associated data or the data will not be deposited. [Authors' comment: Release and preservation of data used by the CMS Collaboration as the basis for publications is guided by the CMS policy as written in its document "CMS data preservation, re-use and open access policy" (https://cms-docdb.cern. ch/cgi-bin/PublicDocDB/RetrieveFile?docid=6032\&filename=CMSD ataPolicyV1.2.pdf\&version=2).]

\section{Compliance with ethical standards}

Conflict of interest The authors declare that they have no conflict of interest.

Open Access This article is licensed under a Creative Commons Attribution 4.0 International License, which permits use, sharing, adaptation, distribution and reproduction in any medium or format, as long as you give appropriate credit to the original author(s) and the source, provide a link to the Creative Commons licence, and indicate if changes were made. The images or other third party material in this article are included in the article's Creative Commons licence, unless indicated otherwise in a credit line to the material. If material is not included in the article's Creative Commons licence and your intended use is not permitted by statutory regulation or exceeds the permitted use, you will need to obtain permission directly from the copyright holder. To view a copy of this licence, visit http://creativecomm ons.org/licenses/by/4.0/.

Funded by SCOAP ${ }^{3}$. 


\section{Appendix A: Comparison with ATLAS MB data}

Figures 20, 21, 22, 23, 24 and 25 show comparisons of the tune predictions with MB data collected by the ATLAS experiment at $\sqrt{s}=0.9,7$, and $13 \mathrm{TeV}$, which were used in deriving the parameters of SoftTune. Figures 20 and 21 show the pseudorapidity distributions of charged particles at $\sqrt{s}=0.9$ and $7 \mathrm{TeV}$ respectively, for various minimum $N_{\mathrm{ch}}$. Figures 22 and 23 show the charged-particle $p_{\mathrm{T}}$ distributions at $\sqrt{s}=0.9$ and $7 \mathrm{TeV}$ respectively, for various minimum
$N_{\mathrm{ch}}$. The distributions of mean charged-particle $p_{\mathrm{T}}$ as a function of the charged-particle multiplicity are also shown in Figs. 22 and 23. Figures 24 and 25 show the pseudorapidity and charged-particle $p_{\mathrm{T}}$ distributions at $\sqrt{s}=13 \mathrm{TeV}$, for $|\eta|<2.5$ and $|\eta|<0.8$ respectively. The corresponding distributions of the mean charged-particle $p_{\mathrm{T}}$ as a function of the charged-particle multiplicity are also shown in Figs. 24 and 25 .
Fig. 20 Normalized plots [17] for the pseudorapidity of charged particles for $N_{\mathrm{ch}} \geq 1$ (upper left), and $N_{\mathrm{ch}} \geq 6$ (lower left), for charged particles with $p_{\mathrm{T}}>500 \mathrm{MeV}$. The figure on the upper right shows a similar distribution for $N_{\mathrm{ch}} \geq 2$, and the lower right for $N_{\text {ch }} \geq 20$, where the charged particles have $p_{\mathrm{T}}>100 \mathrm{MeV}$. ATLAS MB data are compared with the predictions from HERWIG 7, with the SoftTune and $\mathrm{CH}$ tunes. The coloured band in the ratio plot represents the total experimental uncertainty in the data. The vertical bars on the points for the different predictions represent the statistical uncertainties
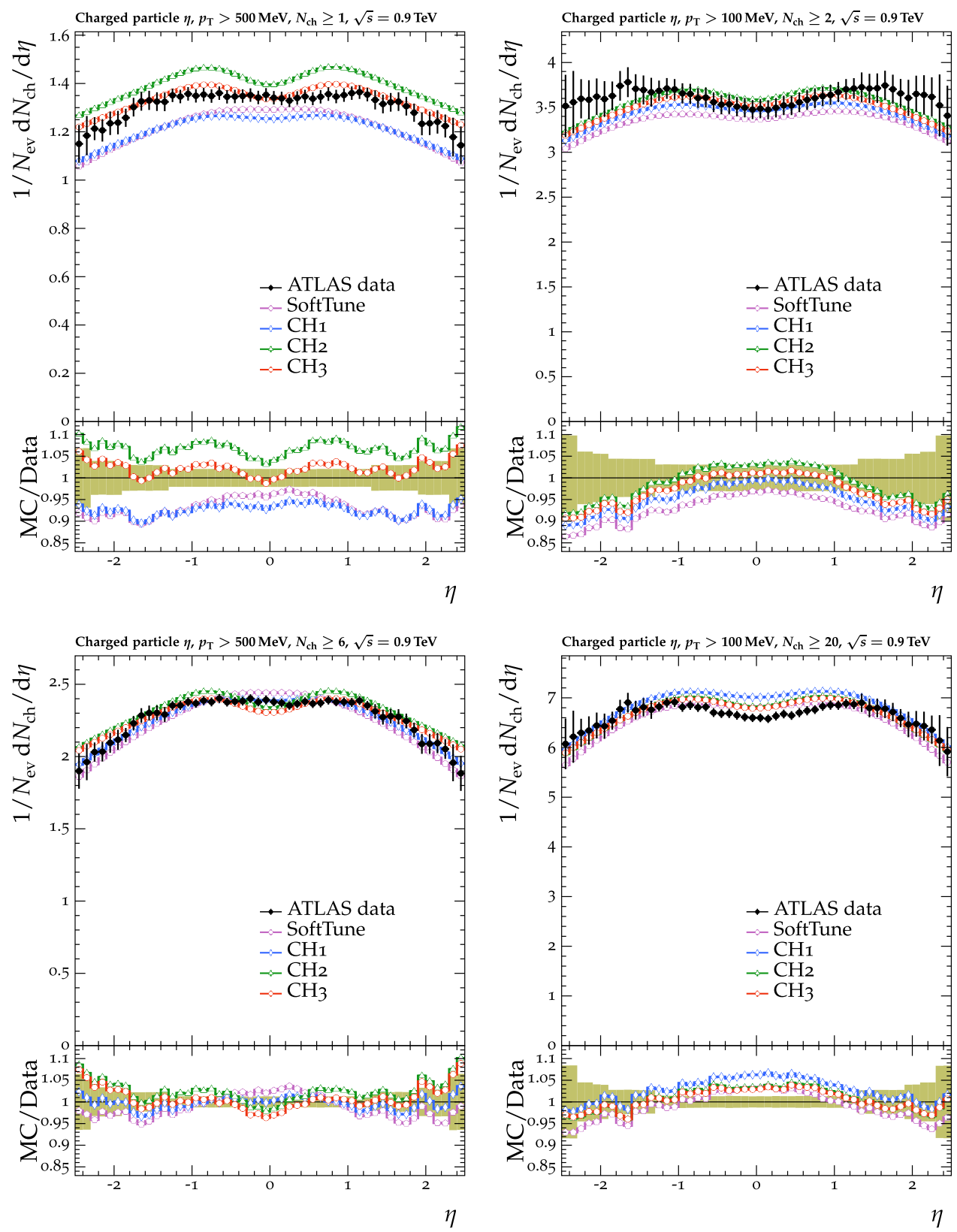

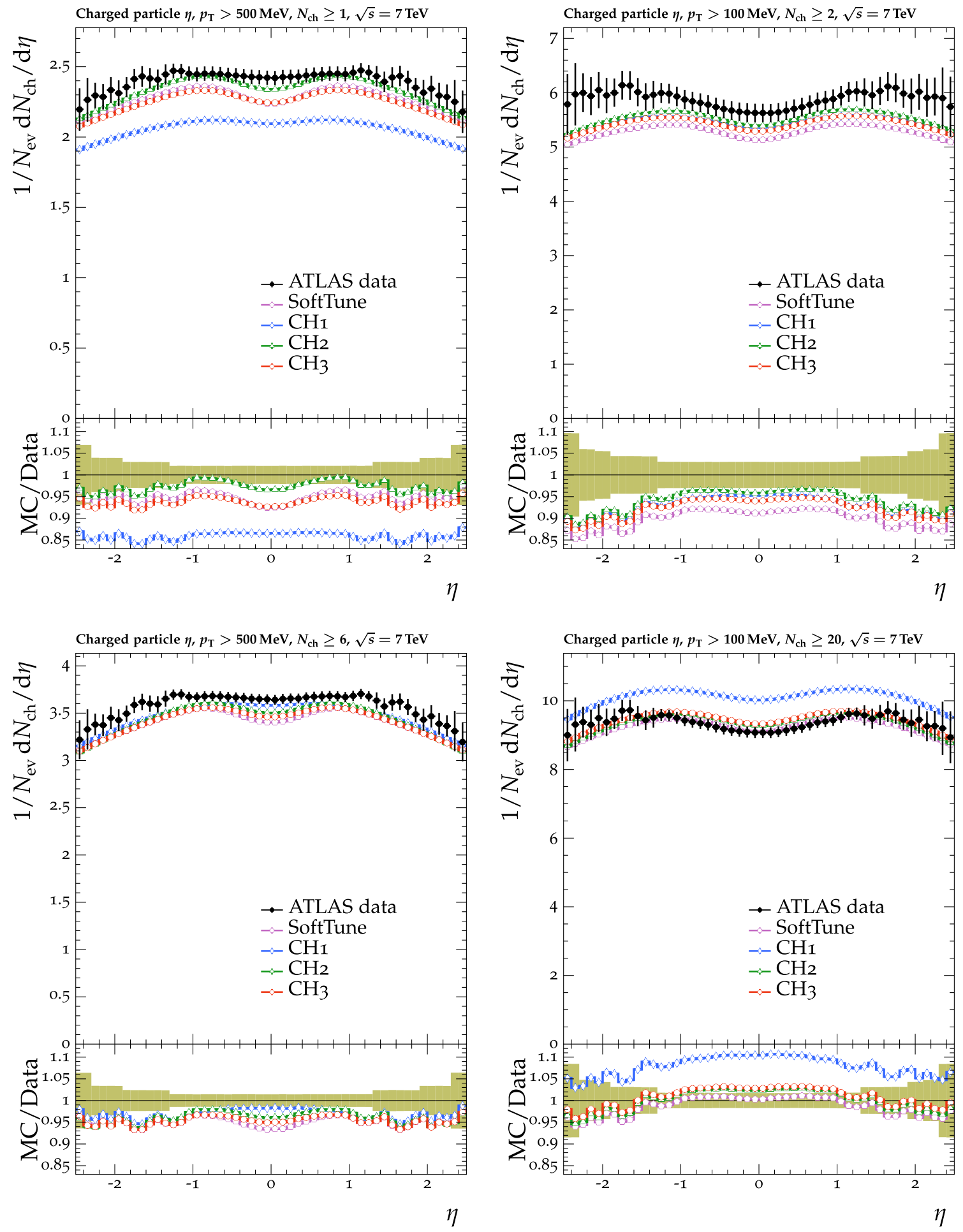

Fig. 21 Normalized plots [17] for the pseudorapidity of charged particles for $N_{\mathrm{ch}} \geq 1$ (upper left), and $N_{\mathrm{ch}} \geq 6$ (lower left), for charged particles with $p_{\mathrm{T}}>500 \mathrm{MeV}$. The figure on the upper right shows a similar distribution for $N_{\mathrm{ch}} \geq 2$, and the lower right for $N_{\mathrm{ch}} \geq 20$, where the charged particles have $p_{\mathrm{T}}>100 \mathrm{MeV}$. ATLAS MB data

are compared with the predictions from HERWIG 7, with the SoftTune and $\mathrm{CH}$ tunes. The coloured band in the ratio plot represents the total experimental uncertainty in the data. The vertical bars on the points for the different predictions represent the statistical uncertainties 

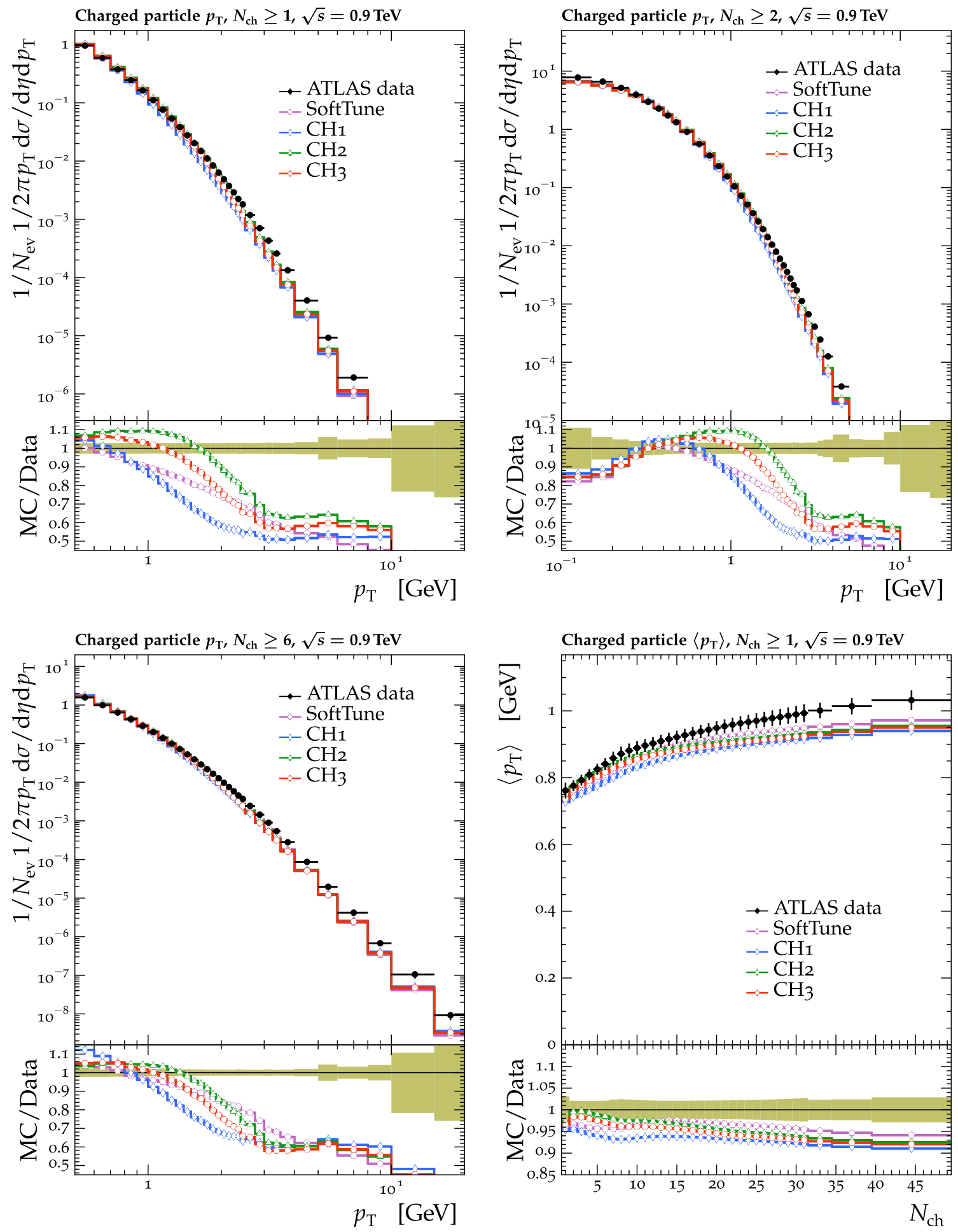

Fig. 22 Normalized plots [17] for the charged-particle $p_{\mathrm{T}}$ for $N_{\mathrm{ch}} \geq 1$ (upper left), $N_{\mathrm{ch}} \geq 2$ (upper right), and $N_{\mathrm{ch}} \geq 6$ (lower left). The mean charged-particle $p_{\mathrm{T}}$ as a function of the charged-particle multiplicity is also shown (lower right). ATLAS MB data are compared with the pre-

dictions from HERWIG 7, with the SoftTune and $\mathrm{CH}$ tunes. The coloured band in the ratio plot represents the total experimental uncertainty in the data. The vertical bars on the points for the different predictions represent the statistical uncertainties 

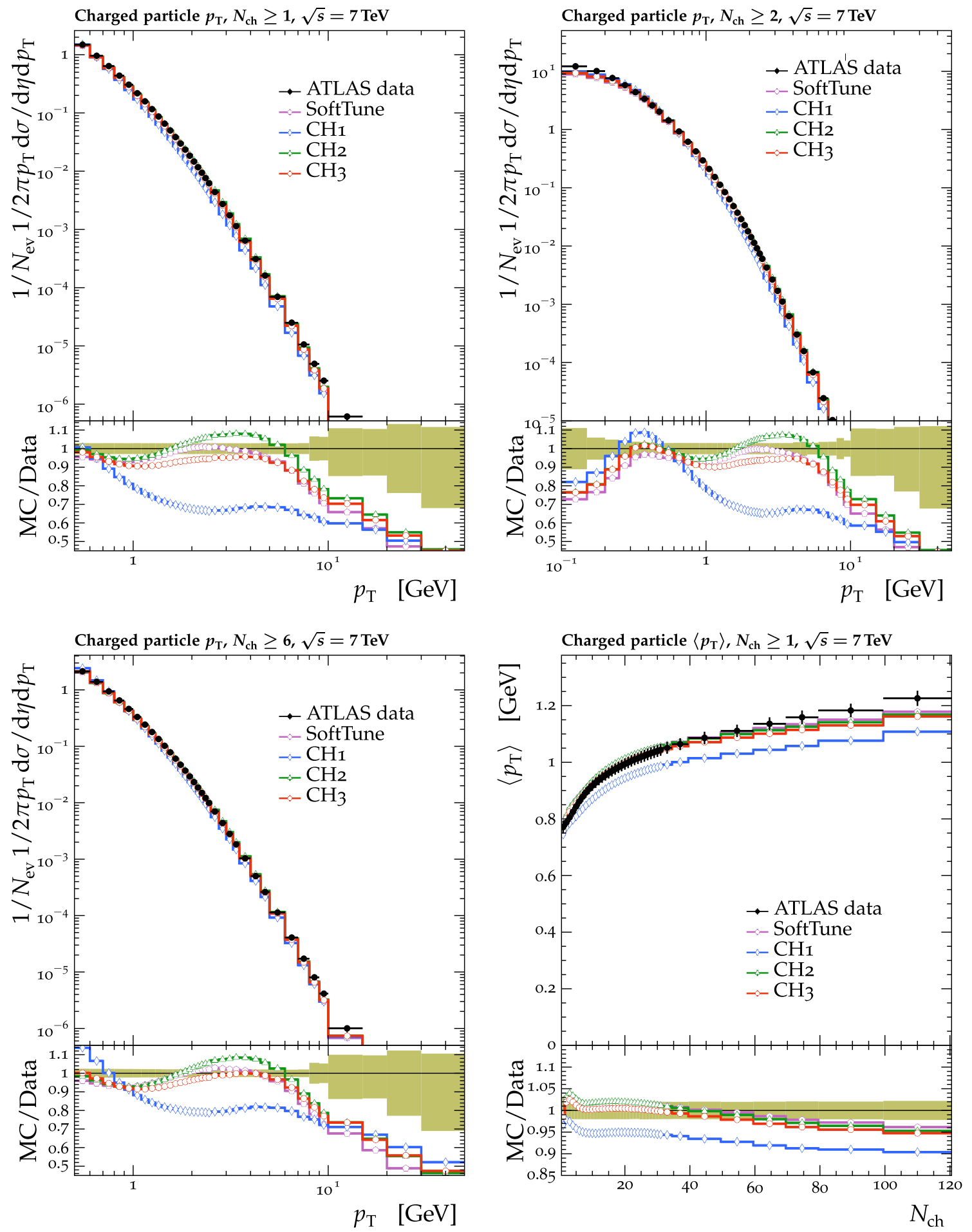

Fig. 23 Normalized plots [17] for the charged-particle $p_{\mathrm{T}}$ for $N_{\mathrm{ch}} \geq 1$ (upper left), $N_{\mathrm{ch}} \geq 2$ (upper right), and $N_{\mathrm{ch}} \geq 6$ (lower left). The mean charged-particle $p_{\mathrm{T}}$ as a function of the charged-particle multiplicity is also shown (lower right). ATLAS MB data are compared with the pre-

dictions from HERWIG 7, with the SoftTune and $\mathrm{CH}$ tunes. The coloured band in the ratio plot represents the total experimental uncertainty in the data. The vertical bars on the points for the different predictions represent the statistical uncertainties 

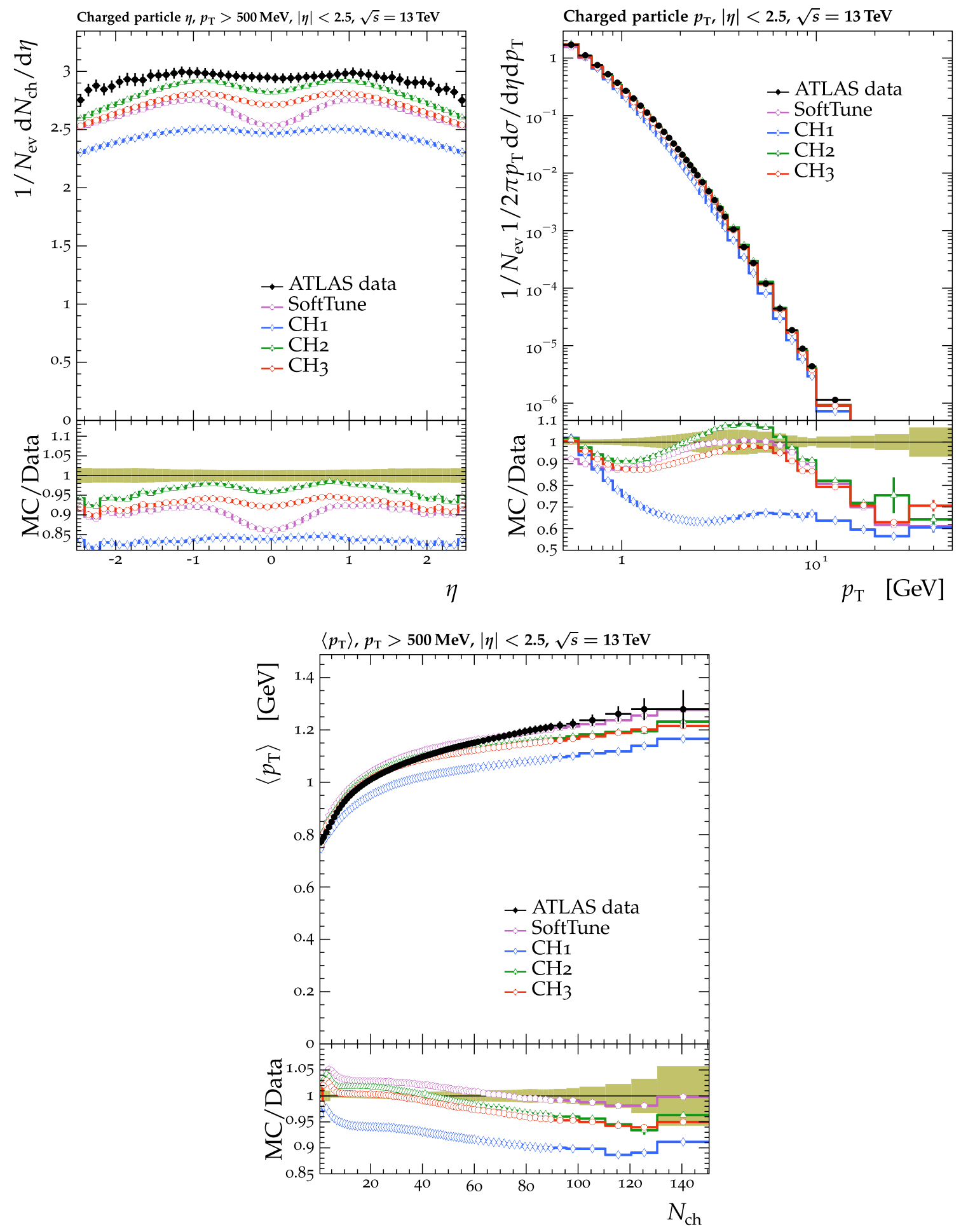

Fig. 24 Normalized plots [48] for the pseudorapidity of charged particles (upper left), charged-particle $p_{\mathrm{T}}$ distribution (upper left), and the mean charged-particle $p_{\mathrm{T}}$ distribution as a function of the chargedparticle multiplicity (lower), all for $|\eta|<2.5$. ATLAS MB data are compared with the predictions from HERWIG 7, with the SoftTune and $\mathrm{CH}$ tunes. The coloured band in the ratio plot represents the total experimental uncertainty in the data. The vertical bars on the points for the different predictions represent the statistical uncertainties 

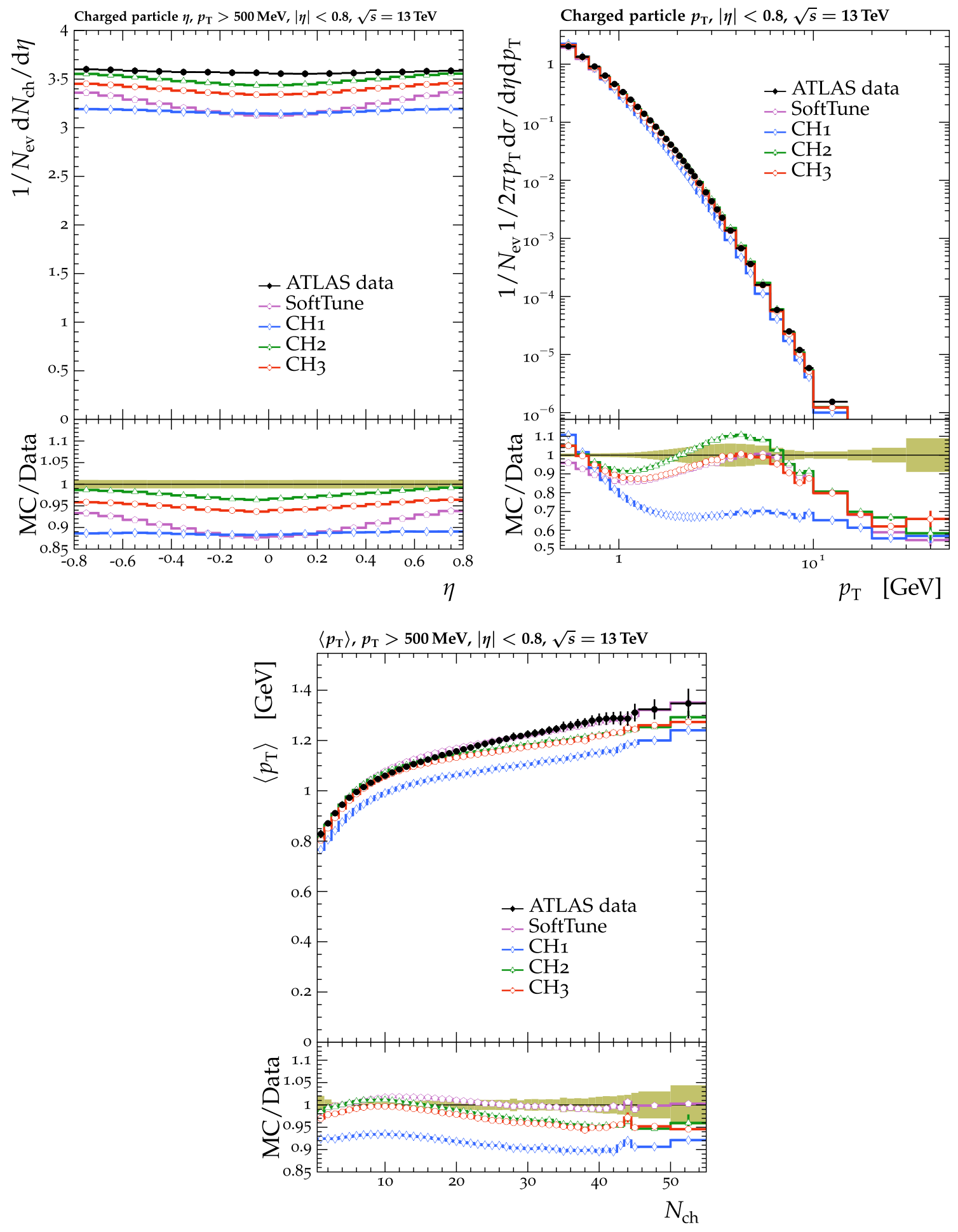

Fig. 25 Normalized plots [48] for the pseudorapidity of charged particles (upper left), charged-particle $p_{\mathrm{T}}$ distribution (upper left), and the mean charged-particle $p_{\mathrm{T}}$ distribution as a function of the chargedparticle multiplicity (lower), all for $|\eta|<0.8$. ATLAS MB data are compared with the predictions from HERWIG 7, with the SoftTune and $\mathrm{CH}$ tunes. The coloured band in the ratio plot represents the total experimental uncertainty in the data. The vertical bars on the points for the different predictions represent the statistical uncertainties 

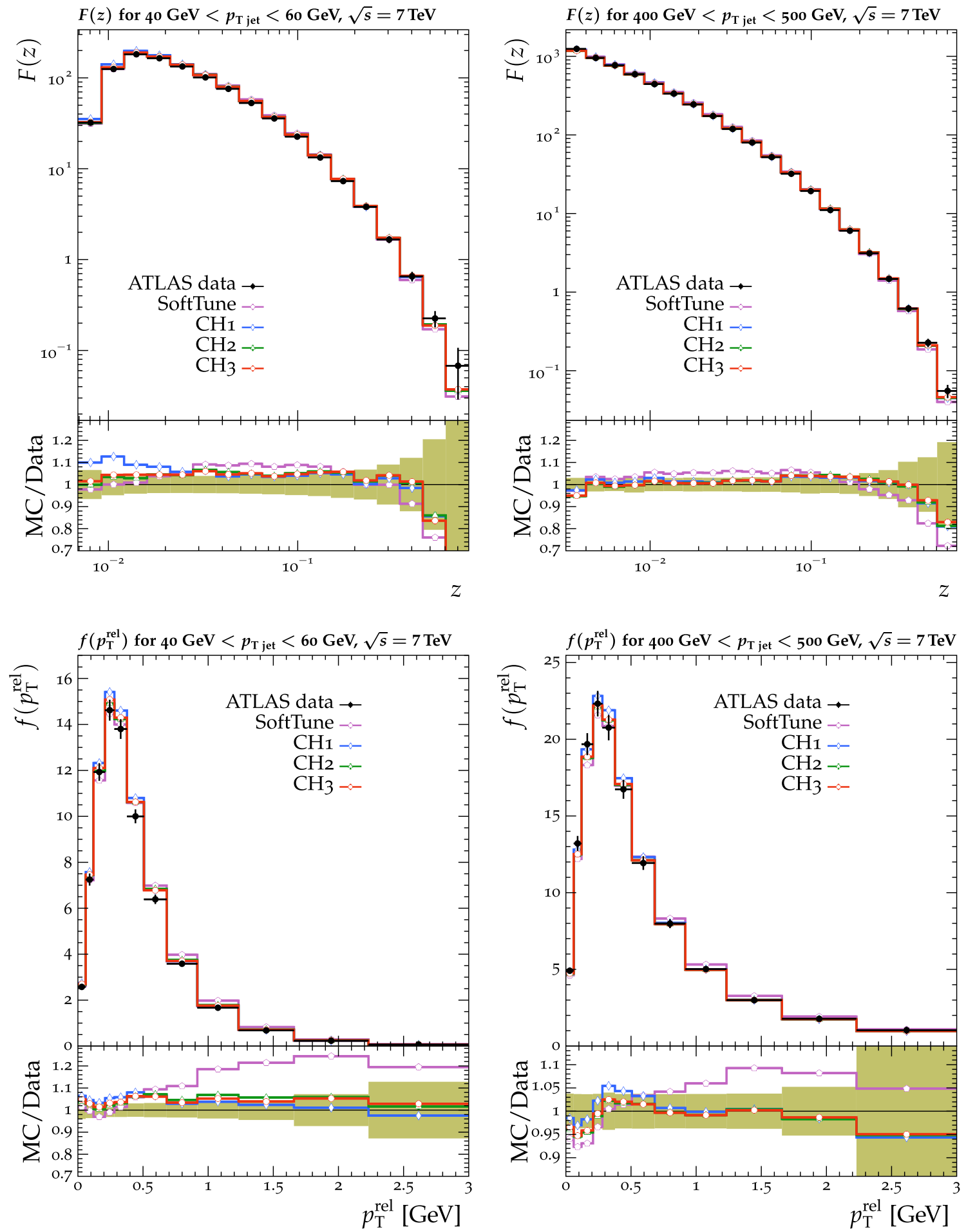

Fig. 26 The ATLAS data at $\sqrt{s}=7 \mathrm{TeV}$ on the $F(z)$ and $f\left(p_{\mathrm{T}}^{\mathrm{rel}}\right)$ distributions [17]. ATLAS inclusive jet data are compared with the predictions from HERWIG 7, with the SoftTune and $\mathrm{CH}$ tunes. The coloured

band in the ratio plot represents the total experimental uncertainty in the data. The vertical bars on the points for the different predictions represent the statistical uncertainties

\section{Appendix B: Comparison with ATLAS inclusive jet events}

Figure 26 shows the $F(z)$ distribution as a function of $z$, and the $f\left(p_{\mathrm{T}}^{\mathrm{rel}}\right)$ distribution as a function of $p_{\mathrm{T}}^{\mathrm{rel}}$, as measured by

the ATLAS experiment, along with the HERWIG 7 predictions. The former distribution is a differential measurement of the charged-particle multiplicity inside jets as a function of the fraction of the jet longitudinal momentum carried by the jet 
constituents, $z$. The latter distribution is the same data but as a function of the transverse momentum of the jet constituents, $p_{\mathrm{T}}^{\mathrm{rel}}$, with respect to the jet axis. The jets are clustered using the anti- $k_{\mathrm{T}}$ algorithm with a distance parameter of 0.6 , and the distributions are shown for two ranges of jet $p_{\mathrm{T}}\left(p_{\mathrm{T}}^{\mathrm{jet}}\right)$ : $40<p_{\mathrm{T}}^{\text {jet }}<60 \mathrm{GeV}$ and $400<p_{\mathrm{T}}^{\text {jet }}<500 \mathrm{GeV}$. For all distributions, SoftTune provides the least consistent prediction of the data. At low jet $p_{\mathrm{T}}$, the $\mathrm{CH} 2$ and $\mathrm{CH} 3$ tunes provide the best description of the data, whereas the $\mathrm{CH} 1$ tune deviates somewhat from the data both at low $z$ and at low $f\left(p_{\mathrm{T}}^{\mathrm{rel}}\right)$. At high jet $p_{\mathrm{T}}$, only SoftTune shows significant differences with respect to the data; however, these differences are smaller than those observed at low jet $p_{\mathrm{T}}$.

\section{References}

1. M. Bahr et al., HERWIG++ physics and manual. Eur. Phys. J. C 58, 639 (2008). https://doi.org/10.1140/epjc/s10052-008-0798-9. arXiv:0803.0883

2. J. Bellm et al., HERWIG 7.0/HERWIG++ 3.0 release note. Eur. Phys. J. C 76, 196 (2016). https://doi.org/10.1140/epjc/ s10052-016-4018-8. arXiv:1512.01178

3. J. Bellm et al., Herwig 7.1 release note (2017). arXiv:1705.06919

4. T. Sjöstrand et al., An introduction to PYTHIA 8.2. Comput. Phys. Commun. 191, 159 (2015). https://doi.org/10.1016/j.cpc.2015.01. 024. arXiv: 1410.3012

5. S. Platzer, S. Gieseke, Dipole showers and automated NLO matching in HERWIG++. Eur. Phys. J. C 72, 2187 (2012). https://doi. org/10.1140/epjc/s10052-012-2187-7. arXiv:1109.6256

6. P. Nason, A new method for combining NLO QCD with shower Monte Carlo algorithms. JHEP 11, 040 (2004). https://doi.org/10. 1088/1126-6708/2004/11/040. arXiv:hep-ph/0409146

7. S. Frixione, P. Nason, C. Oleari, Matching NLO QCD computations with parton shower simulations: the POWHEG method. JHEP 11, 070 (2007). https://doi.org/10.1088/1126-6708/2007/11/070. arXiv:0709.2092

8. S. Alioli, P. Nason, C. Oleari, E. Re, A general framework for implementing NLO calculations in shower Monte Carlo programs: the POWHEG BOX. JHEP 06, 043 (2010). https://doi.org/10.1007/ JHEP06(2010)043. arXiv:1002.2581

9. J. Alwall et al., The automated computation of tree-level and nextto-leading order differential cross sections, and their matching to parton shower simulations. JHEP 07, 079 (2014). https://doi.org/ 10.1007/JHEP07(2014)079. arXiv:1405.0301

10. G. Corcella et al., HERWIG 6: an event generator for hadron emission reactions with interfering gluons (including supersymmetric processes). JHEP 01, 010 (2001). https://doi.org/10.1088/ 1126-6708/2001/01/010. arXiv:hep-ph/0011363

11. M. Bahr, S. Gieseke, M.H. Seymour, Simulation of multiple partonic interactions in HERWIG++. JHEP 07, 076 (2008). https:// doi.org/10.1088/1126-6708/2008/07/076. arXiv:0803.3633

12. S. Gieseke, F. Loshaj, P. Kirchgaeßer, Soft and diffractive scattering with the cluster model in HERWIG. Eur. Phys. J. C 77, 156 (2017). https://doi.org/10.1140/epjc/s10052-017-4727-7. arXiv: 1612.04701

13. S. Gieseke, C. Rohr, A. Siodmok, Colour reconnections in HERWIG++. Eur. Phys. J. C 72, 2225 (2012). https://doi.org/10.1140/ epjc/s10052-012-2225-5. arXiv:1206.0041

14. ATLAS Collaboration, Rapidity gap cross sections measured with the ATLAS detector in pp collisions at $\sqrt{s}=7 \mathrm{TeV}$.
Eur. Phys. J. C 72, 1926 (2012). https://doi.org/10.1140/epjc/ s10052-012-1926-0. arXiv:1201.2808

15. CMS Collaboration, Measurement of diffraction dissociation cross sections in pp collisions at $\sqrt{s}=7 \mathrm{TeV}$. Phys. Rev. D 92, 012003 (2015). https://doi.org/10.1103/PhysRevD.92.012003. arXiv: 1503.08689

16. L.A. Harland-Lang, A.D. Martin, P. Motylinski, R.S. Thorne, Parton distributions in the LHC era: MMHT 2014 PDFs. Eur. Phys. J. C 75, 204 (2015). https://doi.org/10.1140/epjc/s10052-015-3397-6. arXiv: 1412.3989

17. ATLAS Collaboration, Charged-particle multiplicities in pp interactions measured with the ATLAS detector at the LHC. New J. Phys. 13, 053033 (2011). https://doi.org/10.1088/1367-2630/13/ 5/053033. arXiv: 1012.5104

18. NNPDF Collaboration, Parton distributions from high-precision collider data. Eur. Phys. J. C 77, 663 (2017). https://doi.org/10. 1140/epjc/s10052-017-5199-5. arXiv:1706.00428

19. CMS Collaboration, Extraction and validation of a new set of CMS PYTHIA 8 tunes from underlying-event measurements. Eur. Phys. J. C 80, 4 (2020). https://doi.org/10.1140/epjc/ s10052-019-7499-4. arXiv:1903.12179

20. CMS Collaboration, The CMS experiment at the CERN LHC. JINST 3, S08004 (2008). https://doi.org/10.1088/1748-0221/3/08/ S08004

21. B.R. Webber, A QCD model for jet fragmentation including soft gluon interference. Nucl. Phys. B 238, 492 (1984). https://doi.org/ 10.1016/0550-3213(84)90333-X

22. A. Buckley et al., Rivet user manual. Comput. Phys. Commun. 184, 2803 (2013). https://doi.org/10.1016/j.cpc.2013.05.021. arXiv: 1003.0694

23. CMS Collaboration, Measurement of the underlying event activity at the LHC at $7 \mathrm{TeV}$ and comparison with $0.9 \mathrm{TeV}$. CMS Physics Analysis Summary CMS-PAS-FSQ-12-020 (2012)

24. CMS Collaboration, Underlying event measurements with leading particles and jets in pp collisions at $\sqrt{s}=13 \mathrm{TeV}$. CMS Physics Analysis Summary CMS-PAS-FSQ-15-007 (2015)

25. CMS Collaboration, Measurement of the underlying event activity at the LHC with $\sqrt{s}=7 \mathrm{TeV}$ and comparison with $\sqrt{s}=0.9 \mathrm{TeV}$. JHEP 09, 109 (2011). https://doi.org/10.1007/JHEP09(2011)109. arXiv: 1107.0330

26. M. Cacciari, G.P. Salam, Dispelling the $N^{3}$ myth for the $k_{\mathrm{T}}$ jetfinder. Phys. Lett. B 641, 57 (2006). https://doi.org/10.1016/j. physletb.2006.08.037. arXiv:hep-ph/0512210

27. CMS Collaboration, Pseudorapidity distribution of charged hadrons in proton-proton collisions at $\sqrt{s}=13 \mathrm{TeV}$. Phys. Lett. B 751, 143 (2015). https://doi.org/10.1016/j.physletb.2015.10.004. arXiv: 1507.05915

28. CMS Collaboration, Transverse-momentum and pseudorapidity distributions of charged hadrons in pp collisions at $\sqrt{s}=7$ TeV. Phys. Rev. Lett. 105, 022002 (2010). https://doi.org/10.1103/ PhysRevLett.105.022002. arXiv:1005.3299

29. T. Sjöstrand, S. Mrenna, P.Z. Skands, PYTHIA 6.4 physics and manual. JHEP 05, 026 (2006). https://doi.org/10.1088/1126-6708/ 2006/05/026. arXiv:hep-ph/0603175

30. A. Buckley et al., Systematic event generator tuning for the LHC. Eur. Phys. J. C 65, 331 (2010). https://doi.org/10.1140/epjc/ s10052-009-1196-7. arXiv:0907.2973

31. CDF Collaboration, Study of the energy dependence of the underlying event in proton-antiproton collisions. Phys. Rev. D 92, 092009 (2015). https://doi.org/10.1103/PhysRevD.92.092009. arXiv: 1508.05340

32. ALEPH Collaboration, Studies of QCD at e+e- centre-of-mass energies between $91 \mathrm{GeV}$ and $209 \mathrm{GeV}$. Eur. Phys. J. C 35, 457 (2004). https://doi.org/10.1140/epjc/s2004-01891-4 
33. S. Catani, B.R. Webber, G. Marchesini, QCD coherent branching and semi-inclusive processes at large x. Nucl. Phys. B 349, 635 (1991). https://doi.org/10.1016/0550-3213(91)90390-J

34. D. Reichelt, P. Richardson, A. Siodmok, Improving the simulation of quark and gluon jets with HERWIG 7. Eur. Phys. J. C 77, 876 (2017). https://doi.org/10.1140/epjc/s10052-017-5374-8. arXiv: 1708.01491

35. S. Frixione, P. Nason, G. Ridolfi, A positive-weight next-toleading-order Monte Carlo for heavy flavour hadroproduction. JHEP 09, 126 (2007). https://doi.org/10.1088/1126-6708/2007/ 09/126. arXiv:0707.3088

36. CMS Collaboration, Investigations of the impact of the parton shower tuning in PYTHIA 8 in the modelling of $t \bar{t}$ at $\sqrt{s}=8$ and 13 TeV. CMS Physics Analysis Summary CMS-PAS-TOP-16-021 (2016)

37. B. Cooper et al., Importance of a consistent choice of $\alpha_{\mathrm{S}}$ in the matching of AlpGen and PYTHIA. Eur. Phys. J. C 72, 2078 (2012). https://doi.org/10.1140/epjc/s10052-012-2078-y. arXiv: 1109.5295

38. CMS Collaboration, Measurement of differential cross sections for the production of top quark pairs and of additional jets in lepton+jets events from pp collisions at $\sqrt{s}=13 \mathrm{TeV}$. Phys. Rev. D 97, 112003 (2018). https://doi.org/10.1103/PhysRevD.97.112003. arXiv: 1803.08856

39. M. Cacciari, G.P. Salam, G. Soyez, The anti- $k_{\mathrm{T}}$ jet clustering algorithm. JHEP 04, 063 (2008). https://doi.org/10.1088/1126-6708/ 2008/04/063. arXiv:0802.1189

40. M. Cacciari, G.P. Salam, G. Soyez, FastJet user manual. Eur. Phys. J. C 72, 1896 (2012). https://doi.org/10.1140/epjc/ s10052-012-1896-2. arXiv:1111.6097
41. CMS Collaboration, Measurements of differential cross sections of top quark pair production as a function of kinematic event variables in proton-proton collisions at $\sqrt{s}=13 \mathrm{TeV}$. JHEP 06, 002 (2018). https://doi.org/10.1007/JHEP06(2018)002. arXiv:1803.03991

42. CMS Collaboration, Measurement of jet substructure observables in $\mathrm{t} \overline{\mathrm{t}}$ events from proton-proton collisions at $\sqrt{s}=13 \mathrm{TeV}$. Phys. Rev. D 98, 092014 (2018). https://doi.org/10.1103/PhysRevD.98. 092014. arXiv: 1808.07340

43. CMS Collaboration, Shape, transverse size, and charged hadron multiplicity of jets in pp collisions at 7 TeV. JHEP 06, 160 (2012). https://doi.org/10.1007/JHEP06(2012)160. arXiv:1204.3170

44. R. Frederix, S. Frixione, Merging meets matching in MC@ NLO. JHEP 12, 061 (2012). https://doi.org/10.1007/JHEP12(2012)061. arXiv: 1209.6215

45. CMS Collaboration, Measurement of the underlying event activity in inclusive $\mathrm{Z}$ boson production in proton-proton collisions at $\sqrt{s}=13$ TeV. JHEP 07, 032 (2018). https://doi.org/10.1007/ JHEP07(2018)032. arXiv:1711.04299

46. CMS Collaboration, Measurement of differential cross sections for $\mathrm{Z}$ boson production in association with jets in proton-proton collisions at $\sqrt{s}=13$ TeV. Eur. Phys. J. C 78, 965 (2018). https://doi. org/10.1140/epjc/s10052-018-6373-0. arXiv:1804.05252

47. CMS Collaboration, Measurement of the differential cross sections for the associated production of a $W$ boson and jets in proton-proton collisions at $\sqrt{s}=13$ TeV. Phys. Rev. D 96, 072005 (2017). https:// doi.org/10.1103/PhysRevD.96.072005. arXiv:1707.05979

48. ATLAS Collaboration, Charged-particle distributions in $\sqrt{s}=13$ $\mathrm{TeV}$ pp interactions measured with the ATLAS detector at the LHC. Phys. Lett. B 758, 67 (2016). https://doi.org/10.1016/j.physletb. 2016.04.050. arXiv:1602.01633

\section{CMS Collaboration}

\section{Yerevan Physics Institute, Yerevan, Armenia}

A. M. Sirunyan ${ }^{\dagger}$, A. Tumasyan

\section{Institut für Hochenergiephysik, Vienna, Austria}

W. Adam (1), F. Ambrogi (1), T. Bergauer, M. Dragicevic (1), J. Erö, A. Escalante Del Valle (1), R. Frühwirth ${ }^{1}$, M. Jeitler ${ }^{1}$, N. Krammer, L. Lechner, D. Liko, T. Madlener, I. Mikulec, F. M. Pitters, N. Rad, J. Schieck (iD ${ }^{1}$, R. Schöfbeck (D), M. Spanring, S. Templ, W. Waltenberger (1), C.-E. Wulz ${ }^{1}$, M. Zarucki

\section{Institute for Nuclear Problems, Minsk, Belarus}

V. Chekhovsky, A. Litomin, V. Makarenko (1), J. Suarez Gonzalez

\section{Universiteit Antwerpen, Antwerpen, Belgium}

M. R. Darwish ${ }^{2}$, E. A. De Wolf, D. Di Croce, X. Janssen (1), T. Kello ${ }^{3}$, A. Lelek, M. Pieters, H. Rejeb Sfar, H. Van Haevermaet, P. Van Mechelen, S. Van Putte, N. Van Remortel (1)

\section{Vrije Universiteit Brussel, Brussels, Belgium}

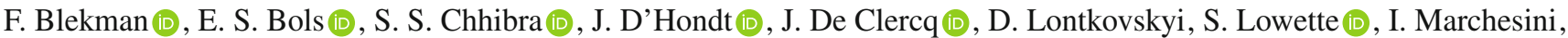
S. Moortgat (1), A. Morton (1D, Q. Python (1D, S. Tavernier, W. Van Doninck, P. Van Mulders

\section{Université Libre de Bruxelles, Brussels, Belgium}

D. Beghin, B. Bilin (D), B. Clerbaux (10, G. De Lentdecker, H. Delannoy, B. Dorney, L. Favart (1), A. Grebenyuk,

A. K. Kalsi (1), I. Makarenko (D), L. Moureaux, L. Pétré, A. Popov (1), N. Postiau, E. Starling (D), L. Thomas (1),

C. Vander Velde (1), P. Vanlaer (1D, D. Vannerom, L. Wezenbeek

\section{Ghent University, Ghent, Belgium}

T. Cornelis (1), D. Dobur, M. Gruchala, I. Khvastunov ${ }^{4}$, M. Niedziela, C. Roskas, K. Skovpen (1), M. Tytgat (1), W. Verbeke, B. Vermassen, M. Vit 
Université Catholique de Louvain, Louvain-la-Neuve, Belgium

G. Bruno, F. Bury, C. Caputo (10, P. David (1), C. Delaere (1), M. Delcourt, I. S. Donertas, A. Giammanco (1), V. Lemaitre, K. Mondal, J. Prisciandaro, A. Taliercio, M. Teklishyn, P. Vischia @ 1 , S. Wuyckens, J. Zobec

Centro Brasileiro de Pesquisas Fisicas, Rio de Janeiro, Brazil

G. A. Alves (1), G. Correia Silva, C. Hensel, A. Moraes (1)

Universidade do Estado do Rio de Janeiro, Rio de Janeiro, Brazil

W. L. Aldá Júnior (1), E. Belchior Batista Das Chagas, H. Brandao Malbouisson, W. Carvalho (1), J. Chinellato ${ }^{5}$, E. Coelho, E. M. Da Costa (D), G. G. Da Silveira (1) ${ }^{6}$, D. De Jesus Damiao (D), S. Fonseca De Souza (D), J. Martins ${ }^{7}$,

D. Matos Figueiredo, M. Medina Jaime ${ }^{8}$, M. Melo De Almeida, C. Mora Herrera (1), L. Mundim (1), H. Nogima,

P. Rebello Teles (10, L. J. Sanchez Rosas, A. Santoro, S. M. Silva Do Amaral (1), A. Sznajder (1), M. Thiel,

E. J. Tonelli Manganote ${ }^{5}$, F. Torres Da Silva De Araujo, A. Vilela Pereira (1)

Universidade Estadual Paulista ${ }^{a}$, Universidade Federal do ABC ${ }^{b}$, São Paulo, Brazil

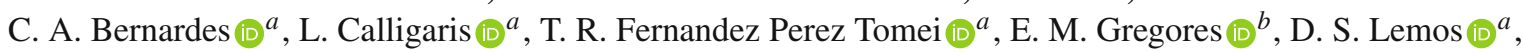

P. G. Mercadante (1) ${ }^{b}$, S. F. Novaes $\mathbb{1}^{a}$, S. S. Padula $\mathbb{1}^{a}$

Institute for Nuclear Research and Nuclear Energy, Bulgarian Academy of Sciences, Sofia, Bulgaria

A. Aleksandrov, G. Antchev, I. Atanasov, R. Hadjiiska, P. Iaydjiev, M. Misheva, M. Rodozov, M. Shopova, G. Sultanov

University of Sofia, Sofia, Bulgaria

M. Bonchev, A. Dimitrov, T. Ivanov, L. Litov (1), B. Pavlov, P. Petkov, A. Petrov

Beihang University, Beijing, China

W. Fang $\mathbb{B}^{3}$, Q. Guo, H. Wang, L. Yuan

Department of Physics, Tsinghua University, Beijing, China

M. Ahmad, Z. Hu (1), Y. Wang

Institute of High Energy Physics, Beijing, China

E. Chapon (1), G. M. Chen (1) ${ }^{9}$, H. S. Chen (1) ${ }^{9}$, M. Chen (1), D. Leggat, H. Liao, Z. Liu (1), R. Sharma (1), A. Spiezia,

J. Tao (1), J. Thomas-wilsker, J. Wang, H. Zhang, S. Zhang ${ }^{9}$, J. Zhao (1)

State Key Laboratory of Nuclear Physics and Technology, Peking University, Beijing, China

A. Agapitos, Y. Ban, C. Chen, A. Levin (1), Q. Li (1), M. Lu, X. Lyu, Y. Mao, S. J. Qian, D. Wang (1), Q. Wang (1), J. Xiao

Sun Yat-Sen University, Guangzhou, China

Z. You (1)

Institute of Modern Physics and Key Laboratory of Nuclear Physics and Ion-beam Application (MOE)-Fudan

University, Shanghai, China

X. $\mathrm{Gao}^{3}$

Zhejiang University, Hangzhou, China

M. Xiao (1)

Universidad de Los Andes, Bogotá, Colombia

C. Avila (1), A. Cabrera, C. Florez (10, J. Fraga, A. Sarkar, M. A. Segura Delgado

Universidad de Antioquia, Medellin, Colombia

J. Jaramillo, J. Mejia Guisao, F. Ramirez, J. D. Ruiz Alvarez (1), C. A. Salazar González, N. Vanegas Arbelaez

University of Split, Faculty of Electrical Engineering, Mechanical Engineering and Naval Architecture, Split, Croatia

D. Giljanovic, N. Godinovic (1), D. Lelas, I. Puljak (1), T. Sculac

University of Split, Faculty of Science, Split, Croatia

Z. Antunovic, M. Kovac

Institute Rudjer Boskovic, Zagreb, Croatia

V. Brigljevic (1), D. Ferencek (10, D. Majumder (10, B. Mesic, M. Roguljic, A. Starodumov ${ }^{10}$, T. Susa (1) 
University of Cyprus, Nicosia, Cyprus

M. W. Ather, A. Attikis, E. Erodotou, A. Ioannou, G. Kole @ (, M. Kolosova, S. Konstantinou, G. Mavromanolakis,

J. Mousa (10, C. Nicolaou, F. Ptochos (1), P. A. Razis, H. Rykaczewski, H. Saka (1D, D. Tsiakkouri

Charles University, Prague, Czech Republic

M. Finger ${ }^{11}$, M. Finger Jr. (10 ${ }^{11}$, A. Kveton, J. Tomsa

Escuela Politecnica Nacional, Quito, Ecuador

E. Ayala

Universidad San Francisco de Quito, Quito, Ecuador

E. Carrera Jarrin (1)

Academy of Scientific Research and Technology of the Arab Republic of Egypt, Egyptian Network of High Energy Physics, Cairo, Egypt

H. Abdalla (i) ${ }^{12}$, Y. Assran ${ }^{13,14}$, A. Mohamed (10) ${ }^{15}$

Center for High Energy Physics (CHEP-FU), Fayoum University, El-Fayoum, Egypt

M. A. Mahmoud (1), Y. Mohammed (1) ${ }^{16}$

National Institute of Chemical Physics and Biophysics, Tallinn, Estonia

S. Bhowmik (1), A. Carvalho Antunes De Oliveira (1), R. K. Dewanjee (1), K. Ehataht, M. Kadastik, M. Raidal (1), C. Veelken

Department of Physics, University of Helsinki, Helsinki, Finland

P. Eerola (1), L. Forthomme (1), H. Kirschenmann (1), K. Osterberg, M. Voutilainen (1)

Helsinki Institute of Physics, Helsinki, Finland

E. Brücken, F. Garcia, J. Havukainen, V. Karimäki, M. S. Kim, R. Kinnunen, T. Lampén, K. Lassila-Perini, S. Laurila,

S. Lehti, T. Lindén, H. Siikonen, E. Tuominen (1), J. Tuominiemi

Lappeenranta University of Technology, Lappeenranta, Finland

P. Luukka @, T. Tuuva

IRFU, CEA, Université Paris-Saclay, Gif-sur-Yvette, France

C. Amendola (1), M. Besancon, F. Couderc (1), M. Dejardin, D. Denegri, J. L. Faure, F. Ferri @ (, S. Ganjour, A. Givernaud,

P. Gras, G. Hamel de Monchenault (1), P. Jarry, B. Lenzi, E. Locci, J. Malcles, J. Rander, A. Rosowsky, M. Ö. Sahin (1),

A. Savoy-Navarro ${ }^{17}$, M. Titov (1), G. B. Yu (1)

Laboratoire Leprince-Ringuet, CNRS/IN2P3, Ecole Polytechnique, Institut Polytechnique de Paris, Palaiseau, France

S. Ahuja (1), F. Beaudette (1), M. Bonanomi, A. Buchot Perraguin, P. Busson, C. Charlot, O. Davignon, B. Diab,

G. Falmagne, R. Granier de Cassagnac (1), A. Hakimi, I. Kucher (1), A. Lobanov (1), C. Martin Perez, M. Nguyen (1),

C. Ochando, P. Paganini ([), J. Rembser, R. Salerno (1), J. B. Sauvan (1), Y. Sirois (1), A. Zabi, A. Zghiche (1)

Université de Strasbourg, CNRS, IPHC UMR 7178, Strasbourg, France

J.-L. Agram (1) ${ }^{18}$, J. Andrea, D. Bloch (1), G. Bourgatte, J.-M. Brom, E. C. Chabert, C. Collard (D) J.-C. Fontaine ${ }^{18}$, D. Gelé,

U. Goerlach, C. Grimault, A.-C. Le Bihan, P. Van Hove

Université de Lyon, Université Claude Bernard Lyon 1, CNRS-IN2P3, Institut de Physique Nucléaire de Lyon, Villeurbanne, France

E. Asilar (1), S. Beauceron (1), C. Bernet, G. Boudoul, C. Camen, A. Carle, N. Chanon (1), D. Contardo, P. Depasse (1),

H. El Mamouni, J. Fay, S. Gascon, M. Gouzevitch, B. Ille, Sa. Jain @i , I. B. Laktineh, H. Lattaud, A. Lesauvage,

M. Lethuillier (1), L. Mirabito, L. Torterotot, G. Touquet, M. Vander Donckt, S. Viret

Georgian Technical University, Tbilisi, Georgia

T. Toriashvili ${ }^{19}$, Z. Tsamalaidze $^{11}$

RWTH Aachen University, I. Physikalisches Institut, Aachen, Germany

L. Feld (1), K. Klein, M. Lipinski, D. Meuser, A. Pauls, M. Preuten, M. P. Rauch, J. Schulz, M. Teroerde (1) 


\section{RWTH Aachen University, III. Physikalisches Institut A, Aachen, Germany}

D. Eliseev, M. Erdmann (1), P. Fackeldey, B. Fischer, S. Ghosh (1), T. Hebbeker (1), K. Hoepfner, H. Keller,

L. Mastrolorenzo, M. Merschmeyer (1), A. Meyer, P. Millet, G. Mocellin, S. Mondal, S. Mukherjee (1), D. Noll, A. Novak,

T. Pook (1), A. Pozdnyakov (10, T. Quast, M. Radziej, Y. Rath, H. Reithler, J. Roemer, A. Schmidt (1), S. C. Schuler,

A. Sharma, S. Wiedenbeck, S. Zaleski

\section{RWTH Aachen University, III. Physikalisches Institut B, Aachen, Germany}

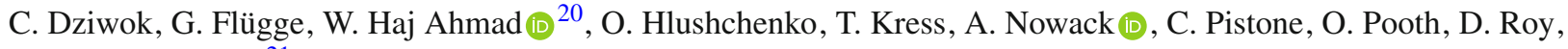
H. Sert, A. Stahl $\mathbb{D}^{21}$, T. Ziemons

\section{Deutsches Elektronen-Synchrotron, Hamburg, Germany}

H. Aarup Petersen, M. Aldaya Martin, P. Asmuss, I. Babounikau (®), S. Baxter, O. Behnke, A. Bermúdez Martínez, A. A. Bin Anuar (1), K. Borras ${ }^{22}$, V. Botta, D. Brunner, A. Campbell, A. Cardini, P. Connor, S. Consuegra Rodríguez (D), V. Danilov, A. De Wit (1), M. M. Defranchis, L. Didukh, D. Domínguez Damiani, G. Eckerlin, D. Eckstein, T. Eichhorn, A. Elwood (1), L. I. Estevez Banos, E. Gallo ${ }^{23}$, A. Geiser, A. Giraldi, A. Grohsjean (1), M. Guthoff, A. Harb (1), A. Jafari (1) ${ }^{24}$, N. Z. Jomhari (1), H. Jung, A. Kasem ${ }^{22}$, M. Kasemann (1), H. Kaveh, C. Kleinwort (), J. Knolle (1), D. Krücker, W. Lange, T. Lenz, J. Lidrych, K. Lipka, W. Lohmann ${ }^{25}$, R. Mankel, I.-A. Melzer-Pellmann, J. Metwally,

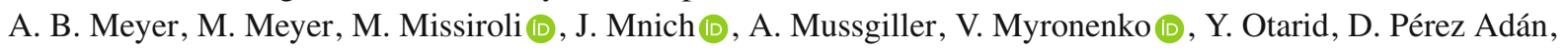
S. K. Pflitsch, D. Pitzl, A. Raspereza, A. Saggio, A. Saibel, M. Savitskyi, V. Scheurer, P. Schütze, C. Schwanenberger (1), R. Shevchenko (1), A. Singh, R. E. Sosa Ricardo (1), H. Tholen (1), N. Tonon (1), O. Turkot (1), A. Vagnerini, M. Van De Klundert, R. Walsh, D. Walter, Y. Wen (1), K. Wichmann, C. Wissing, S. Wuchterl, O. Zenaiev (1), R. Zlebcik (1)

\section{University of Hamburg, Hamburg, Germany}

R. Aggleton, S. Bein, L. Benato (10), A. Benecke, K. De Leo, T. Dreyer, A. Ebrahimi (1), M. Eich, F. Feindt, A. Fröhlich,

C. Garbers (1), E. Garutti (1), P. Gunnellini, J. Haller (10), A. Hinzmann (1), A. Karavdina, G. Kasieczka, R. Klanner (1),

R. Kogler, V. Kutzner, J. Lange (10, T. Lange, A. Malara, J. Multhaup, C. E. N. Niemeyer, A. Nigamova,

K. J. Pena Rodriguez, O. Rieger, P. Schleper, S. Schumann, J. Schwandt @ (1) D. Schwarz, J. Sonneveld, H. Stadie, G. Steinbrück, B. Vormwald (1), I. Zoi

\section{Karlsruher Institut fuer Technologie, Karlsruhe, Germany}

M. Baselga, S. Baur, J. Bechtel, T. Berger, E. Butz (1), R. Caspart, T. Chwalek, W. De Boer, A. Dierlamm, A. Droll, K. El Morabit, N. Faltermann (1), K. Flöh, M. Giffels, A. Gottmann, F. Hartmann (10) ${ }^{21}$, C. Heidecker, U. Husemann (1), M. A. Iqbal, I. Katkov ${ }^{26}$, P. Keicher, R. Koppenhöfer, S. Maier, M. Metzler, S. Mitra (1), M. U. Mozer, D. Müller, Th. Müller, M. Musich, G. Quast (10, K. Rabbertz (1), J. Rauser, D. Savoiu, D. Schäfer, M. Schnepf, M. Schröder (1), D. Seith, I. Shvetsov, H. J. Simonis, R. Ulrich @D, M. Wassmer, M. Weber, C. Wöhrmann, R. Wolf, S. Wozniewski

Institute of Nuclear and Particle Physics (INPP), NCSR Demokritos, Aghia Paraskevi, Greece

G. Anagnostou, P. Asenov, G. Daskalakis, T. Geralis, A. Kyriakis, D. Loukas, G. Paspalaki, A. Stakia

\section{National and Kapodistrian University of Athens, Athens, Greece}

M. Diamantopoulou, D. Karasavvas, G. Karathanasis, P. Kontaxakis, C. K. Koraka, A. Manousakis-katsikakis, A. Panagiotou, I. Papavergou, N. Saoulidou, K. Theofilatos, K. Vellidis, E. Vourliotis

National Technical University of Athens, Athens, Greece

G. Bakas, K. Kousouris (D), I. Papakrivopoulos, G. Tsipolitis, A. Zacharopoulou

University of Ioánnina, Ioánnina, Greece

I. Evangelou, C. Foudas, P. Gianneios, P. Katsoulis, P. Kokkas, S. Mallios, K. Manitara, N. Manthos, I. Papadopoulos, J. Strologas (1)

MTA-ELTE Lendület CMS Particle and Nuclear Physics Group, Eötvös Loránd University, Budapest, Hungary M. Bartók (1) ${ }^{27}$, R. Chudasama, M. Csanad (1), M. M. A. Gadallah ${ }^{28}$, S. Lökös ${ }^{29}$, P. Major, K. Mandal, A. Mehta (1), G. Pasztor (1), O. Surányi, G. I. Veres (1)

\section{Wigner Research Centre for Physics, Budapest, Hungary}

G. Bencze, C. Hajdu (1), D. Horvath ${ }^{30}$, F. Sikler (1), V. Veszpremi, G. Vesztergombi ${ }^{\dagger}$ 
Institute of Nuclear Research ATOMKI, Debrecen, Hungary

S. Czellar, J. Karancsi ${ }^{27}$, J. Molnar, Z. Szillasi, D. Teyssier

Institute of Physics, University of Debrecen, Debrecen, Hungary

P. Raics, Z. L. Trocsanyi (D), B. Ujvari

Eszterhazy Karoly University, Karoly Robert Campus, Gyongyos, Hungary

T. Csorgo, F. Nemes, T. Novak

Indian Institute of Science (IISc), Bangalore, India

S. Choudhury, J. R. Komaragiri (iD, D. Kumar, L. Panwar, P. C. Tiwari

National Institute of Science Education and Research, HBNI, Bhubaneswar, India

S. Bahinipati ${ }^{31}$, D. Dash (1), C. Kar, P. Mal, T. Mishra, V. K. Muraleedharan Nair Bindhu, A. Nayak ${ }^{32}$, D. K. Sahoo ${ }^{31}$,

N. Sur (1), S. K. Swain

Panjab University, Chandigarh, India

S. Bansal (1), S. B. Beri, V. Bhatnagar, S. Chauhan, N. Dhingra ${ }^{33}$, R. Gupta, A. Kaur, S. Kaur, P. Kumari, M. Lohan,

M. Meena, K. Sandeep, S. Sharma, J. B. Singh, A. K. Virdi

University of Delhi, Delhi, India

A. Ahmed, A. Bhardwaj, B. C. Choudhary (1), R. B. Garg, M. Gola, S. Keshri (1), A. Kumar, M. Naimuddin (1), P. Priyanka, K. Ranjan, A. Shah (1)

Saha Institute of Nuclear Physics, HBNI, Kolkata, India

M. Bharti ${ }^{34}$, R. Bhattacharya, S. Bhattacharya (1), D. Bhowmik, S. Dutta, S. Ghosh, B. Gomber ${ }^{35}$, M. Maity ${ }^{36}$, S. Nandan, P. Palit, A. Purohit, P. K. Rout, G. Saha, S. Sarkar, M. Sharan, B. Singh ${ }^{34}$, S. Thakur ${ }^{34}$

Indian Institute of Technology Madras, Madras, India

P. K. Behera (1), S. C. Behera, P. Kalbhor, A. Muhammad, R. Pradhan, P. R. Pujahari, A. Sharma, A. K. Sikdar

Bhabha Atomic Research Centre, Mumbai, India

D. Dutta, V. Jha, V. Kumar, D. K. Mishra, K. Naskar ${ }^{37}$, P. K. Netrakanti, L. M. Pant, P. Shukla (1)

Tata Institute of Fundamental Research-A, Mumbai, India

T. Aziz, M. A. Bhat, S. Dugad, R. Kumar Verma, U. Sarkar

Tata Institute of Fundamental Research-B, Mumbai, India

S. Banerjee, S. Bhattacharya, S. Chatterjee, P. Das, M. Guchait, S. Karmakar, S. Kumar, G. Majumder, K. Mazumdar, S. Mukherjee, D. Roy, N. Sahoo

Indian Institute of Science Education and Research (IISER), Pune, India

S. Dube (1D, B. Kansal, A. Kapoor (1), K. Kothekar (D), S. Pandey, A. Rane, A. Rastogi, S. Sharma (D)

Department of Physics, Isfahan University of Technology, Isfahan, Iran

H. Bakhshiansohi (iD ${ }^{38}$

Institute for Research in Fundamental Sciences (IPM), Tehran, Iran

S. Chenarani ${ }^{39}$, S. M. Etesami, M. Khakzad, M. Mohammadi Najafabadi (i)

University College Dublin, Dublin, Ireland

M. Felcini (iD, M. Grunewald (D)

INFN Sezione di Bari ${ }^{a}$, Università di Bari ${ }^{b}$, Politecnico di Bari $^{c}$, Bari, Italy

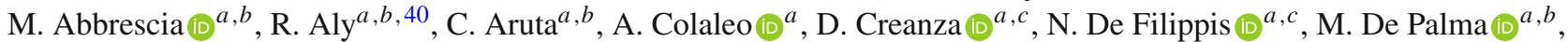

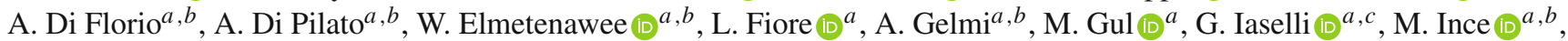

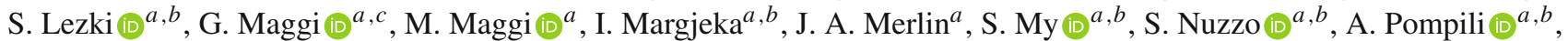

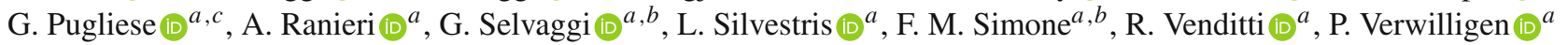

INFN Sezione di Bologna ${ }^{a}$, Università di Bologna ${ }^{b}$, Bologna, Italy

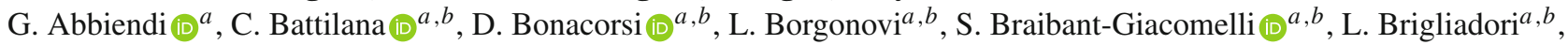




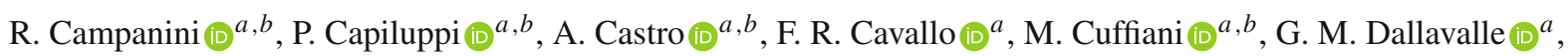

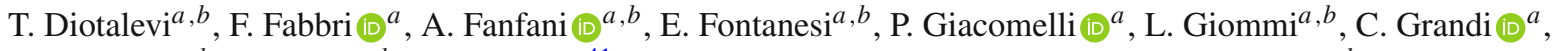

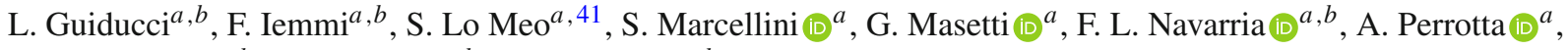

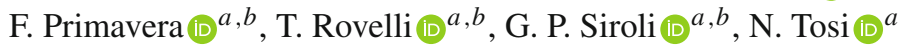

INFN Sezione di Catania ${ }^{a}$, Università di Catania ${ }^{b}$, Catania, Italy

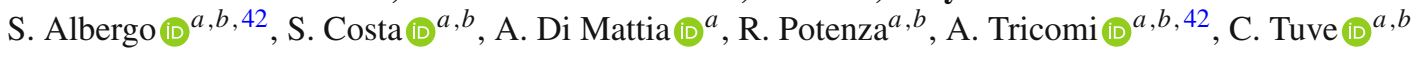

INFN Sezione di Firenze ${ }^{a}$, Università di Firenze ${ }^{b}$, Florence, Italy

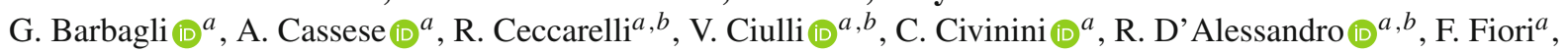

E. Focardi $\mathbb{C}^{a, b}$, G. Latino $\mathbb{C}^{a, b}$, P. Lenzi $\mathbb{C}^{a, b}$, M. Lizzo $^{a, b}$, M. Meschini $\mathbb{C}^{a}$, S. Paoletti $\mathbb{C}^{a}$, R. Seidita ${ }^{a, b}$,

G. Sguazzoni $\mathbb{(}^{a}{ }^{a}$, L. Viliani (i) ${ }^{a}$

INFN Laboratori Nazionali di Frascati, Frascati, Italy

L. Benussi (1), S. Bianco (1), D. Piccolo (1)

INFN Sezione di Genova ${ }^{a}$, Università di Genova ${ }^{b}$, Genoa, Italy

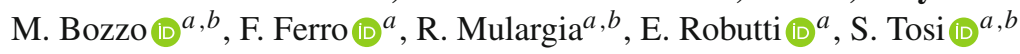

INFN Sezione di Milano-Bicocca ${ }^{a}$, Università di Milano-Bicocca ${ }^{b}$, Milan, Italy

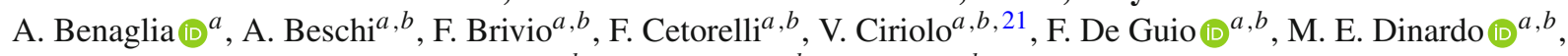

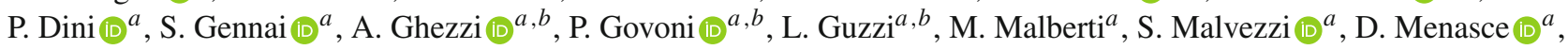

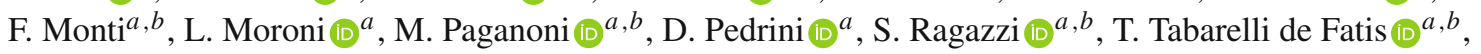

D. Valsecchi ${ }^{a, b, 21}$, D. Zuolo (iD ${ }^{a, b}$

INFN Sezione di Napoli ${ }^{a}$, Università di Napoli 'Federico II' ${ }^{b}$, Napoli, Italy, Università della Basilicata ${ }^{c}$, Potenza, Italy, Università G. Marconi ${ }^{d}$, Rome, Italy

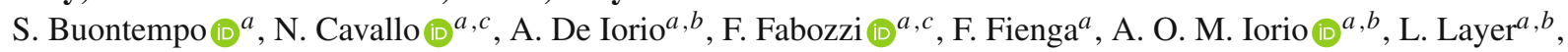

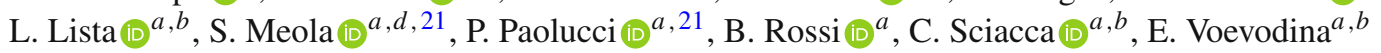

INFN Sezione di Padova ${ }^{a}$, Università di Padova ${ }^{b}$, Padova, Italy, Università di Trento ${ }^{c}$, Trento, Italy

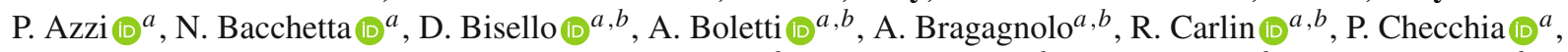

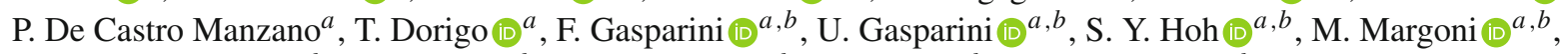

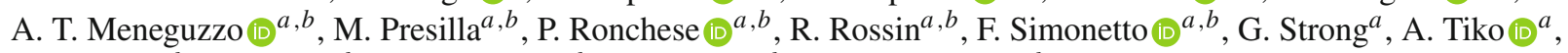

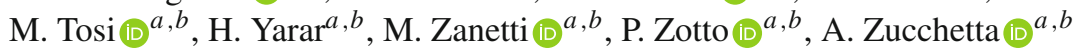

INFN Sezione di Pavia ${ }^{a}$, Università di Pavia ${ }^{b}$, Pavia, Italy

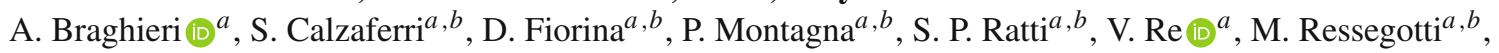

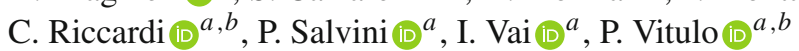

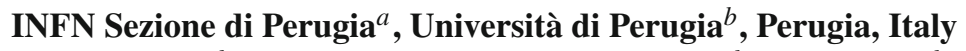

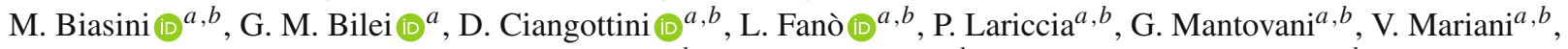

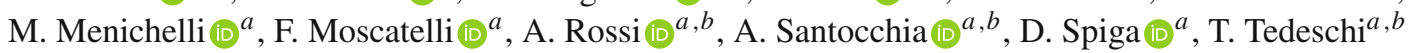

INFN Sezione di Pisa ${ }^{a}$, Università di Pisa ${ }^{b}$, Scuola Normale Superiore di Pisa ${ }^{c}$, Pisa, Italy

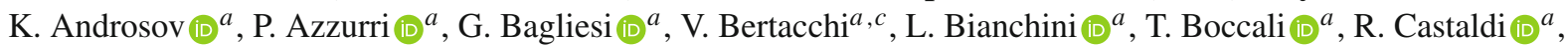

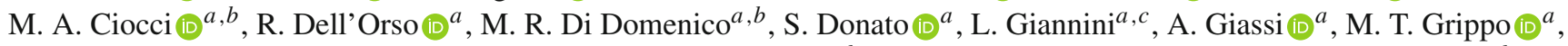
F. Ligabue $\mathbb{E}^{a, c}$, E. Manca $\mathbb{E}^{a, c}$, G. Mandorli ${ }^{a, c}$, A. Messineo $\mathbb{B}^{a, b}$, F. Palla $\mathbb{1}^{a}$, G. Ramirez-Sanchez ${ }^{a, c}$, A. Rizzi $\mathbb{D}^{a, b}$,

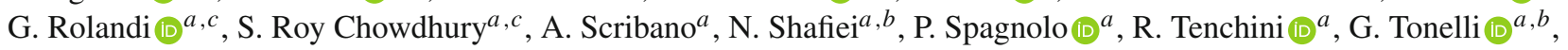

N. Turini ${ }^{a}$, A. Venturi ${ }^{a}{ }^{a}$, P. G. Verdini (i) ${ }^{a}$

INFN Sezione di Roma ${ }^{a}$, Sapienza Università di Roma ${ }^{b}$, Rome, Italy

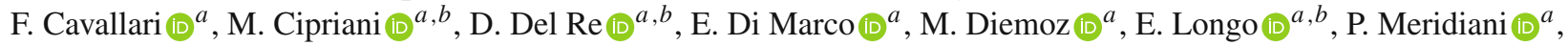

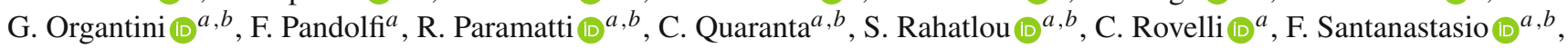
L. Soffi (10 ${ }^{a, b}, \mathrm{R}$. Tramontano ${ }^{a, b}$

INFN Sezione di Torino ${ }^{a}$, Università di Torino ${ }^{b}$, Torino, Italy, Università del Piemonte Orientale ${ }^{c}$, Novara, Italy

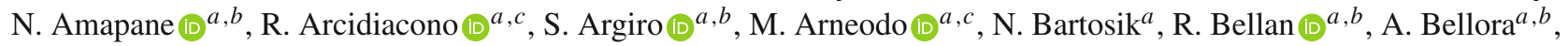

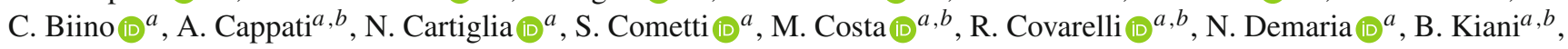




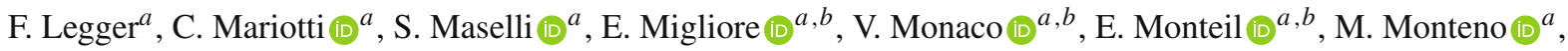

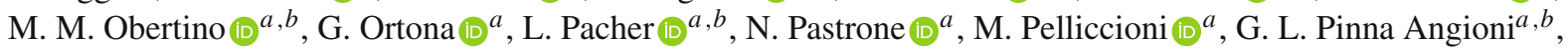

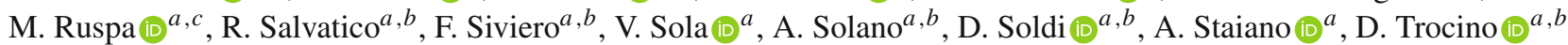

INFN Sezione di Trieste ${ }^{a}$, Università di Trieste ${ }^{b}$, Trieste, Italy

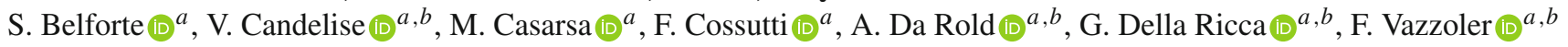

Kyungpook National University, Daegu, Korea

S. Dogra (1), C. Huh, B. Kim, D. H. Kim, G. N. Kim (1), J. Lee, S. W. Lee (1), C. S. Moon (1), Y. D. Oh (1), S. I. Pak,

B. C. Radburn-Smith, S. Sekmen (1), Y. C. Yang

Chonnam National University, Institute for Universe and Elementary Particles, Kwangju, Korea

H. Kim, D. H. Moon (1)

Hanyang University, Seoul, Korea

B. Francois, T. J. Kim (1), J. Park

Korea University, Seoul, Korea

S. Cho, S. Choi (1), Y. Go, S. Ha, B. Hong (1), K. Lee, K. S. Lee, J. Lim, J. Park, S. K. Park, J. Yoo

Kyung Hee University, Department of Physics, Seoul, Republic of Korea

J. Goh (D), A. Gurtu

Sejong University, Seoul, Korea

H. S. Kim (D), Y. Kim

Seoul National University, Seoul, Korea

J. Almond, J. H. Bhyun, J. Choi, S. Jeon, J. Kim, J. S. Kim, S. Ko, H. Kwon, H. Lee @i), K. Lee, S. Lee, K. Nam, B. H. Oh, M. Oh, S. B. Oh, H. Seo, U. K. Yang, I. Yoon (1)

University of Seoul, Seoul, Korea

D. Jeon, J. H. Kim, B. Ko, J. S. H. Lee (iD, I. C. Park, Y. Roh, D. Song, I. J. Watson (1)

Yonsei University, Department of Physics, Seoul, Korea

H. D. Yoo

Sungkyunkwan University, Suwon, Korea

Y. Choi, C. Hwang, Y. Jeong, H. Lee, Y. Lee, I. Yu

College of Engineering and Technology, American University of the Middle East (AUM), Kuwait

Y. Maghrbi

Riga Technical University, Riga, Latvia

V. Veckalns (1) ${ }^{43}$

Vilnius University, Vilnius, Lithuania

A. Juodagalvis (1), A. Rinkevicius (1), G. Tamulaitis

National Centre for Particle Physics, Universiti Malaya, Kuala Lumpur, Malaysia

W. A. T. Wan Abdullah, M. N. Yusli, Z. Zolkapli

Universidad de Sonora (UNISON), Hermosillo, Mexico

J. F. Benitez (1), A. Castaneda Hernandez (1), J. A. Murillo Quijada (1), L. Valencia Palomo (1)

Centro de Investigacion y de Estudios Avanzados del IPN, Mexico City, Mexico

H. Castilla-Valdez, E. De La Cruz-Burelo (1), I. Heredia-De La Cruz (10) ${ }^{44}$, R. Lopez-Fernandez, A. Sanchez-Hernandez (1)

Universidad Iberoamericana, Mexico City, Mexico

S. Carrillo Moreno, C. Oropeza Barrera, M. Ramirez-Garcia, F. Vazquez Valencia

Benemerita Universidad Autonoma de Puebla, Puebla, Mexico

J. Eysermans, I. Pedraza, H. A. Salazar Ibarguen, C. Uribe Estrada 
Universidad Autónoma de San Luis Potosí, San Luis Potosí, Mexico

A. Morelos Pineda (1)

University of Montenegro, Podgorica, Montenegro

J. Mijuskovic ${ }^{4}$, N. Raicevic

University of Auckland, Auckland, New Zealand

D. Krofcheck (D)

University of Canterbury, Christchurch, New Zealand

S. Bheesette, P. H. Butler (1)

National Centre for Physics, Quaid-I-Azam University, Islamabad, Pakistan

A. Ahmad, M. I. Asghar, M. I. M. Awan, Q. Hassan, H. R. Hoorani, W. A. Khan, M. A. Shah, M. Shoaib (1), M. Waqas

AGH University of Science and Technology Faculty of Computer Science, Electronics and Telecommunications, Krakow, Poland

V. Avati, L. Grzanka, M. Malawski

National Centre for Nuclear Research, Swierk, Poland

H. Bialkowska, M. Bluj (1), B. Boimska, T. Frueboes, M. Górski, M. Kazana, M. Szleper, P. Traczyk, P. Zalewski

Institute of Experimental Physics, Faculty of Physics, University of Warsaw, Warsaw, Poland

K. Bunkowski, A. Byszuk ${ }^{45}$, K. Doroba, A. Kalinowski (i), M. Konecki (1), J. Krolikowski, M. Olszewski, M. Walczak

Laboratório de Instrumentação e Física Experimental de Partículas, Lisbon, Portugal

M. Araujo, P. Bargassa (1), D. Bastos, P. Faccioli (1), M. Gallinaro (), J. Hollar, N. Leonardo (1), T. Niknejad, J. Seixas (1),

K. Shchelina, O. Toldaiev (D), J. Varela (1)

Joint Institute for Nuclear Research, Dubna, Russia

S. Afanasiev, V. Alexakhin (1), P. Bunin, M. Gavrilenko, I. Golutvin, I. Gorbunov, V. Karjavine, A. Lanev, A. Malakhov, V. Matveev ${ }^{46,47}$, V. V. Mitsyn, P. Moisenz, V. Palichik, V. Perelygin, M. Savina, S. Shmatov, S. Shulha, V. Smirnov,

O. Teryaev, V. Trofimov, N. Voytishin, B. S. Yuldashev ${ }^{48}$, A. Zarubin

Petersburg Nuclear Physics Institute, Gatchina (St. Petersburg), Russia

G. Gavrilov, V. Golovtcov, Y. Ivanov, V. Kim ${ }^{49}$, E. Kuznetsova ${ }^{50}$, V. Murzin, V. Oreshkin, I. Smirnov, D. Sosnov,

V. Sulimov, L. Uvarov, S. Volkov, A. Vorobyev

Institute for Nuclear Research, Moscow, Russia

Yu. Andreev (1), A. Dermenev, S. Gninenko (1), N. Golubev, A. Karneyeu, M. Kirsanov, N. Krasnikov, A. Pashenkov,

G. Pivovarov (iD, D. Tlisov ${ }^{\dagger}$, A. Toropin

Institute for Theoretical and Experimental Physics named by A.I. Alikhanov of NRC 'Kurchatov Institute', Moscow, Russia

V. Epshteyn, V. Gavrilov, N. Lychkovskaya, A. Nikitenko ${ }^{51}$, V. Popov, I. Pozdnyakov, G. Safronov, A. Spiridonov,

A. Stepennov, M. Toms, E. Vlasov (1), A. Zhokin

Moscow Institute of Physics and Technology, Moscow, Russia

T. Aushev

National Research Nuclear University 'Moscow Engineering Physics Institute' (MEPhI), Moscow, Russia

M. Chadeeva (i) ${ }^{52}$, A. Oskin, P. Parygin, S. Polikarpov (1) ${ }^{52}$, E. Zhemchugov (D)

P.N. Lebedev Physical Institute, Moscow, Russia

V. Andreev, M. Azarkin, I. Dremin, M. Kirakosyan, A. Terkulov

Skobeltsyn Institute of Nuclear Physics, Lomonosov Moscow State University, Moscow, Russia

A. Belyaev, E. Boos (1), V. Bunichev, M. Dubinin (1) ${ }^{53}$, L. Dudko (1), V. Klyukhin (1), O. Kodolova, I. Lokhtin (1),

S. Obraztsov, M. Perfilov, S. Petrushanko, V. Savrin, A. Snigirev (1) 
Novosibirsk State University (NSU), Novosibirsk, Russia

V. Blinov ${ }^{54}$, T. Dimova ${ }^{54}$, L. Kardapoltsev ${ }^{54}$, I. Ovtin ${ }^{54}$, Y. Skovpen (D) $^{54}$

Institute for High Energy Physics of National Research Centre 'Kurchatov Institute', Protvino, Russia I. Azhgirey (1D, I. Bayshev, V. Kachanov, A. Kalinin, D. Konstantinov, V. Petrov, R. Ryutin, A. Sobol, S. Troshin (1), N. Tyurin, A. Uzunian, A. Volkov

National Research Tomsk Polytechnic University, Tomsk, Russia

A. Babaev, A. Iuzhakov, V. Okhotnikov, L. Sukhikh

Tomsk State University, Tomsk, Russia

V. Borchsh, V. Ivanchenko (1), E. Tcherniaev

University of Belgrade: Faculty of Physics and VINCA Institute of Nuclear Sciences, Belgrade, Serbia P. Adzic ${ }^{55}$, P. Cirkovic (1), M. Dordevic (1), P. Milenovic, J. Milosevic (1)

Centro de Investigaciones Energéticas Medioambientales y Tecnológicas (CIEMAT), Madrid, Spain M. Aguilar-Benitez, J. Alcaraz Maestre (1), A. Álvarez Fernández, I. Bachiller, M. Barrio Luna, C. F. Bedoya (1), J. A. Brochero Cifuentes (10, C. A. Carrillo Montoya, M. Cepeda (1), M. Cerrada, N. Colino (1), B. De La Cruz, A. Delgado Peris (10), J. P. Fernández Ramos (1), J. Flix (1), M. C. Fouz, A. García Alonso, O. Gonzalez Lopez (1), S. Goy Lopez, J. M. Hernandez (10, M. I. Josa, J. León Holgado, D. Moran, Á. Navarro Tobar,

A. Pérez-Calero Yzquierdo (1), J. Puerta Pelayo (1), I. Redondo (1), L. Romero, S. Sánchez Navas, M. S. Soares (1),

A. Triossi (1), L. Urda Gómez, C. Willmott

Universidad Autónoma de Madrid, Madrid, Spain

C. Albajar, J. F. de Trocóniz, R. Reyes-Almanza

Universidad de Oviedo, Instituto Universitario de Ciencias y Tecnologías Espaciales de Asturias (ICTEA), Oviedo, Spain

B. Alvarez Gonzalez, J. Cuevas (1), C. Erice, J. Fernandez Menendez (1), S. Folgueras (1), I. Gonzalez Caballero (1),

E. Palencia Cortezon (1), C. Ramón Álvarez, J. Ripoll Sau, V. Rodríguez Bouza (1), S. Sanchez Cruz (10, A. Trapote

Instituto de Física de Cantabria (IFCA), CSIC-Universidad de Cantabria, Santander, Spain

I. J. Cabrillo, A. Calderon (1), B. Chazin Quero, J. Duarte Campderros (1), M. Fernandez (1), P. J. Fernández Manteca (1),

G. Gomez, C. Martinez Rivero, P. Martinez Ruiz del Arbol (1), F. Matorras (1), J. Piedra Gomez (10, C. Prieels,

F. Ricci-Tam (1), T. Rodrigo (1), A. Ruiz-Jimeno (1), L. Russo (i) ${ }^{56}$, L. Scodellaro (1), I. Vila, J. M. Vizan Garcia (1)

University of Colombo, Colombo, Sri Lanka

MK Jayananda, B. Kailasapathy ${ }^{57}$, D. U. J. Sonnadara, D. D. C. Wickramarathna

University of Ruhuna, Department of Physics, Matara, Sri Lanka

W. G. D. Dharmaratna (10, K. Liyanage, N. Perera, N. Wickramage

CERN, European Organization for Nuclear Research, Geneva, Switzerland

T. K. Aarrestad, D. Abbaneo, B. Akgun, E. Auffray, G. Auzinger, J. Baechler, P. Baillon, A. H. Ball, D. Barney,

J. Bendavid, N. Beni, M. Bianco (1), A. Bocci, P. Bortignon (1), E. Bossini, E. Brondolin, T. Camporesi, G. Cerminara,

L. Cristella (1), D. d'Enterria (1), A. Dabrowski, N. Daci, V. Daponte, A. David (1), A. De Roeck (10), M. Deile,

R. Di Maria (1), M. Dobson, M. Dünser (1), N. Dupont, A. Elliott-Peisert, N. Emriskova, F. Fallavollita ${ }^{58}$, D. Fasanella (1),

S. Fiorendi (1), G. Franzoni (1), J. Fulcher (1), W. Funk, S. Giani, D. Gigi, K. Gill, F. Glege, L. Gouskos, M. Guilbaud,

D. Gulhan, M. Haranko (1), J. Hegeman (1), Y. Iiyama (1), V. Innocente, T. James, P. Janot (1), J. Kaspar, J. Kieseler (1),

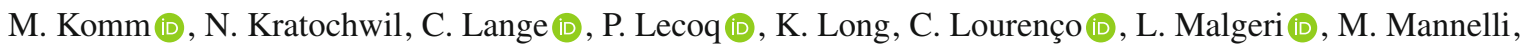

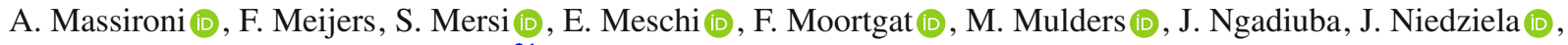

S. Orfanelli, L. Orsini, F. Pantaleo (1) ${ }^{21}$, L. Pape, E. Perez, M. Peruzzi, A. Petrilli, G. Petrucciani (i), A. Pfeiffer (1),

M. Pierini (1), D. Rabady (1), A. Racz, M. Rieger (1), M. Rovere, H. Sakulin, J. Salfeld-Nebgen (1), S. Scarfi, C. Schäfer,

C. Schwick, M. Selvaggi, A. Sharma, P. Silva (1), W. Snoeys (1), P. Sphicas (1) ${ }^{59}$, J. Steggemann (1), S. Summers,

V. R. Tavolaro (1), D. Treille, A. Tsirou, G. P. Van Onsem (1), A. Vartak (1), M. Verzetti, K. A. Wozniak, W. D. Zeuner

Paul Scherrer Institut, Villigen, Switzerland

L. Caminada $~^{60}$, W. Erdmann, R. Horisberger, Q. Ingram, H. C. Kaestli, D. Kotlinski, U. Langenegger, T. Rohe 
ETH Zurich-Institute for Particle Physics and Astrophysics (IPA), Zurich, Switzerland

M. Backhaus (1), P. Berger, A. Calandri, N. Chernyavskaya, G. Dissertori (1), M. Dittmar, M. Donegà, C. Dorfer, T. Gadek, T. A. Gómez Espinosa (1) , C. Grab (1), D. Hits, W. Lustermann, A.-M. Lyon, R. A. Manzoni (1), M. T. Meinhard, F. Micheli, F. Nessi-Tedaldi, F. Pauss, V. Perovic, G. Perrin, L. Perrozzi, S. Pigazzini (1), M. G. Ratti (1), M. Reichmann, C. Reissel, T. Reitenspiess, B. Ristic, D. Ruini, D. A. Sanz Becerra, M. Schönenberger (1), L. Shchutska (1), V. Stampf, M. L. Vesterbacka Olsson, R. Wallny (1), D. H. Zhu

Universität Zürich, Zurich, Switzerland

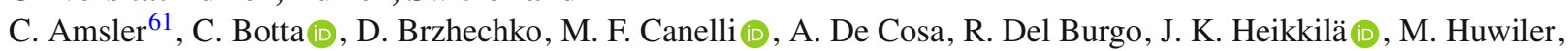
A. Jofrehei, B. Kilminster (1), S. Leontsinis (1), A. Macchiolo, P. Meiring, V. M. Mikuni, U. Molinatti, I. Neutelings, G. Rauco, A. Reimers, P. Robmann, K. Schweiger (1), Y. Takahashi (1), S. Wertz (1)

National Central University, Chung-Li, Taiwan

C. Adloff ${ }^{62}$, C. M. Kuo, W. Lin, A. Roy, T. Sarkar $\mathbb{1}^{36}$, S. S. Yu

National Taiwan University (NTU), Taipei, Taiwan

L. Ceard, P. Chang (1), Y. Chao, K. F. Chen, P. H. Chen, W.-S. Hou (), Y. Y. Li, R.-S. Lu, E. Paganis, A. Psallidas, A. Steen, E. Yazgan (1)

Chulalongkorn University, Faculty of Science, Department of Physics, Bangkok, Thailand

B. Asavapibhop (10, C. Asawatangtrakuldee, N. Srimanobhas

Çukurova University, Physics Department, Science and Art Faculty, Adana, Turkey

F. Boran, S. Damarseckin ${ }^{63}$, Z. S. Demiroglu (1), F. Dolek, C. Dozen ${ }^{64}$, I. Dumanoglu ${ }^{65}$, E. Eskut, G. Gokbulut, Y. Guler,

E. Gurpinar Guler ${ }^{66}$, I. Hos $^{67}$, C. Isik, E. E. Kangal ${ }^{68}$, O. Kara, A. Kayis Topaksu, U. Kiminsu @ ( ) G. Onengut,

K. Ozdemir ${ }^{69}$, A. Polatoz, A. E. Simsek, B. Tali ${ }^{70}$, U. G. Tok, S. Turkcapar, I. S. Zorbakir (1), C. Zorbilmez

Middle East Technical University, Physics Department, Ankara, Turkey

B. Isildak ${ }^{71}$, G. Karapinar ${ }^{72}$, K. Ocalan (1) ${ }^{73}$, M. Yalvac ${ }^{74}$

Bogazici University, Istanbul, Turkey

I. O. Atakisi, E. Gülmez (1), M. Kaya ${ }^{75}$, O. Kaya ${ }^{76}$, Ö. Özçelik, S. Tekten ${ }^{77}$, E. A. Yetkin (1) ${ }^{78}$

Istanbul Technical University, Istanbul, Turkey

A. Cakir (1), K. Cankocak ${ }^{65}$, Y. Komurcu, S. Sen (10) ${ }^{79}$

Istanbul University, Istanbul, Turkey

F. Aydogmus Sen, S. Cerci ${ }^{70}$, B. Kaynak, S. Ozkorucuklu, D. Sunar Cerci ${ }^{70}$

Institute for Scintillation Materials of National Academy of Science of Ukraine, Kharkov, Ukraine

B. Grynyov

National Scientific Center, Kharkov Institute of Physics and Technology, Kharkov, Ukraine

L. Levchuk (1)

University of Bristol, Bristol, UK

E. Bhal, S. Bologna, J. J. Brooke (1), E. Clement (1), D. Cussans, H. Flacher (1), J. Goldstein (1), G. P. Heath, H. F. Heath (1),

L. Kreczko (1), B. Krikler (1), S. Paramesvaran, T. Sakuma @i], S. Seif El Nasr-Storey, V. J. Smith, J. Taylor, A. Titterton

Rutherford Appleton Laboratory, Didcot, UK

K. W. Bell, A. Belyaev (1) ${ }^{80}$, C. Brew (1), R. M. Brown, D. J. A. Cockerill, K. V. Ellis, K. Harder, S. Harper, J. Linacre (1), K. Manolopoulos, D. M. Newbold (1), E. Olaiya, D. Petyt, T. Reis (1), T. Schuh, C. H. Shepherd-Themistocleous,

A. Thea $\mathbb{O}^{-}$, I. R. Tomalin, T. Williams

\section{Imperial College, London, UK}

R. Bainbridge (1), P. Bloch, S. Bonomally, J. Borg (1), S. Breeze, O. Buchmuller, A. Bundock (1), V. Cepaitis, G. S. Chahal ${ }^{81}$, D. Colling, P. Dauncey (1), G. Davies, M. Della Negra (1), P. Everaerts (1), G. Fedi (1), G. Hall (1), G. Iles, J. Langford, L. Lyons, A.-M. Magnan, S. Malik, A. Martelli (i), V. Milosevic (1), J. Nash (1) ${ }^{82}$, V. Palladino (1), M. Pesaresi, D. M. Raymond, A. Richards, A. Rose, E. Scott (10, C. Seez, A. Shtipliyski, M. Stoye, A. Tapper (10, K. Uchida,

T. Virdee (1) ${ }^{21}$, N. Wardle (1) , S. N. Webb, D. Winterbottom, A. G. Zecchinelli, S. C. Zenz (1) 


\section{Brunel University, Uxbridge, UK}

J. E. Cole (1), P. R. Hobson (1), A. Khan, P. Kyberd (1), C. K. Mackay, I. D. Reid ([D, L. Teodorescu, S. Zahid

Baylor University, Waco, USA

A. Brinkerhoff (1), K. Call, B. Caraway, J. Dittmann, K. Hatakeyama, A. R. Kanuganti, C. Madrid, B. McMaster,

N. Pastika, S. Sawant, C. Smith

Catholic University of America, Washington, DC, USA

R. Bartek (10, A. Dominguez (10, R. Uniyal, A. M. Vargas Hernandez

The University of Alabama, Tuscaloosa, USA

A. Buccilli (1), O. Charaf, S. I. Cooper, S. V. Gleyzer, C. Henderson (D), P. Rumerio, C. West

Boston University, Boston, USA

A. Akpinar, A. Albert @ D, D. Arcaro, C. Cosby, Z. Demiragli, D. Gastler, C. Richardson, J. Rohlf, K. Salyer, D. Sperka, D. Spitzbart (1D, I. Suarez, S. Yuan, D. Zou

\section{Brown University, Providence, USA}

G. Benelli, B. Burkle (1), X. Coubez ${ }^{22}$, D. Cutts (1), Y. t. Duh, M. Hadley, U. Heintz, J. M. Hogan (1) ${ }^{83}$, K. H. M. Kwok,

E. Laird, G. Landsberg (1), K. T. Lau, J. Lee, M. Narain, S. Sagir (1) ${ }^{84}$, R. Syarif (1), E. Usai (1), W. Y. Wong, D. Yu, W. Zhang

University of California, Davis, Davis, USA

R. Band, C. Brainerd (1), R. Breedon, M. Calderon De La Barca Sanchez, M. Chertok, J. Conway (1), R. Conway, P. T. Cox,

R. Erbacher, C. Flores, G. Funk, F. Jensen, W. Ko ${ }^{\dagger}$, O. Kukral, R. Lander, M. Mulhearn, D. Pellett, J. Pilot, M. Shi,

D. Taylor (1), K. Tos, M. Tripathi (1), Y. Yao, F. Zhang (1)

University of California, Los Angeles, USA

M. Bachtis, R. Cousins (1), A. Dasgupta, A. Florent (10, D. Hamilton, J. Hauser (1), M. Ignatenko, T. Lam, N. Mccoll,

W. A. Nash, S. Regnard (1), D. Saltzberg (1), C. Schnaible, B. Stone, V. Valuev

University of California, Riverside, Riverside, USA

K. Burt, Y. Chen, R. Clare (ㄹ, J. W. Gary (1), S. M. A. Ghiasi Shirazi, G. Hanson, G. Karapostoli, O. R. Long (D),

N. Manganelli, M. Olmedo Negrete, M. I. Paneva, W. Si, S. Wimpenny, Y. Zhang

University of California, San Diego, La Jolla, USA

J. G. Branson, P. Chang, S. Cittolin, S. Cooperstein, N. Deelen, M. Derdzinski, J. Duarte (1), R. Gerosa (1), D. Gilbert (1),

B. Hashemi, D. Klein (1), V. Krutelyov (1), J. Letts (1), M. Masciovecchio, S. May, S. Padhi, M. Pieri (1), V. Sharma (1),

M. Tadel, F. Würthwein (1), A. Yagil(1)

Department of Physics, University of California, Santa Barbara, Santa Barbara, USA

N. Amin, C. Campagnari, M. Citron, A. Dorsett, V. Dutta, J. Incandela (D), B. Marsh, H. Mei, A. Ovcharova, H. Qu (1),

M. Quinnan, J. Richman, U. Sarica (D), D. Stuart, S. Wang

\section{California Institute of Technology, Pasadena, USA}

D. Anderson, A. Bornheim (1), O. Cerri, I. Dutta, J. M. Lawhorn (1), N. Lu (1), J. Mao, H. B. Newman (1), T. Q. Nguyen (1),

J. Pata, M. Spiropulu (1), J. R. Vlimant (1), S. Xie (1) , Z. Zhang (1), R. Y. Zhu (1)

\section{Carnegie Mellon University, Pittsburgh, USA}

J. Alison, M. B. Andrews, T. Ferguson (1), T. Mudholkar, M. Paulini (i), M. Sun, I. Vorobiev

University of Colorado Boulder, Boulder, USA

J. P. Cumalat, W. T. Ford (1D, E. MacDonald, T. Mulholland, R. Patel, A. Perloff (1), K. Stenson (1), K. A. Ulmer (1),

S. R. Wagner (1)

\section{Cornell University, Ithaca, USA}

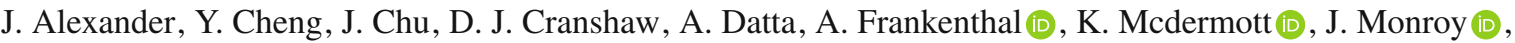

J. R. Patterson (1), D. Quach (1), A. Ryd, W. Sun (1), S. M. Tan, Z. Tao, J. Thom, P. Wittich (1), M. Zientek

Fermi National Accelerator Laboratory, Batavia, USA

S. Abdullin (1), M. Albrow (1), M. Alyari, G. Apollinari, A. Apresyan (1), A. Apyan (1), S. Banerjee, L. A. T. Bauerdick (1), 
A. Beretvas (1), D. Berry (1), J. Berryhill (1), P. C. Bhat, K. Burkett (1), J. N. Butler, A. Canepa, G. B. Cerati (1),

H. W. K. Cheung (1), F. Chlebana, M. Cremonesi, V. D. Elvira (1), J. Freeman, Z. Gecse, E. Gottschalk (1), L. Gray, D. Green, S. Grünendahl (1), O. Gutsche (1), R. M. Harris (1), S. Hasegawa, R. Heller, T. C. Herwig, J. Hirschauer (1), B. Jayatilaka (1),

S. Jindariani, M. Johnson, U. Joshi, P. Klabbers (1), T. Klijnsma, B. Klima (1), M. J. Kortelainen (ㅇ, S. Lammel(1),

D. Lincoln (1), R. Lipton, M. Liu, T. Liu, J. Lykken, K. Maeshima, D. Mason, P. McBride (1), P. Merkel, S. Mrenna (1),

S. Nahn, V. O’Dell, V. Papadimitriou, K. Pedro (1), C. Pena (1) ${ }^{53}$, O. Prokofyev, F. Ravera (1), A. Reinsvold Hall (1),

L. Ristori (1), B. Schneider (1), E. Sexton-Kennedy (1), N. Smith, A. Soha (1), W. J. Spalding (), L. Spiegel, S. Stoynev (1),

J. Strait (1), L. Taylor (D), S. Tkaczyk, N. V. Tran, L. Uplegger (10, E. W. Vaandering (1), H. A. Weber (1), A. Woodard

University of Florida, Gainesville, USA

D. Acosta, P. Avery, D. Bourilkov (1), L. Cadamuro (1), V. Cherepanov, F. Errico, R. D. Field, D. Guerrero, B. M. Joshi, M. Kim, J. Konigsberg, A. Korytov, K. H. Lo, K. Matchev, N. Menendez, G. Mitselmakher (1), D. Rosenzweig, K. Shi (1), J. Wang (1), S. Wang (1), X. Zuo

\section{Florida State University, Tallahassee, USA}

T. Adams (1), A. Askew, D. Diaz, R. Habibullah (1), S. Hagopian (1), V. Hagopian, K. F. Johnson, R. Khurana, T. Kolberg (1), G. Martinez, H. Prosper, C. Schiber, R. Yohay (1), J. Zhang

Florida Institute of Technology, Melbourne, USA

M. M. Baarmand (1), S. Butalla, T. Elkafrawy (1) ${ }^{85}$, M. Hohlmann (1), D. Noonan, M. Rahmani, M. Saunders, F. Yumiceva (D) University of Illinois at Chicago (UIC), Chicago, USA

M. R. Adams, L. Apanasevich (1), H. Becerril Gonzalez, R. Cavanaugh (1), X. Chen (1), S. Dittmer, O. Evdokimov (1),

C. E. Gerber (1), D. A. Hangal, D. J. Hofman (1), C. Mills (D), G. Oh, T. Roy, M. B. Tonjes, N. Varelas, J. Viinikainen (1),

X. Wang, Z. Wu (1)

The University of Iowa, Iowa City, USA

M. Alhusseini, K. Dilsiz ${ }^{86}$, S. Durgut, R. P. Gandrajula (1), M. Haytmyradov, V. Khristenko, O. K. Köseyan, J.-P. Merlo, A. Mestvirishvili ${ }^{87}$, A. Moeller, J. Nachtman, H. Ogul (10 ${ }^{88}$, Y. Onel, F. Ozok ${ }^{89}$, A. Penzo, C. Snyder, E. Tiras, J. Wetzel (1), K. Yi ${ }^{90}$

\section{Johns Hopkins University, Baltimore, USA}

O. Amram, B. Blumenfeld (1), L. Corcodilos, M. Eminizer, A. V. Gritsan (1), S. Kyriacou, P. Maksimovic, C. Mantilla (1),

J. Roskes (1), M. Swartz, T. Á. Vámi (1)

The University of Kansas, Lawrence, USA

C. Baldenegro Barrera, P. Baringer (1), A. Bean (1), A. Bylinkin (1), T. Isidori, S. Khalil (1), J. King, G. Krintiras (1),

A. Kropivnitskaya, C. Lindsey, N. Minafra (1), M. Murray, C. Rogan (1), C. Royon, S. Sanders, E. Schmitz,

J. D. Tapia Takaki (1), Q. Wang (1), J. Williams, G. Wilson (1)

\section{Kansas State University, Manhattan, USA}

S. Duric, A. Ivanov (1), K. Kaadze, D. Kim, Y. Maravin (1D, T. Mitchell, A. Modak, A. Mohammadi

\section{Lawrence Livermore National Laboratory, Livermore, USA}

F. Rebassoo, D. Wright

\section{University of Maryland, College Park, USA}

E. Adams, A. Baden, O. Baron, A. Belloni (ib, S. C. Eno @i), Y. Feng, N. J. Hadley, S. Jabeen, G. Y. Jeng (1), R. G. Kellogg, T. Koeth, A. C. Mignerey, S. Nabili, M. Seidel $@$, A. Skuja, S. C. Tonwar, L. Wang, K. Wong

\section{Massachusetts Institute of Technology, Cambridge, USA}

D. Abercrombie, B. Allen (), R. Bi, S. Brandt, W. Busza (i), I. A. Cali, Y. Chen (1), M. D’Alfonso (iD, G. Gomez Ceballos,

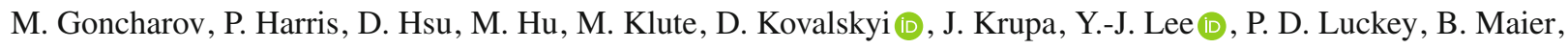

A. C. Marini (1), C. Mcginn, C. Mironov, S. Narayanan (1), X. Niu, C. Paus, D. Rankin, C. Roland, G. Roland, Z. Shi (1), G. S. F. Stephans (10, K. Sumorok, K. Tatar (1), D. Velicanu, J. Wang, T. W. Wang, Z. Wang, B. Wyslouch (1)

University of Minnesota, Minneapolis, USA

R. M. Chatterjee, A. Evans (1), S. Guts ${ }^{\dagger}$, P. Hansen, J. Hiltbrand, Sh. Jain (1), M. Krohn, Y. Kubota, Z. Lesko, J. Mans (1), M. Revering, R. Rusack, R. Saradhy, N. Schroeder, N. Strobbe (1), M. A. Wadud 


\section{University of Mississippi, Oxford, USA}

J. G. Acosta, S. Oliveros

\section{University of Nebraska-Lincoln, Lincoln, USA}

K. Bloom (1), S. Chauhan (1), D. R. Claes, C. Fangmeier, L. Finco (1), F. Golf(D) J. R. González Fernández, I. Kravchenko (1), J. E. Siado, G. R. Snow ${ }^{\dagger}$, B. Stieger, W. Tabb, F. Yan

\section{State University of New York at Buffalo, Buffalo, USA}

G. Agarwal, C. Harrington, L. Hay, I. Iashvili (1), A. Kharchilava, C. McLean (1), D. Nguyen, A. Parker, J. Pekkanen, S. Rappoccio (1), B. Roozbahani

\section{Northeastern University, Boston, USA}

G. Alverson (1), E. Barberis, C. Freer, Y. Haddad (10, A. Hortiangtham, G. Madigan, B. Marzocchi (1), D. M. Morse (1), V. Nguyen, T. Orimoto, L. Skinnari (1), A. Tishelman-Charny, T. Wamorkar, B. Wang, A. Wisecarver, D. Wood (1)

\section{Northwestern University, Evanston, USA}

S. Bhattacharya, J. Bueghly, Z. Chen, A. Gilbert (1), T. Gunter, K. A. Hahn, N. Odell, M. H. Schmitt (1), K. Sung, M. Velasco

\section{University of Notre Dame, Notre Dame, USA}

R. Bucci, N. Dev (D), R. Goldouzian, M. Hildreth, K. Hurtado Anampa (1), C. Jessop, D. J. Karmgard, K. Lannon, W. Li, N. Loukas (1), N. Marinelli, I. Mcalister, F. Meng, K. Mohrman, Y. Musienko ${ }^{46}$, R. Ruchti, P. Siddireddy, S. Taroni (1), M. Wayne, A. Wightman, M. Wolf(1), L. Zygala

The Ohio State University, Columbus, USA

J. Alimena (1), B. Bylsma, B. Cardwell, L. S. Durkin, B. Francis, C. Hill (1), A. Lefeld, B. L. Winer, B. R. Yates (1)

\section{Princeton University, Princeton, USA}

G. Dezoort, P. Elmer (1D, B. Greenberg, N. Haubrich, S. Higginbotham, A. Kalogeropoulos (D), G. Kopp, S. Kwan, D. Lange,

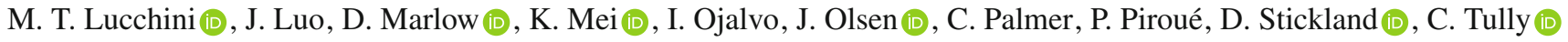

\section{University of Puerto Rico, Mayaguez, USA}

\section{S. Malik (1), S. Norberg}

\section{Purdue University, West Lafayette, USA}

V. E. Barnes (1), R. Chawla, S. Das, L. Gutay, M. Jones, A. W. Jung (), B. Mahakud, G. Negro, N. Neumeister (1),

C. C. Peng, S. Piperov (1D, H. Qiu, J. F. Schulte (i), N. Trevisani (1), F. Wang (1D), R. Xiao, W. Xie

\section{Purdue University Northwest, Hammond, USA}

T. Cheng, J. Dolen, N. Parashar, M. Stojanovic

\section{Rice University, Houston, USA}

A. Baty (1), S. Dildick, K. M. Ecklund (1), S. Freed, F. J. M. Geurts (1), M. Kilpatrick, A. Kumar, W. Li, B. P. Padley (1),

R. Redjimi, J. Roberts ${ }^{\dagger}$, J. Rorie, W. Shi (1), A. G. Stahl Leiton (1), A. Zhang

\section{University of Rochester, Rochester, USA}

A. Bodek (1), P. de Barbaro, R. Demina, J. L. Dulemba, C. Fallon, T. Ferbel, M. Galanti, A. Garcia-Bellido, O. Hindrichs, A. Khukhunaishvili, E. Ranken, R. Taus

\section{Rutgers, The State University of New Jersey, Piscataway, USA}

B. Chiarito, J. P. Chou (1), A. Gandrakota, Y. Gershtein (1), E. Halkiadakis (1), A. Hart, M. Heindl (1), E. Hughes, S. Kaplan,

O. Karacheban (1) ${ }^{25}$, I. Laflotte, A. Lath (1), R. Montalvo, K. Nash, M. Osherson, S. Salur (1), S. Schnetzer, S. Somalwar (1),

R. Stone, S. A. Thayil, S. Thomas, H. Wang

\section{University of Tennessee, Knoxville, USA}

H. Acharya, A. G. Delannoy (1), S. Spanier

\section{Texas A\&M University, College Station, USA}

O. Bouhali (ํ) ${ }^{91}$, M. Dalchenko (D), A. Delgado, R. Eusebi, J. Gilmore, T. Huang, T. Kamon ${ }^{92}$, H. Kim, S. Luo, S. Malhotra, R. Mueller, D. Overton, L. Perniè (i), D. Rathjens (i), A. Safonov (i), J. Sturdy (1) 


\section{Texas Tech University, Lubbock, USA}

N. Akchurin, J. Damgov, V. Hegde, S. Kunori, K. Lamichhane, S. W. Lee (1), T. Mengke, S. Muthumuni, T. Peltola (1), S. Undleeb, I. Volobouev, Z. Wang, A. Whitbeck

\section{Vanderbilt University, Nashville, USA}

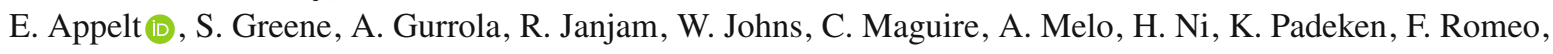
P. Sheldon (1), S. Tuo, J. Velkovska (1), M. Verweij (1)

\section{University of Virginia, Charlottesville, USA}

L. Ang, M. W. Arenton, B. Cox, G. Cummings, J. Hakala, R. Hirosky (ㅁ, M. Joyce, A. Ledovskoy, C. Neu (1),

B. Tannenwald (1), Y. Wang, E. Wolfe, F. Xia

\section{Wayne State University, Detroit, USA}

P. E. Karchin, N. Poudyal [1], P. Thapa

\section{University of Wisconsin-Madison, Madison, WI, USA}

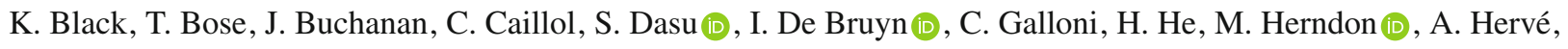
U. Hussain, A. Lanaro, A. Loeliger, R. Loveless, J. Madhusudanan Sreekala (10, A. Mallampalli, D. Pinna, T. Ruggles, A. Savin, V. Shang, V. Sharma (1), W. H. Smith (1), D. Teague, S. Trembath-reichert, W. Vetens

\section{$\dagger$ Deceased}

1: Vienna University of Technology, Vienna, Austria

2: Institute of Basic and Applied Sciences, Faculty of Engineering, Arab Academy for Science, Technology and Maritime Transport, Alexandria, Egypt, Alexandria, Egypt

3: Université Libre de Bruxelles, Bruxelles, Belgium

4: IRFU, CEA, Université Paris-Saclay, Gif-sur-Yvette, France

5: Universidade Estadual de Campinas, Campinas, Brazil

6: Federal University of Rio Grande do Sul, Porto Alegre, Brazil

7: UFMS, Nova Andradina, Brazil

8: Universidade Federal de Pelotas, Pelotas, Brazil

9: University of Chinese Academy of Sciences, Beijing, China

10: Institute for Theoretical and Experimental Physics named by A.I. Alikhanov of NRC 'Kurchatov Institute', Moscow, Russia

11: Joint Institute for Nuclear Research, Dubna, Russia

12: Cairo University, Cairo, Egypt

13: Suez University, Suez, Egypt

14: British University in Egypt, Cairo, Egypt

15: Zewail City of Science and Technology, Zewail, Egypt

16: Fayoum University, El-Fayoum, Egypt

17: Purdue University, West Lafayette, USA

18: Université de Haute Alsace, Mulhouse, France

19: Tbilisi State University, Tbilisi, Georgia

20: Erzincan Binali Yildirim University, Erzincan, Turkey

21: CERN, European Organization for Nuclear Research, Geneva, Switzerland

22: RWTH Aachen University, III. Physikalisches Institut A, Aachen, Germany

23: University of Hamburg, Hamburg, Germany

24: Department of Physics, Isfahan University of Technology, Isfahan, Iran, Isfahan, Iran

25: Brandenburg University of Technology, Cottbus, Germany

26: Skobeltsyn Institute of Nuclear Physics, Lomonosov Moscow State University, Moscow, Russia

27: Institute of Physics, University of Debrecen, Debrecen, Hungary, Debrecen, Hungary

28: Physics Department, Faculty of Science, Assiut University, Assiut, Egypt

29: MTA-ELTE Lendület CMS Particle and Nuclear Physics Group, Eötvös Loránd University, Budapest, Hungary, Budapest, Hungary

30: Institute of Nuclear Research ATOMKI, Debrecen, Hungary

31: IIT Bhubaneswar, Bhubaneswar, India, Bhubaneswar, India 
32: Institute of Physics, Bhubaneswar, India

33: G.H.G. Khalsa College, Punjab, India

34: Shoolini University, Solan, India

35: University of Hyderabad, Hyderabad, India

36: University of Visva-Bharati, Santiniketan, India

37: Indian Institute of Technology (IIT), Mumbai, India

38: Deutsches Elektronen-Synchrotron, Hamburg, Germany

39: Department of Physics, University of Science and Technology of Mazandaran, Behshahr, Iran

40: , INFN Sezione di Baria, Università di Barib, Politecnico di Bari, Bari, Italy

41: Italian National Agency for New Technologies, Energy and Sustainable Economic Development, Bologna, Italy

42: Centro Siciliano di Fisica Nucleare e di Struttura Della Materia, Catania, Italy

43: Riga Technical University, Riga, Latvia, Riga, Latvia

44: Consejo Nacional de Ciencia y Tecnología, Mexico City, Mexico

45: Warsaw University of Technology, Institute of Electronic Systems, Warsaw, Poland

46: Institute for Nuclear Research, Moscow, Russia

47: National Research Nuclear University 'Moscow Engineering Physics Institute' (MEPhI), Moscow, Russia

48: Institute of Nuclear Physics of the Uzbekistan Academy of Sciences, Tashkent, Uzbekistan

49: St. Petersburg State Polytechnical University, St. Petersburg, Russia

50: University of Florida, Gainesville, USA

51: Imperial College, London, UK

52: P.N. Lebedev Physical Institute, Moscow, Russia

53: California Institute of Technology, Pasadena, USA

54: Budker Institute of Nuclear Physics, Novosibirsk, Russia

55: Faculty of Physics, University of Belgrade, Belgrade, Serbia

56: Università degli Studi di Siena, Siena, Italy

57: Trincomalee Campus, Eastern University, Sri Lanka, Nilaveli, Sri Lanka

58: INFN Sezione di Pavia, Università di Pavia, Pavia, Italy

59: National and Kapodistrian University of Athens, Athens, Greece

60: Universität Zürich, Zurich, Switzerland

61: Stefan Meyer Institute for Subatomic Physics, Vienna; Austria, Vienna, Austria

62: Laboratoire d'Annecy-le-Vieux de Physique des Particules, IN2P3-CNRS, Annecy-le-Vieux, France

63: Şırnak University, Sirnak, Turkey

64: Department of Physics, Tsinghua University, Beijing; China, Beijing, China

65: Near East University, Research Center of Experimental Health Science, Nicosia, Turkey

66: Beykent University, Istanbul; Turkey, Istanbul, Turkey

67: Istanbul Aydin University, Application and Research Center for Advanced Studies (App. \& Res. Cent. for Advanced Studies), Istanbul, Turkey

68: Mersin University, Mersin, Turkey

69: Piri Reis University, Istanbul, Turkey

70: Adiyaman University, Adiyaman, Turkey

71: Ozyegin University, Istanbul, Turkey

72: Izmir Institute of Technology, Izmir, Turkey

73: Necmettin Erbakan University, Konya, Turkey

74: Bozok Universitetesi Rektörlügü, Yozgat; Turkey, Yozgat, Turkey

75: Marmara University, Istanbul, Turkey

76: Milli Savunma University, Istanbul, Turkey

77: Kafkas University, Kars, Turkey

78: Istanbul Bilgi University, Istanbul, Turkey

79: Hacettepe University, Ankara, Turkey

80: School of Physics and Astronomy, University of Southampton, Southampton, UK

81: IPPP Durham University, Durham, UK

82: Monash University, Faculty of Science, Clayton, Australia

83: Bethel University, St. Paul, Minneapolis; USA, St. Paul, USA 
84: Karamanoğlu Mehmetbey University, Karaman, Turkey

85: Ain Shams University, Cairo, Egypt

86: Bingol University, Bingol, Turkey

87: Georgian Technical University, Tbilisi, Georgia

88: Sinop University, Sinop, Turkey

89: Mimar Sinan University, Istanbul, Istanbul, Turkey

90: Nanjing Normal University Department of Physics, Nanjing, China

91: Texas A\&M University at Qatar, Doha, Qatar

92: Kyungpook National University, Daegu, Korea, Daegu, Korea 